\title{
Recent Progress of Two-Dimensional Thermoelectric Materials
}

Cite as

Nano-Micro Lett.

(2020) $12: 36$

Received: 26 October 2019

Accepted: 24 December 2019

Published online: 23 January 2020

(C) The Author(s) 2020

\author{
Delong $\mathrm{Li}^{1}$, Youning Gong ${ }^{1}$, Yuexing Chen ${ }^{2}$, Jiamei Lin ${ }^{1}$, Qasim Khan ${ }^{3}$, \\ Yupeng Zhang ${ }^{1 凶}, \mathrm{Yu} \mathrm{Li}^{4 凶}$, Han Zhang ${ }^{1 凶}$, Heping $\mathrm{Xie}^{5}$ \\ $\square$ Yupeng Zhang, ypzhang@szu.edu.cn; Yu Li, liyu@szu.edu.cn; Han Zhang, hzhang@szu.edu.cn \\ 1 Collaborative Innovation Centre for Optoelectronic Science \& Technology, and Key Laboratory \\ of Optoelectronic Devices and Systems of Ministry of Education and Guangdong Province, Institute \\ of Microscale Optoelectronics, College of Chemistry and Environmental Engineering, Shenzhen University, \\ Shenzhen 518060, Guangdong, People's Republic of China \\ 2 Shenzhen Key Laboratory of Advanced Thin Films and Applications, College of Physics and Optoelectronic \\ Engineering, Shenzhen University, Shenzhen 518060, Guangdong, People's Republic of China \\ 3 Department of Mechanical and Mechatronics Engineering, University of Waterloo, Waterloo, ON, Canada \\ 4 Shenzhen Key Laboratory of Special Functional Materials, Shenzhen Engineering Laboratory for Advanced \\ Technology of Ceramics, Guangdong Research Center for Interfacial Engineering of Functional Materials, \\ College of Materials Science and Engineering, Shenzhen University, Shenzhen 518060, Guangdong, \\ People's Republic of China \\ 5 Shenzhen Clean Energy Research Institute, Shenzhen University, Shenzhen 518060, Guangdong, \\ People's Republic of China
}

\section{HIGHLIGHTS}

- A comprehensive review on the recent development of two-dimensional (2D) nanomaterials for bulk or thin-film thermoelectric materials, as well as composite filler, has been extensively presented.

- Development of micro-device platform and its application to study the inherent thermoelectric properties of individual single- and few-layer 2D nanomaterials.

\begin{abstract}
Thermoelectric generators have attracted a wide research interest owing to their ability to directly convert heat into electrical power. Moreover, the thermoelectric properties of traditional inorganic and organic materials have been significantly improved over the past few decades. Among these compounds, layered two-dimensional (2D) materials, such as graphene, black phosphorus, transition metal dichalcogenides, IVA-VIA compounds, and MXenes, have generated a large research attention as a group of potentially high-performance thermoelectric materials. Due to their unique electronic, mechanical, thermal, and optoelectronic properties, thermoelectric devices based on such materials can be applied in a variety of applications. Herein, a comprehensive review on the development of 2D materials for thermoelectric applications, as well as theoretical simulations and experimental preparation, is presented. In addition, nanodevice and new applications of $2 \mathrm{D}$ thermoelectric materials are also introduced. At last, current challenges are discussed and several prospects in this field are proposed.
\end{abstract}

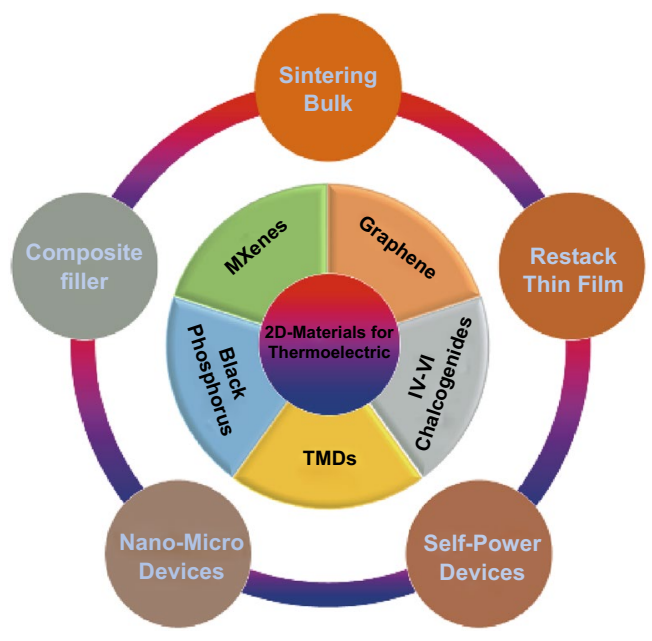

KEYWORDS Two-dimensional thermoelectric materials; Black phosphorus analogue; Tin selenide; Transition metal dichalcogenides; Photothermoelectric effect 


\section{Introduction}

Due to the increasing demand in high-efficiency clean energy, it is essential to develop renewable energy devices to resolve the energy issues and avoid further environmental deterioration $[1,2]$. In the past several decades, thermoelectric device, solar cells, wind-driven generators and fuel cells have attracted a large attention and shown a reliable quality in power generation. Among these kinds of energy conversion technologies, thermoelectric devices show a high potential in their application in many areas including power generators, cooling devices, and sensors [3-6]. Thermoelectric materials can directly convert thermal energy (such as waste heat and solar energy) into electrical energy; this property makes them important compounds for the development of sustainable energy efficient technologies [7-9]. When compared with other energy conversion devices, thermoelectric device show unique advantages including stability, long service life, and noiseless [10-12]. The thermoelectric performance of the materials at a certain temperature is evaluated by the dimensionless figure of merit (ZT), ZT $=\sigma S^{2} T / \kappa$. In this expression, $\sigma, T$, and $\kappa$ correspond to the electrical conductivity, the Seebeck coefficient, and the absolute temperature, respectively. Moreover, $\kappa_{\mathrm{L}}$ refers to the lattice thermal conductivity $\kappa_{(\mathrm{L})}$ and $\kappa_{(\mathrm{e})}$ to the electronic thermal conductivity $[13,14]$. These parameters are strongly coupled and dependent on the material's band and crystal structure. Due to the complex inter-relation among these three parameters, it is almost impossible to optimize them independently [15]. However, several records for the highest ZT values have been continuously broken in the last few years due to the development of novel materials, new processing techniques, and new concept/mechanisms [16-20].

Since the initial discovery of graphene in 2004, the research interest in these materials has been growing explosively in the last decades [21, 22]. With the development of novel theoretical simulation methods and materials synthesis technics, a variety of 2D materials have been theoretically predicted and successfully fabricated. Typical 2D materials are graphene, black phosphorus (BP), transition metal dichalcogenides (TMDCs) (e.g., $\mathrm{MoS}_{2}, \mathrm{WS}_{2}, \mathrm{MoSe}_{2}$, and $\mathrm{MoTe}_{2}$ ), Group IVA-VA compounds (e.g., SnSe, GeSe, and $\mathrm{SnS}$ ), nitrides (e.g., boron nitride), MXenes (e.g., $\mathrm{Ti}_{3} \mathrm{C}_{2}$, and
$\mathrm{Ti}_{4} \mathrm{~N}_{3}$ ), and Xenes (e.g., black phosphorene, arsenene, bismuthine, and antimonene) [21, 23-34] These 2D materials exhibit different allotropes with outstanding electronic and optical properties. For this reason, they have been widely used in electronics, optoelectronics, topological spintronic, bio-application energy storage (e.g., battery and supercapacitors) and energy conversion devices (e.g., thermoelectric and solar cells) [35-38].

Due to their outstanding advantages in electronic and mechanical properties, 2D materials with a layered structure have attracted a considerable attention as efficient thermoelectric materials $[8,39]$. In the past several decades, the thermoelectric performance of a series of 2D materials, such as $\mathrm{SnSe}, \mathrm{Bi}_{2} \mathrm{Te}_{3}$, and $\mathrm{MoS}_{2}$, has been theoretically predicted and the samples have been experimentally fabricated $[33,34,40]$. These 2D materials exhibit fascinating properties exhibit such as a large potential when they are used in the fabrication of high-performance thermoelectric devices.

Currently, although interesting research results in both theoretical predictions and experimental analyses have been achieved, a comprehensive review about 2D thermoelectric materials is still missing. To promote their development, it is pivotal to focus on the research progress in this field. In this review, the theoretical and experimental advances in the 2D thermoelectric materials field are summarized. Initially, their unique electrical and thermal properties are illustrated. Then, the application of 2D nanomaterials to fabricate bulk thermoelectric compounds, thin-film thermoelectric materials, and composite fillers is discussed in detail. Moreover, the thermoelectric properties of single- or multilayer 2D materials studied by using nano-micro-devices are introduced. Finally, thermoelectric compounds combined with photodetection devices are discussed. A perspective and an outlook on 2D thermoelectric materials conclude this review.

\section{Properties of 2D Thermoelectric Materials}

\subsection{Graphene}

As a typical 2D material, graphene has become a popular topic in scientific research due to its distinctive physical and chemical properties since it can be exfoliated from bulk graphite [41, 42]. Due to its unique electrical, optical, 
catalytic, and mechanical features, graphene has attracted a broad attention in recent years in many fields. For instance, graphene shows an ultrahigh electrical conductivity $\left(10^{6} \mathrm{~S} \mathrm{~cm}^{-1}\right)$ at room temperature due to its high electron mobility [43]. Its maximum Seebeck coefficient value was reported to be about $80 \mathrm{mV} \mathrm{K}^{-1}[27,44,45]$. The thermal conductivity of graphene is in the $4840-5300 \mathrm{~W} \mathrm{mK}^{-1}$ range at room temperature [46]. A large theoretical and experimental effort has been done to study the thermoelectric performance of graphene [44, 45, 47-69].

Nam et al. investigated the in-plane thermoelectric properties of bilayer graphene by using a micro-device, and their results were simulated by using the Mott formula with a hyperbolic dispersion relation [49]. The gate-voltage dependence of the thermoelectric properties of bilayer graphene has been carried out for various temperatures and charge-carrier densities. Figure 1a-d shows the measured Seebeck coefficient of bilayer graphene, which follows the semiclassical Mott formula at low temperature $(T=30 \mathrm{~K}$ and $T=50 \mathrm{~K})$. However, at high temperature ( $T=140 \mathrm{~K}$ and $T=250 \mathrm{~K}$ ), the Seebeck coefficient measured by using the micro-device reveals a deviation from the simulated Seebeck coefficient near the charge neutrality point, and this deviation increases with the temperature [49]. Figure 1e, f shows the simulation of the electron and phonon transport through an edge-disordered zigzag graphene layer [50]. The simulation results on the graphene nanoribbons with different sample lengths and number of zigzag chains $(\mathrm{Nz})$ were studied. Sevincli et al. found that the edge disorder in zigzag graphene nanoribbons (ZGNRs) can significantly reduce the phonon thermal conductance, whereas the electronic conduction remains almost intact at the first conductance plateau [50]. Sevincli predicted that the edge disorder suppresses the thermal conductivity by few orders of magnitude in the zigzag edges graphene nanoribbons. Moreover, it gives rise to a ZT value of 4 at $300 \mathrm{~K}$ [50].

These results indicate that graphene can be optimized to achieve high-performance thermoelectric properties. However, for practical applications, both the Seebeck coefficient and the power factor of graphene should be improved, whereas its thermal conductivity should be decreased. To achieve large ZT values in graphene-based thermoelectric materials, two major barriers need to be overcome: (1) the graphene thermal conductivity is too high, and (2) the Seebeck coefficient is too small due to its gapless band structure. Although graphene exhibits advantage as a
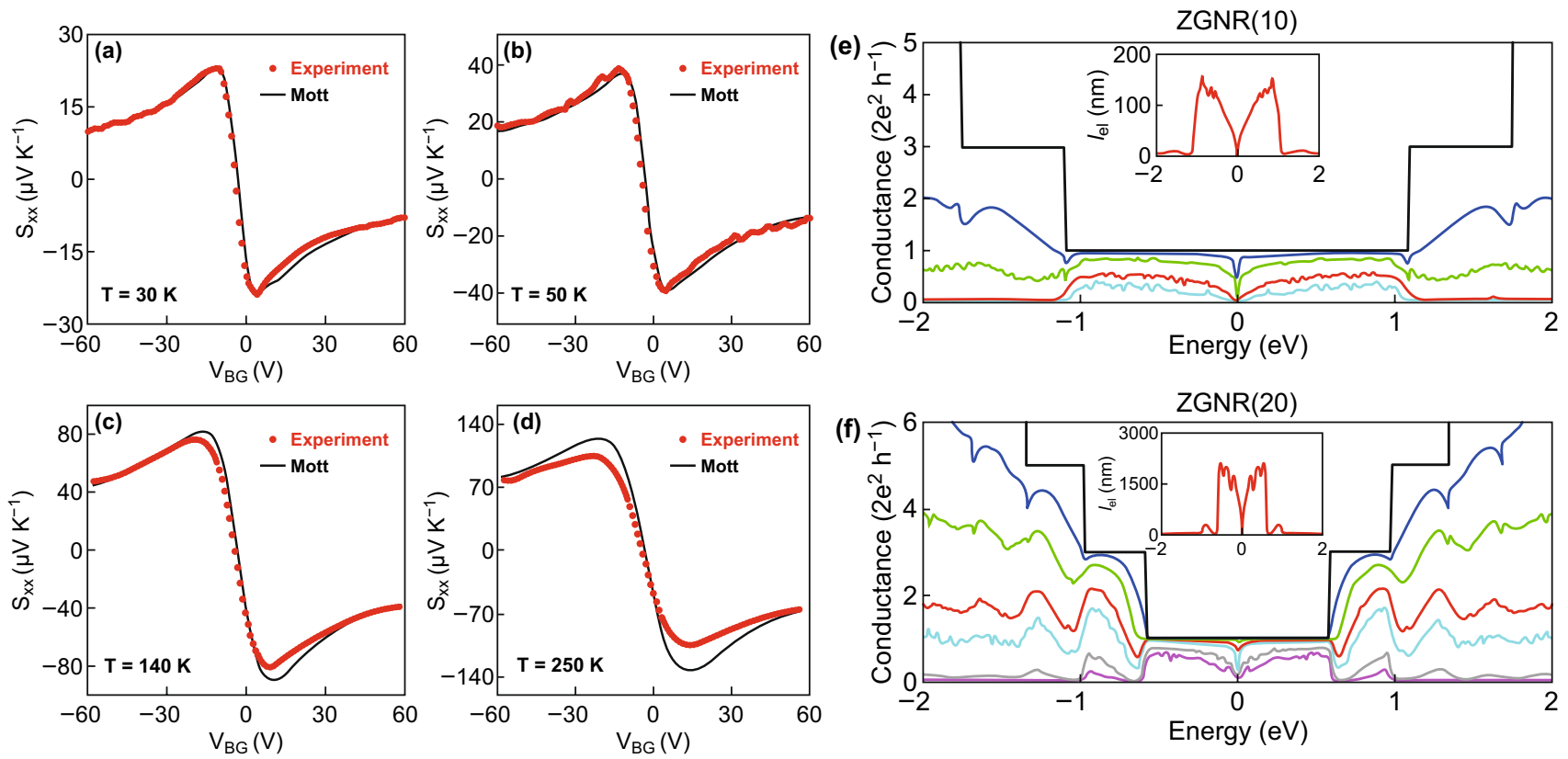

Fig. 1 The in-plane Seebeck coefficient of bilayer graphene as a function of the backgate voltage $\left(V_{\mathrm{BG}}\right)$ at different temperatures: a $T=30 \mathrm{~K}$, b $T=50 \mathrm{~K}, \mathbf{c} T=140 \mathrm{~K}$ and, d $T=250 \mathrm{~K}$. Reproduced with permission from Ref. [49]. Copyright 2010, American Physical Society. Electron transport through the edge-disordered zigzag edges graphene nanoribbons: sample lengths $\mathbf{e} \mathrm{Nz}=10$ and $\mathbf{f} \mathrm{Nz}=20$. Reproduced with permission from Ref. [50]. Copyright 2010, American Physical Society 
high-performance thermoelectric material, more efforts in simulations and experiments need to be done to optimize its thermoelectric performance.

\subsection{TMDC}

As a novel class of layered materials, 2D TMDCs, such as $\mathrm{MX}_{2}(\mathrm{M}=\mathrm{Mo}, \mathrm{W}, \mathrm{Ti}$, and $\mathrm{X}=\mathrm{S}, \mathrm{Se}, \mathrm{Te})$, have attracted a large attention in last decades due to their semiconducting characteristics, outstanding chemical stability, and mechanical and physical properties. Moreover, they have been widely studied in various fields including optoelectronic, energy harvests and conversion, and cancer therapy. These types of material have been widely studied in many fields, such as photodetector, thermoelectric, and gas-sensing applications. Their relatively high electrical conductivity and relatively low thermal conductivity make the TMDCs emerge as promising materials for high-performance thermoelectric devices, especially for the fabrication of wearable heating/cooling devices and power generators.

The thermoelectric properties of 2D TMDCs with different thickness have been investigated both experimentally and theoretically. The most common TMDCs, such as $\mathrm{MoS}_{2}, \mathrm{MoSe}_{2}, \mathrm{WS}_{2}$, and $\mathrm{WSe}_{2}$, exhibit very similar electronic properties. For instance, the $\mathrm{MoS}_{2}$ monolayer is a direct semiconductor with a band gap of $1.9 \mathrm{eV}$, whereas bulk $\mathrm{MoS}_{2}$ is an indirect semiconductor with a band gap of $1.2 \mathrm{eV}$ [70]. The band structures of the 2D TMDCs share a similar transition from the direct band gap to the indirect band gap, as the atomic layer increases from a monolayer to a bilayer [8].

As previously mentioned, the ZT value depends on three inherent physical quantities: the thermal conductivity, the electrical conductivity, and the Seebeck coefficient. These quantities have been widely studied in the case of 2D materials. Owing to their characteristic quasi-two- dimensional crystal structures, TMDCs exhibit high Seebeck coefficient [71-73]. By considering $\mathrm{MoS}_{2}$ as a reference compound, a brief introduction about the thermoelectric properties of 2D TMDCs is presented below. As shown in Fig. $2 \mathrm{a}-\mathrm{c}$, the thermoelectric properties of $\mathrm{MoS}_{2}$ multilayers at $300 \mathrm{~K}$ are calculated along the in-plane direction. According to the calculation results, the Seebeck coefficients are not affected so much by the change of the layer numbers. However, the electric conductivity shows clear changes depending on the layer thickness, as shown in Fig. 2b. The significantly increased electrical conductivity as well as the $P F / \tau$ is mainly caused by the valley degeneracy at the valence band edge [73]. The ZT values of TMDCs $\left(\mathrm{MoSe}_{2}, \mathrm{WS}_{2}\right.$, and $\mathrm{WSe}_{2}$ ) along the in-plane direction have been predicted. As shown in Fig. 2d-f, the few layers' structure shows large increase in the values of their ZT compared to those of the bulk. Besides the theory prediction, the thermoelectric properties of 2D TMDCs have also been measured and the experimental results have proven that their thermoelectric properties can be enhanced by applying external electric field and pressure. Buscema et al. [71] reported a large Seebeck coefficient with a large tunability between $-4 \times 10^{2}$ and $-1 \times 10^{5} \mu \mathrm{V} \mathrm{K} \mathrm{K}^{-1}$ for the $\mathrm{MoS}_{2}$ monolayer in the presence of an external electric field. However, in a highly positive gate range, the Seebeck coefficient varies from $-2 \times 10^{2}$ to $-1.5 \times 10^{3} \mu \mathrm{V} \mathrm{K} \mathrm{K}^{-1}$ and in a highly negative gate range, it varies from $-3 \times 10^{4}$ to $-3 \times 10^{5} \mu \mathrm{V} \mathrm{K}^{-1}$, showing an increases of about two orders of magnitude. $\mathrm{Wu}$ et al. observed a large Seebeck coefficient of $30 \mathrm{mV} \mathrm{K}^{-1}$ by adjusting the backgate voltage [74]. The thermoelectric properties of an exfoliated $2 \mathrm{D} \mathrm{MoS}$ flake with different thickness were studied by Hippalgaonkar. Due to the high electron concentration of $n=1.06 \times 10^{13} \mathrm{~cm}^{-2}$, the bilayer $\mathrm{MoS}_{2}$ exhibits the highest power factor $\left(8.5 \mathrm{~mW} \mathrm{mK}^{-2}\right)$ as the gate voltage is $104 \mathrm{~V}$ [72]. Due to the high electron concentration of $n=1.06 \times 10^{13} \mathrm{~cm}^{-2}$, the $\mathrm{MoS}_{2}$ bilayer exhibits the highest power factor $\left(8.5 \mathrm{~mW} \mathrm{mK}^{-2}\right)$ as the gate voltage reaches $104 \mathrm{~V}$.

The 2D TMDCs exhibit a relatively lower thermal conductivity when compared to graphene, which make them a promising material for various applications in thermoelectric devices. The low thermal conductivity of the 2D TMDCs has been widely studied in experimental and theoretical works [75-89]. For instance, Sahoo et al. measured the thermal conductivity of a suspended fewlayer $\mathrm{MoS}_{2}$ film by using a laser-power-dependent Raman scattering experiment method. As a result, the thermal conductivity at room temperature for few-layer $\mathrm{MoS}_{2}$ was found to be $52 \mathrm{~W} \mathrm{mK}^{-1}$ [80]. Yan et al. [79] carefully measured and analyzed the temperature-dependent Raman spectra of the exfoliated $\mathrm{MoS}_{2}$ monolayer at room temperature, and the measured thermal conductivity is $34.5 \mathrm{~W} \mathrm{mK}^{-1}$. Taube et al. measured the temperaturedependent thermal conductivity of the $\mathrm{MoS}_{2}$ monolayer on a $\mathrm{SiO}_{2} / \mathrm{Si}$ substrate via Raman spectroscopy. The 

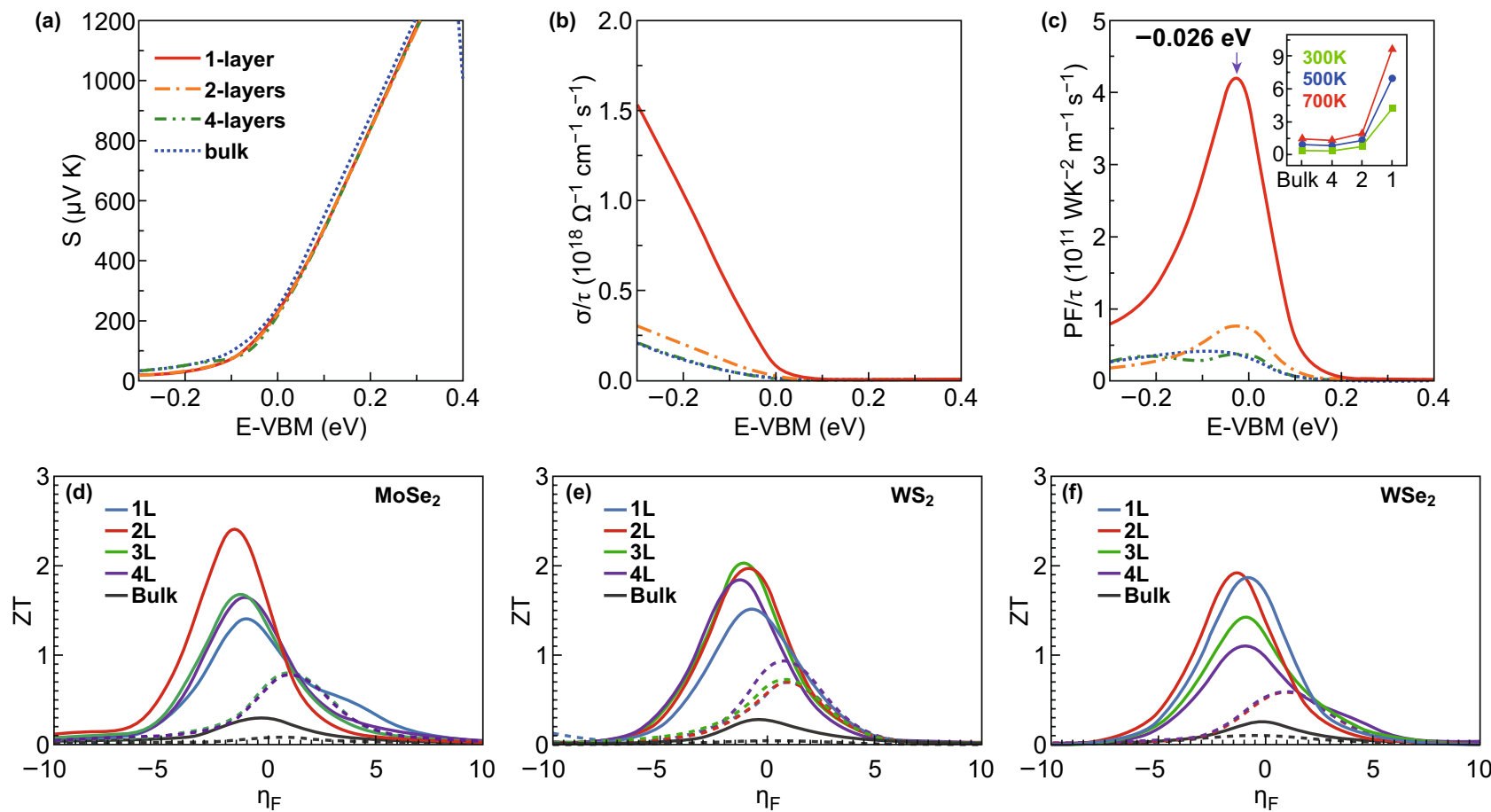

Fig. 2 a In-plane Seebeck coefficient, b electrical conductivity, and c power factor of p-type $\mathrm{MoS}_{2}$ at $300 \mathrm{~K}$ depending on their thickness and chemical potential. Reproduced with permission from Ref. [73]. Copyright 2016, American Physical Society. ZT at $300 \mathrm{~K}_{\text {for }} \mathbf{d ~ M o S e} \mathrm{M}_{2}$, e WS ${ }_{2}$, and $\mathbf{f} \mathrm{WSe}_{2}$ for bulk (black), 1 layer (blue), 2 layers (red), 3 layers (green), and 4 layers (purple) structures as a function of the reduced Fermi energy, $\eta_{\mathrm{F}}$. The $n$-type ZT is plotted with a solid line and $p$-type ZT with a broken line. Reproduced with permission from Ref. [90] Copyright 2014, AIP Publishing. (Color figure online)

results show that the thermal conductivity decreases from 62.2 to $7.45 \mathrm{~W} \mathrm{mK}^{-1}$ as the temperature increases from 300 to $450 \mathrm{~K}[78]$.

In addition, the wide tunability of inherent thermoelectric parameters of a $\mathrm{MoS}_{2}$ monolayer or a few layers makes this material an ideal candidate for applications as a conventional thermoelectric generator or a cooler. Although the calculation predictions show that the TMDCs should exhibit an outstanding thermoelectric performance, these results have not been proven yet. The measured Seebeck coefficient is much lower than the calculations suggest data, and the thermal conductivity is much higher than in the case of conventional thermoelectric materials but much lower than graphene.

\subsection{Group IVA-VIA Compounds}

As the most efficient kinds of thermoelectric materials, the compounds that belong to group IVA-VIA have became the hot research field of thermoelectric science during the past decades. These materials, such as $\mathrm{Sn}(\mathrm{S}, \mathrm{Se}, \mathrm{T}(\mathrm{e}), \mathrm{Ge}(\mathrm{S}, \mathrm{Se}$, $\mathrm{Te}), \mathrm{Pb}(\mathrm{S}, \mathrm{Se}, \mathrm{Te}), \mathrm{Sn}(\mathrm{Se}, \mathrm{S})_{2}$, and their alloys, have been widely studied in the thermoelectric field. Moreover, they have been used to fabricate high-performance thermoelectric power generators, thermoelectric sensors, and cooling devices [91, 92]. Among them, $\mathrm{Sn}(\mathrm{S}, \mathrm{Se})$ and $\mathrm{Ge}(\mathrm{S}, \mathrm{Se})$, which belong to the space group Pnma, exhibit a typical layered 2D structure feature along the $c$ direction. The reduction in the dimensionality of these materials has been proved as one of the most efficient methods to enhance their ZT values since the Seebeck coefficient increases due to the increased density of the states near the Fermi level [93, 94]. In 1993, by carefully studying the properties and the structure of a low-dimensional $\mathrm{Bi}_{2} \mathrm{Te}_{3}$ material, a theory to explain the relation between its structure and its thermoelectric properties was presented by Dresselhaus and Hicks [95]. According to their results, the numerous grain boundary and interfaces in the material would generate a strong phonon scattering, which leads to a reduction in its thermal conductivity. Because of this reduced thermal conductivity and almost 
unchanged electrical properties, an enhanced $\mathrm{ZT}$ value could be obtained in the case of such low-dimensional materials [95]. Several years later, Sofo and Mahan [96] proposed a modified theory. They suggested that the well-quantum mixing and the changing density of state from a $2 \mathrm{D}$ into a $3 \mathrm{D}$ lead to a higher thermoelectric performance. Subsequently, by converting them into the well-quantum, the highest $\mathrm{ZT}$ value of bulk $\mathrm{Bi}_{2} \mathrm{Te}_{3}$ was improved 13 times. This result confirms the validity of the theory that the reduction in the dimensionality is helpful to enhance the energy conversion efficiency of these materials [97]. Fei and Cheng [98, 99] reported a bismuth monolayer showing very promising thermoelectric properties.

As a typical compound belonging to the group IVA-VIA, tin selenide ( $\mathrm{SnSe}$ ) has been widely studied due to its outstanding electronic and photonic properties. SnSe exhibits potential applications in many fields such as in energy storage and conversion devices and novel optoelectronic devices [92, 100-102] SnSe exhibits a layered orthorhombic structure and belongs to the Pnma space group at room temperature. Its structure can be derived from a three-dimensional distortion of a rock-salt structure. Normally, the $\mathrm{Sn}$ and Se atoms are arranged in double layers with two planes consisting of zigzag $\mathrm{Sn}-\mathrm{Se}$ chains along the a-axis.

As a thermoelectric material, the thermoelectric properties of SnSe are limited by its poor electrical conductivity at room temperature. However, Zhao et al. reported a remarkably high ZT value of 2.6 (at $923 \mathrm{~K}$ ) and of 2.2 (at $773 \mathrm{~K}$ ) in $p$-type and $n$-type single-crystal SnSe, respectively [33, 103]. These results triggered the researchers in performing further studies on the thermoelectric properties of $\mathrm{SnSe}$ and its alloys. According to Zhao's study, such excellent thermoelectric performances originate from the ultralow thermal conductivity when the SnSe transfers into a high-temperature phase at $T>973 \mathrm{~K}$. Inspired by the ultralow thermal conductivity and by the excellent electrical properties, SnSe and its alloys have attracted a significant attention in recent years [104]. Despite the excellent thermoelectric properties obtained for the SnSe single crystals, their complex crystal growth conditions and high production costs limit their practical applications [105]. Therefore, scientists focused on the development of high-performance polycrystalline SnSe. Via systematic optimization methods, such as texturing, doping, and alloying, a significant enhancement of the ZT value of polycrystalline SnSe materials was achieved during the last several years [106-108]. Recently, the thermoelectric properties of polycrystalline SnSe have been improved continuously and their ZT value have been improved from 0.5 to nearly 1.7 via optimization of the carrier concentration [109, 110]. The thermoelectric performance of polycrystalline SnSe can further improved since its ZT value is still much lower than that of a single-crystal SnSe. For polycrystalline $\mathrm{SnSe}$, the thermal conductivity remains higher than its theoretical calculation value and its electrical conductivity is lower than a single-crystal $\mathrm{SnSe}$ due to the existing grain boundaries [111]. Achieving an ultrahigh ZT value for the polycrystalline comparable to its single-crystal counterpart is still a challenge, and more experimental and theoretical work needs to be done in the next years.

\subsection{Black Phosphorus}

A monolayer phosphorene and a few-layer phosphorene have been successfully exfoliated from bulk BP in 2014 and have attracted a tremendous research interest in the past few years $[28,112,113]$. As a novel elementary 2D material, the few-layer BP has gained tremendous attention in theoretical and experimental investigations [114-119]. Due to its unique crystal structure and in-plane anisotropic properties, BP has been widely studied in various fields including in the development of photodetectors [116, 120, 121], cancer therapies [115, 122, 123], supercapacitors [124], field-effect transistors (FETs) [125, 126], batteries [127, 128], and thermoelectric devices [39, 98, 129-133].

Moreover, BP has also been reported as a prospective materials for the production of thermoelectric devices due to its large Seebeck coefficient $\left(335 \mu \mathrm{V} \mathrm{K} \mathrm{K}^{-1}\right.$ at room temperature) [130], high carrier mobility $\left(1000 \mathrm{~cm}^{2}\right.$ $(\mathrm{Vs})^{-1}$ at room temperature) [28], and moderate band gap $(0.3-2.0 \mathrm{eV})[28,112,130]$. According to the experimental results, bulk BP displays a high Seebeck coefficient of $335 \mu \mathrm{V} \mathrm{K}^{-1}$ and the few-layer BP show an increased Seebeck coefficient up to $510 \mu \mathrm{V} \mathrm{K}^{-1}$ [130, 134]. Due to its puckered structure, the electrical conductivity, the Seebeck coefficient, and the thermal conductivity of BP exhibit a strongly in-plane anisotropic behavior. This characteristic has been proved theoretically and experimentally in various kinds of BP materials including few-layer BP, thin-film BP, and bulk BP [135-140]. Recently, a number of theoretical simulation results have been published and predicted that the few-layer BP is an appealing materials 
with outstanding thermoelectric properties. As shown in Fig. 3a, b, the Seebeck coefficient and the electrical conductivity were calculated by using the first-principles calculations and the Boltzmann transport theory. These results are presented as a function of the carrier concentration at $300 \mathrm{~K}$ [132]. As predicted, a peak ZT value of 1.1 was obtained with an electron concentration of $1.5 \times 10^{20} \mathrm{~cm}^{-3}$. The electrical conductivity of the fewlayer BP along the zigzag direction is much lower than along the armchair direction. The Seebeck coefficient almost maintains an identical level along all the directions. The thermal conductivities of the few-layer BP with different thicknesses are shown in Fig. 3c, d. It can be concluded that the thermal conductivity along the zigzag direction is higher than that along the armchair direction. Moreover, the ZT value can be further enhanced by doping the few-layer BP with a proper element. Zhang et al. [132]
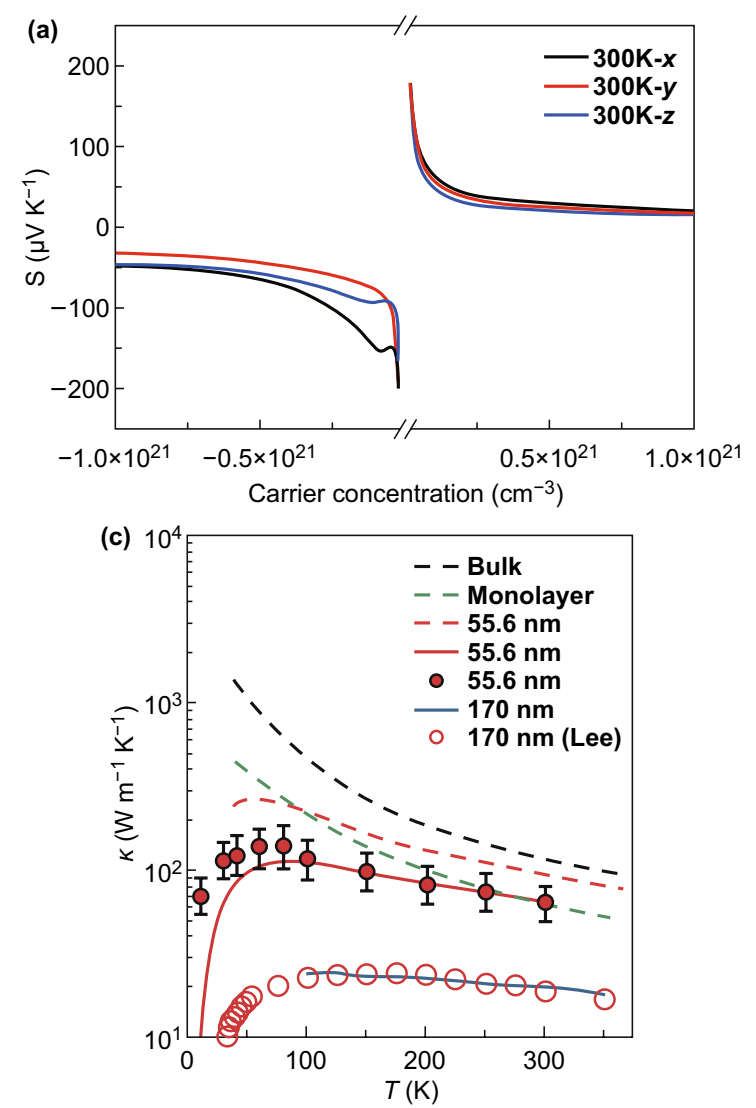

investigated the thermoelectric properties of the Sb-doped few-layer BP, and their calculation indicates that the highest $\mathrm{ZT}$ value measures almost 6.0 for $\mathrm{P}_{0.75} \mathrm{Sb}_{0.25}$. As shown in Fig. 3, the anisotropy of the electrical conductivity corresponds to the opposite of its thermal conductivity, which makes the study of BP for thermoelectric more challenging [39]. Although the theoretical results have predicted that $\mathrm{BP}$, the few-layer BP, and bulk BP exhibit a huge potential as excellent thermoelectric materials, only a few experimental results were reported. A larger experimental efforts need to be done to promote layered BP as a potential candidate for the production of thermoelectric devices.

\subsection{MXene}

As a newly developed group of 2D materials consisting of transition metal carbides and nitrides, MXenes exhibit
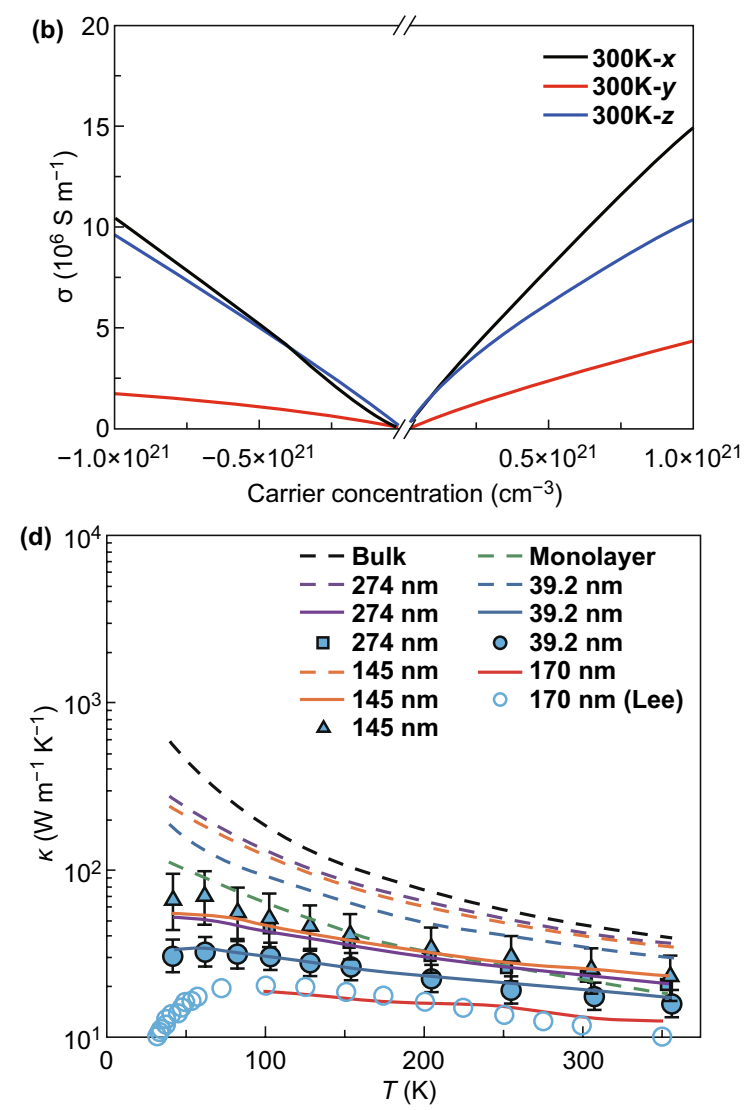

Fig. 3 a Calculated Seebeck coefficient and b electrical conductivity of a few-layer black phosphorus at $T=300 \mathrm{~K}$. Reproduced with permission from Ref. [132]. Copyright 2016, Royal Society of Chemistry. Measured thermal conductivity of black phosphorus depending on their thickness and temperature along different directions: $\mathbf{c}$ zigzag direction, $\mathbf{d}$ armchair direction. The dashed lines and the solid lines correspond to the calculation results for the defect-free black phosphorus and the black phosphorus with additional vacancy point defects, respectively. Reproduced with permission from Ref. [135]. Copyright 2017, Wiley-VCH 
either metallic or semiconducting properties depending on its surface functionalization [141]. MXenes can be prepared by exfoliating the MAX phases into 2D nanosheets via selective etching of "A" layers by using appropriate hydrofluoric acids [29, 141-145]. These materials are defined as $\mathrm{M}_{n+1} \mathrm{AX}_{n}$, where $n=1,2$, or 3, "M" corresponds to an early transition metal ( $\mathrm{Sc}, \mathrm{Ti}, \mathrm{V}, \mathrm{Cr}, \mathrm{Zr}, \mathrm{Nb}, \mathrm{Mo}, \mathrm{Hf}$, $\mathrm{Ta}$ ), "A" is an element from groups 13-16 in the periodic table ( $\mathrm{Al}, \mathrm{Si}, \mathrm{P}, \mathrm{S}, \mathrm{Ga}, \mathrm{Ge}, \mathrm{As}, \mathrm{In}, \mathrm{Sn}, \mathrm{Tl}, \mathrm{Pb}$ ), and " $X$ " is carbon and/or nitrogen. Due to its excellent thermal stability in air, MXene has been studied as a high-temperature thermoelectric material. Moreover, Kumar et al. have predicted that the functionalization of MXene by O, F, and $\mathrm{OH}$ groups can significantly affect its structural properties and electronic band structure, further enhancing its thermoelectric performance [146]. For instance, when $\mathrm{ScC}_{2}$ is functionalized with $\mathrm{C}, \mathrm{O}$, or $\mathrm{OH}$, its band gap measures 1.84 (indirect), 1.03 (indirect), and $0.44 \mathrm{eV}$ (indirect), respectively [146]. Such varied band gap structure leads to different thermoelectric properties of the materials. The calculation results show that the Seebeck coefficient of $\mathrm{Sc}_{2} \mathrm{C}(\mathrm{OH})_{2}$ measures only $372 \mu \mathrm{V} \mathrm{K}^{-1}$, while the Seebeck coefficient of $\mathrm{Sc}_{2} \mathrm{CO}_{2}$ and $\mathrm{Sc}_{2} \mathrm{CF}_{2}$ is above $1000 \mu \mathrm{V} \mathrm{K}$ at room temperature [146]. Furthermore, the calculation results show that these materials exhibit different thermal conductivity. The lattice thermal conductivity of $\mathrm{Sc}_{2} \mathrm{CO}_{2}$, $\mathrm{Sc}_{2} \mathrm{CF}_{2}$, and $\mathrm{Sc}_{2} \mathrm{C}(\mathrm{OH})_{2}$ is 59,36 , and $10 \mathrm{~W} \mathrm{mK}^{-1}$, respectively. By using the Boltzmann transport theory and the first-principles electronic structure calculations, Khazaei et al. [141] predicted the thermoelectric properties of more than 35 kinds of different functionalized MXene monolayers and multilayers of the type $\mathrm{M}_{2} \mathrm{C}$, where $\mathrm{M}=\mathrm{Sc}, \mathrm{Ti}, \mathrm{V}$, $\mathrm{Zr}, \mathrm{Nb}, \mathrm{Mo}, \mathrm{Hf}$, and $\mathrm{Ta}$, and $\mathrm{M}_{2} \mathrm{~N}, \mathrm{M}=\mathrm{Ti}, \mathrm{Zr}$, and Hf. The calculation results prove that the monolayer and multilayer $\mathrm{Mo}_{2} \mathrm{C}$ nanosheets exhibit a higher power factor than other functionalized MXenes samples. However, several positive results show that MXene can achieve a higher thermoelectric performance. According to the calculations, almost all the semiconducting MXene shows a Seebeck coefficient larger than $100 \mu \mathrm{V} \mathrm{K}^{-1}$ at $400 \mathrm{~K}$ and some of them (such as $\mathrm{Mo}_{2} \mathrm{CF}_{2}, \mathrm{Mo}_{2} \mathrm{C}(\mathrm{OH})_{2}$, and $\mathrm{Mo}_{2} \mathrm{CCl}_{2}$ ) exhibit a high electrical conductivity [141].

The outstanding thermoelectric performance of MXenes has also been proved by a series of experiments. Kim et al. fabricated two kinds of Mo-based MXene $\left(\mathrm{Mo}_{2} \mathrm{CT}_{x}\right.$, $\mathrm{Mo}_{2} \mathrm{TiC}_{2} \mathrm{~T}_{x}$, and $\mathrm{Mo}_{2} \mathrm{Ti}_{2} \mathrm{C}_{3} \mathrm{~T}_{x}$ ) flexible thin films and studied their thermoelectric properties [147]. For instance, the $\mathrm{Mo}_{2} \mathrm{TiC}_{2} \mathrm{~T}_{x}$ film exhibits the highest power factor (about $309 \mu \mathrm{W} \mathrm{mK}{ }^{-2}$ at $800 \mathrm{~K}$ ) among these samples. Although a high thermoelectric performance for MXene has been theoretically predicted [141, 146-148], more experimental results have to be performed.

\section{Thermoelectric Materials Based on 2D Materials}

Layered 2D materials have been widely employed as efficient thermoelectric materials in the last decades. In this section, the 2D layer structure materials based on the elements belonging to the groups IV-VI and on TMDCs are investigated. The previously reported results proved that bulk and monolayer materials exhibit extremely different thermoelectric properties [149]. In this section, the thermoelectric properties of bulk and thin films based on $2 \mathrm{D}$ materials are discussed.

\subsection{Bulk Thermoelectric Materials}

Due to the limitations in materials synthesis techniques, bulk thermoelectric materials are usually fabricated via a simple melting process or a ball milling process followed by a post-sintering process (such as spark plasma sintering or hot press process). Although the materials exhibit a layer-bylayer structure, the synthesis of these compounds via these methods is not discussed in this review. With the development of nanotechniques, a series of new methods have been developed to fabricate nanomaterials with a variety of microstructures. Currently, it is possible to synthesize 2D compounds in large amounts to fabricate bulk thermoelectric materials. Since the grain boundary can scatter the phonons and can lead to low a thermal conductivity, it may be useful to fabricate high-performance thermoelectric materials by decreasing the particle size of the nanostructure thermoelectric material. Recently, 2D metal chalcogenides (including the group IV-VI compounds and the TMDCs) have triggered a considerable attention in the field of thermoelectrics as they exhibit a high ZT value. Due to their anisotropic crystal and electronic structures, these materials exhibit an intrinsically low thermal conductivity, which makes them promising thermoelectric materials $[17,150]$. 
Among the 2D metal chalcogenides thermoelectric materials, $\mathrm{SnSe}$ seems to be promising commercially available thermoelectric material. Until now, the highest $\mathrm{ZT}$ value for both the $p$-type ( 2.6 at $923 \mathrm{~K}$ ) and $n$-type (2.8 at $923 \mathrm{~K}$ ) compounds was obtained from SnSe single crystals. This may be due to their layered structure, soft chemical bonding, and lattice anharmonicity. However, the ZT value of polycrystalline SnSe bulk materials is lower than those of single-crystal ones. During the last several years, numerous efforts have been done to fabricate high-performance SnSe polycrystalline bulk thermoelectric materials. 2D SnSe samples with different morphologies can be synthesized by using different methods. Figure 4a-c shows typical 2D SnSe nanosheet and nanoplate morphologies [150-152]. For instance, Han et al. developed a surfactant-free solution-based method for the
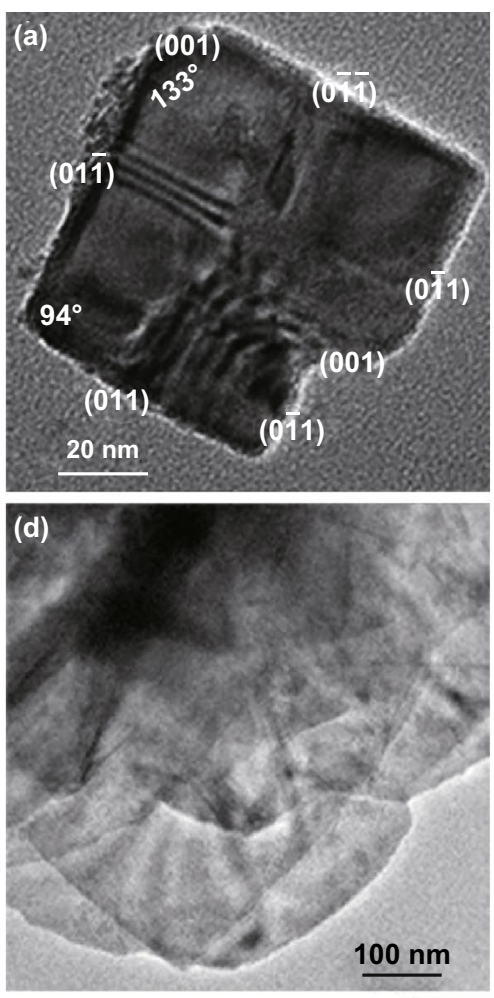

(g)

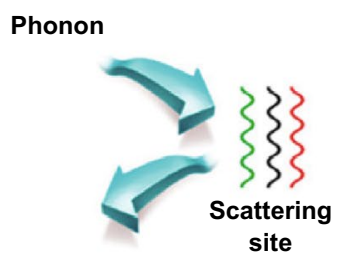

Phonon scattering
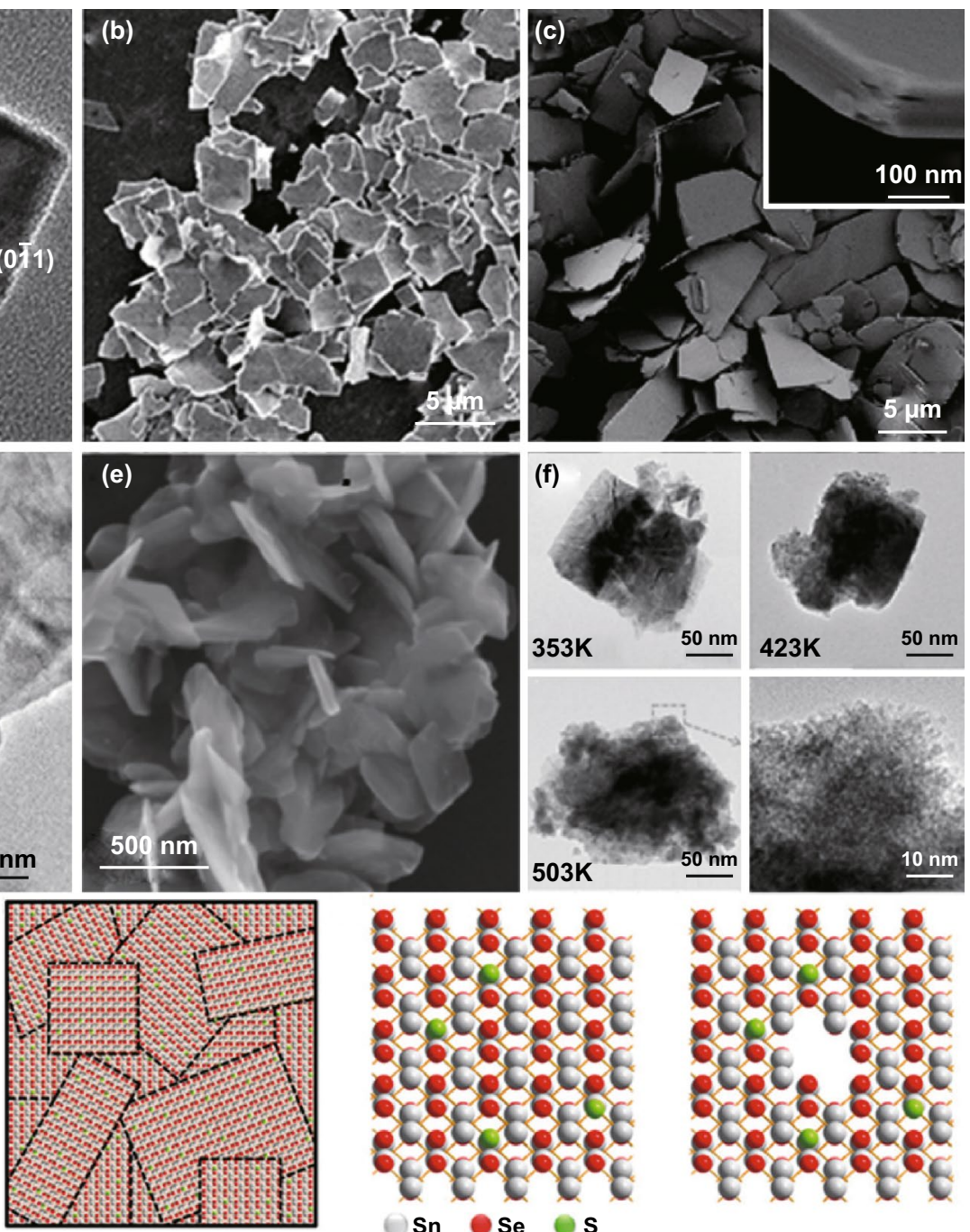

Nanoscale boundaries

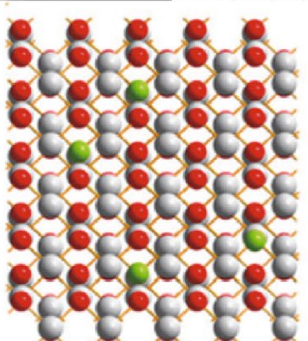

Sn Se os

Isoelectric atoms as dopants

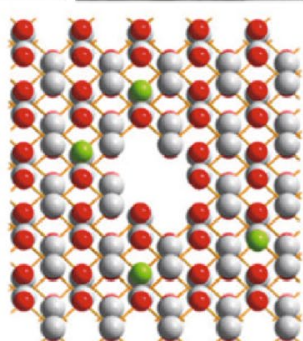

Introduction of pores

Fig. 4 a TEM image of a SnSe nanoplate. Reproduced with permission from Ref. [151]. Copyright 2016, Wiley-VCH. b SEM image of the

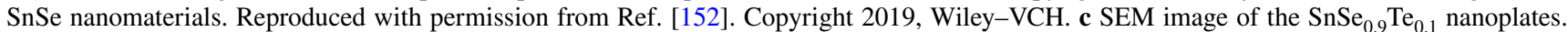
The inset shows the layer thickness. Reproduced with permission from Ref. [154]. Copyright 2017, Royal Society of Chemistry. d TEM image of the SnSe nanosheets. e SEM image of the $\mathrm{Sn}_{0.94} \mathrm{Bi}_{0.06} \mathrm{Se}$ nanosheets. Reproduced with permission from Ref. [150]. Copyright 2018, American Chemical Society. f Low-magnification FE-TEM images of the porous $\mathrm{SnSe}_{0.8} \mathrm{~S}_{0.2}$ NSs treated at the reaction temperatures of 353, 423, and $503 \mathrm{~K}$ and high-magnification FE-TEM image of the sample in the panel. $\mathrm{g}$ Schematic diagram of the phonon scattering mechanism of the $\mathrm{SnSe}_{0.8} \mathrm{~S}_{0.2}$ NSs. Reproduced with permission from Ref. [153]. Copyright 2017, American Chemical Society 
synthesis of single-phase SnSe nanoplates. Their power factor measured perpendicularly to the hot-pressing direction reaches $0.4 \mathrm{~mW} \mathrm{mK}^{-2}$ at $550 \mathrm{~K}$ [151]. Rongione et al. [152] fabricated a series of SnSe nanosheets and obtained an ultralow thermal conductivity of $0.09 \mathrm{~W} \mathrm{~m}^{-1} \mathrm{~K}^{-1}$. Such value mainly originates from the large thermal boundary resistance of the materials due to the strong phonon scattering near the interfaces between two SnSe nanosheets. In order to further improve the ZT value of SnSe bulk, $\mathrm{Bi}-$, Te-, and S- doped SnSe nanosheets were synthesized by using a large variety of methods [150, 153-155]. The SEM morphology of the doped SnSe samples is shown in Fig. 4d-f. Chandra et al. synthesized a 2D ultrathin $n$-type $\mathrm{Bi}$-doped $\mathrm{SnSe}$ nanosheet via a simple low-temperature solvothermal method [150]. The thickness of the SnSe nanosheet measures about 1-3 nm. Due to the presence of the nanoscale grain boundaries and the layered anisotropic structure, the heat-carrying phonons strongly scatter. This leads to an ultralow lattice thermal conductivity of $0.3 \mathrm{~W} \mathrm{mK}^{-1}$ in the range of $300-720 \mathrm{~K}$. Ju et al. fabricated a porous $\mathrm{SnSe}_{1-x} \mathrm{~S}_{x}$ nanosheet, and the details of this structure are shown in Fig. 4g [153]. A reduction in the thermal conductivity and an improved $\mathrm{ZT}$ value were obtained. Such enhanced ZT value is mostly due to the substitution of the $\mathrm{S}$ atoms into $\mathrm{SnSe}$, which induces the scattering of the phonons and several atomic disorders and nanosized boundaries. The mechanism of phonon scattering in porous $\mathrm{SnSe}_{0.8} \mathrm{~S}_{0.2}$-based materials is illustrated by the simple schematic diagram in Fig. $4 \mathrm{~g}$. As a result, the high ZT value of the bulk based on porous $\mathrm{SnSe}_{0.8} \mathrm{~S}_{0.2}$ nanosheets reaches 0.12 at $310 \mathrm{~K}$. The substitution of the $\mathrm{S}$ atoms into $\mathrm{SnSe}$ and the fabrication of the $\mathrm{SnSe}_{1-x} \mathrm{~S}_{x} \mathrm{NSs}$ reduce the thermal conductivity by introducing phonon scattering in the atomic disorders and nanoscale boundaries, leading to a higher $\mathrm{ZT}$.

Similarly to $\mathrm{SnSe}, \mathrm{SnSe}_{2}$ is also a layered structure thermoelectric material and exhibits promising thermoelectric performance [156-159]. Saha et al. fabricated ultrathin Cl-doped $\mathrm{SnSe}_{2}$ nanosheets with the thickness of 3-5 nm via a simple low-temperature solvothermal process [159]. Similarly to $\mathrm{SnSe}$, the hot-pressed $\mathrm{SnSe}_{2}$ pellet achieves an ultralow thermal conductivity of $0.67 \mathrm{~W} / \mathrm{mK}$ due to its effective phonon scattering at the grain boundary, which is induced by its anisotropic layered structure.

During the last decades, the most efficient single-phase thermoelectric materials used in the near room temperature range are $\mathrm{Bi}_{2} \mathrm{Te}_{3}$-based alloys, which are also layered materials. Until now, the optimized ZT value of these compounds measure around 1.0. The highest $\mathrm{ZT}$ value for the p-type and n-type $\mathrm{Bi}_{2} \mathrm{Te}_{3}$ alloys is obtained in the case of $\mathrm{Bi}_{0.5} \mathrm{Te}_{1.5} \mathrm{Se}_{3}$ $\left(\mathrm{ZT}_{\max }=1.2\right)$ and $\mathrm{Bi}_{2} \mathrm{Te}_{2.7} \mathrm{Se}_{0.3}\left(\mathrm{ZT}_{\max }=0.9\right)$ [17, 160]. Both these samples are bulk materials. Recently, $\mathrm{Bi}_{2} \mathrm{Te}_{3}$-based nanosheets have been widely reported in the fabrication of bulk $\mathrm{Bi}_{2} \mathrm{Te}_{3}$-based thermoelectric materials. As shown in Fig. 5a, Son et al. synthesized an ultrathin $\mathrm{Bi}_{2} \mathrm{Te}_{3}$ nanoplate with a thickness of about $1 \mathrm{~nm}$ via a simple solution process [161]. The $\mathrm{Bi}_{2} \mathrm{Te}_{3}$ bulk was prepared by sintering $\mathrm{Bi}_{2} \mathrm{Te}_{3}$ nanoplates via spark plasma sintering, and the maximum ZT value obtained was 0.62 at $400 \mathrm{~K}$. Figure $5 \mathrm{~b}$, c shows the TEM images of the $\mathrm{Bi}_{2} \mathrm{Se}_{3} @ \mathrm{Bi}_{2} \mathrm{Te}_{3}$ heterostructure nanoplates and of the $\mathrm{Bi}_{2} \mathrm{Se}_{3} @ \mathrm{Bi}_{2} \mathrm{Te}_{3} @ \mathrm{Bi}_{2} \mathrm{Se}_{3} @ \mathrm{Bi}_{2} \mathrm{Te}_{3}$ multishell nanoplates, which were scalable synthesized via a solution epitaxial growth process [162]. The thickness of the nanoplate is in the $5-20 \mathrm{~nm}$ range. Via the simultaneous modulation of the electronic and thermal transport in the presence of highly dense grain and phase boundaries, the peak ZT value measures 0.71 and it was obtained at $450 \mathrm{~K}$ for via the bulk sintering of the $\mathrm{Bi}_{2} \mathrm{Se}_{3} @ \mathrm{Bi}_{2} \mathrm{Te}_{3}$ nanoplates. Hong et al. synthesized a $n$-type $\mathrm{Bi}_{2} \mathrm{Te}_{3-x} \mathrm{Se}_{x}$ nanoplate by using the microwave-assisted surfactant-free solvothermal method. The TEM images of the nanoplate are shown in Fig. 5d, e [163]. The schematic diagram of the phonon scattering is shown in Fig. 5f, g. The grain boundaries, the point defects, and the dislocations may lead to the reduction in the lattice thermal conductivity. The experimental results prove these predictions. In this case, a relatively high $\mathrm{ZT}$ value of 1.23 at $480 \mathrm{~K}$ for the n-type $\mathrm{Bi}_{2} \mathrm{Te}_{2.7} \mathrm{Se}_{0.3}$ nanostructures was obtained from the sintered pellets. Since the nanostructure was employed as a raw material to obtain the sinter pellets, a high amount of grain boundaries was maintained and substantially reduced the thermal conductivity of the sample [163].

In the case of the bulk layered thermoelectric materials, the layered structure induces strong anisotropic thermoelectric properties along three different directions. More importantly, the grain boundaries, which were introduced by the nanostructuring, can substantially reduce the thermal conductivity when compared to the pellet structure. These samples were, in fact, synthesized by using ball milling and other melting methods. The 2D nanostructure materials employed to fabricate bulk thermoelectric materials further enhance their ZT value. 

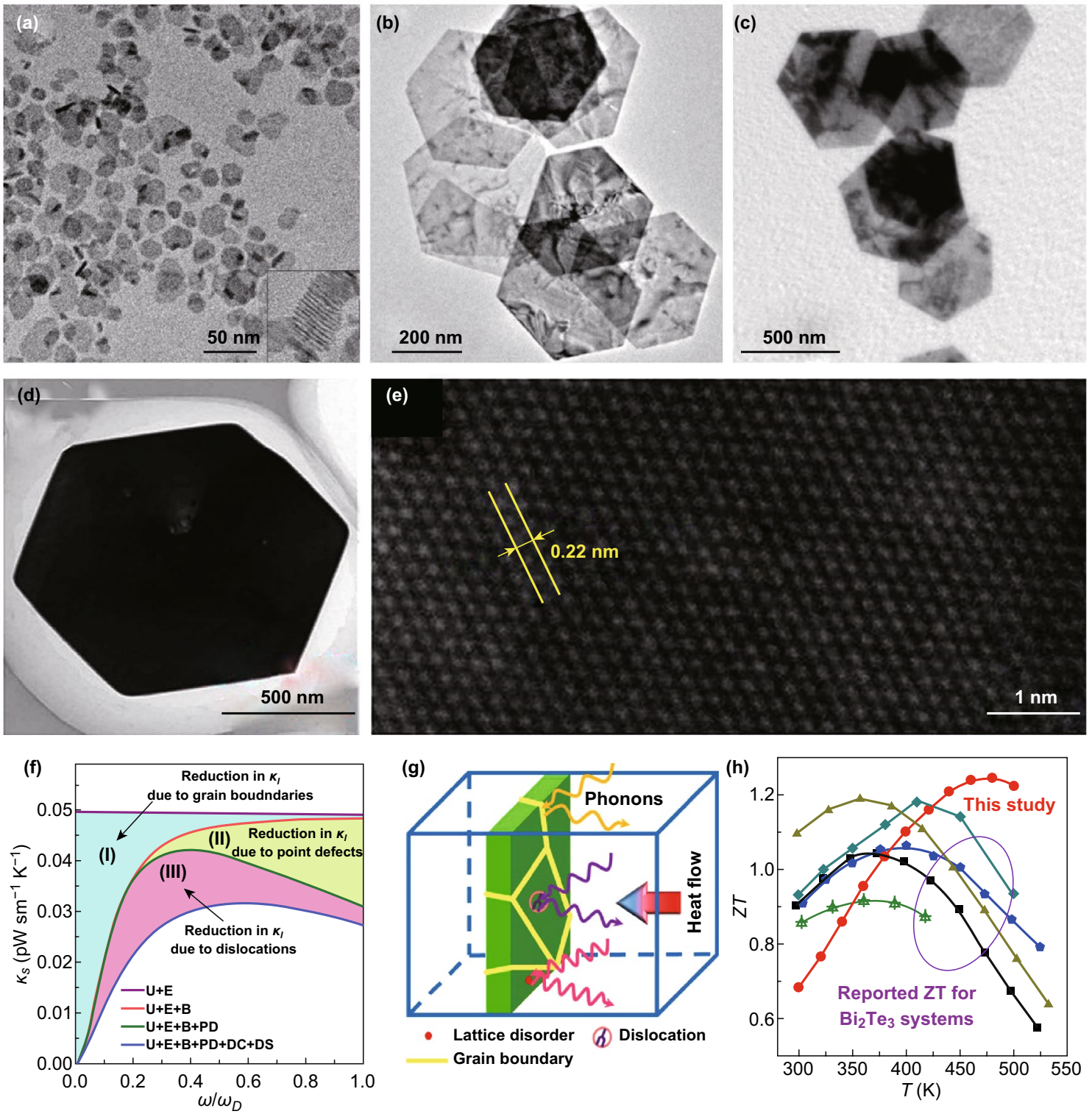

Fig. 5 a TEM image of the $\mathrm{Bi}_{2} \mathrm{Te}_{3}$ nanoplate, the inset shows the lateral view of the stacked plates). Reproduced with permission from Ref. [161]. Copyright 2012, American Chemical Society. b Bright-field TEM image of the $\mathrm{Bi}_{2} \mathrm{Se}_{3} @ \mathrm{Bi}_{2} \mathrm{Te}_{3} @ \mathrm{Bi}_{2} \mathrm{Se}_{3}$ double shell nanoplates. c TEM image of the $\mathrm{Bi}_{2} \mathrm{Se}_{3} @ \mathrm{Bi}_{2} \mathrm{Te}_{3} @ \mathrm{Bi}_{2} \mathrm{Se}_{3} @ \mathrm{Bi}_{2} \mathrm{Te}_{3}$ multishell nanoplates. Reproduced with permission from Ref. [162]. Copyright 2015, American Chemical Society. d TEM image, and e HRTEM image of the $\mathrm{Bi}_{2} \mathrm{Te}_{2.7} \mathrm{Se}_{0.3}$ nanoplate. $\mathbf{f}$ Calculated room-temperature $\kappa_{\mathrm{s}}$ value for the phonons scattering of the $\mathrm{Bi}_{2} \mathrm{Te}_{27} \mathrm{Se}_{0.3}$ pellet. $\mathbf{g}$ Schematic diagram illustrating the scattering of wide-frequency phonons by various sources. $\mathbf{h}$ Carriers mobility and ZT value of the $\mathrm{Bi}_{2} \mathrm{Te}_{2.7} \mathrm{Se}_{0.3}$ pellet. Reproduced with permission from Ref. [163]. Copyright 2016, American Chemical Society

\subsection{Thin-Film Thermoelectric Materials}

Thin-film thermoelectric materials have attracted a large attention due to their potential application as flexible and wearable devices. Besides the investigations on polymer-based thin-film thermoelectric materials, the more recent research efforts have been focused on thin-film thermoelectric materials fabricated by using nanostructures. Such newly developed 2D materials, such as graphene, BP, TMDCs, and group IV-VI compounds, can be easily 
synthesized and manufactured into thin films via various methods. As previously mentioned, the quantum size effect induced in the 2D nanostructure materials can increase the Seebeck coefficient. Moreover, the phonon scattering at the interfaces may lead to the a decrease in the thermal conductivity. The methods used to fabricate thin-film thermoelectric materials include vacuum evaporation, pulsed laser deposition, molecular beam epitaxy, magnetron sputtering, drop casting, spin coating, and inkjet printing [164-166].

Due to its unique electronic and optical properties, 2D BP has been widely studied in many research fields [114, 120, 122-124, 167-174]. Furthermore, it has been reported that it exhibits an ultrahigh Seebeck coefficient in the order of $10 \mathrm{mV} \mathrm{K}^{-1}$, which makes it a promising thermoelectric materials. Flores et al. studied the thermoelectric properties of bulk BP and found that its Seebeck coefficient measures about $335 \mu \mathrm{V} \mathrm{K}^{-1}$ at room temperature [130]. These results are lower than the theoretical predict data. Due to the poor stability of 2D BP, it is difficult to fabricate thermoelectric devices based on pristine BP. An et al. [129] decorated the BP surface with Au nanoparticles to enhance its thermoelectric properties and stability. The characterization of such structure is shown in Fig. 6a-c and its thermoelectric properties in Fig. 6d, e. The highest value of the Seebeck coefficient of this sample reaches $498 \mu \mathrm{V} \mathrm{K}^{-1}$ and the highest power factor is $68.5 \mu \mathrm{W} \mathrm{mK}{ }^{-2}$, which is about 2740 times higher than that of pristine BP.

Recently, 2D TMDCs have collected a wide attention due to their special properties and are considered to be the next generation of high-performance thermoelectric materials [70, 74, 90, 175-180]. The measured Seebeck coefficient of single-layer $\mathrm{MoS}_{2}$ can measure over $30 \mathrm{mV} \mathrm{K}^{-1}$, providing an ideal candidate material for high-performance thermoelectric devices. Huang et al. studied the thermoelectric properties of metallic 1T-phase $\mathrm{MoS}_{2}$ nanosheets. These $\mathrm{MoS}_{2}$ nanosheets were synthesized via a chemically exfoliated process, and the thin films were fabricated via a simple vacuum-assisted filtration process, as shown in Fig. 7a-d [181]. The schematic diagram of the crystal structure and the band structure of the $\mathrm{MoS}_{2}$ monolayer were calculated. By studying the band structure of the $2 \mathrm{H}$ and of the $1 \mathrm{~T} \mathrm{MoS}{ }_{2}$ monolayers, the high thermoelectric performance of the $\mathrm{MoS}_{2}$ film can be attributed to the metallic characteristic and to the conductivity nature of the $1 \mathrm{~T}$ phase $\mathrm{MoS}_{2}$. As shown in Fig. 7e, g, the power factor of the $\mathrm{MoS}_{2}$ film reaches $73.1 \mu \mathrm{W} \mathrm{mK}{ }^{-2}$ and exhibits an outstanding stability. However, the thermoelectric performance of $\mathrm{MoS}_{2}$ can be further enhanced by introducing several modifications. For instance, when the material is decorated with Au nanoparticles, the power factor of $\mathrm{MoS}_{2}$ increases to about $166.3 \mu \mathrm{W} \mathrm{mK}{ }^{-2}$ [182].

$\mathrm{TiS}_{2}$ is another widely studied $2 \mathrm{D}$ thermoelectric materials [13, 183-186]. As shown in Fig. 8a, Wan et al. [186] introduced a novel electrochemical intercalation and solvent exchange process to synthesize a $n$-type $\mathrm{TiS}_{2}\left[(\text { hexylammonium })_{x}\left(\mathrm{H}_{2} \mathrm{O}\right)_{y}(\mathrm{DMSO})_{z}\right]$ hybrid superlattice. The organic cations were distributed onto the two sides of each $\mathrm{TiS}_{2}$ layer. As shown in Fig. 8c-f, an electrical conductivity of $790 \mathrm{~S} \mathrm{~cm}^{-1}$ and a very high power factor of $0.45 \mathrm{~mW} \mathrm{mK}^{-1}$ were obtained in the case of the hybrid superlattice of $\mathrm{TiS}_{2} /\left[(\text { hexylammonium })_{x}\left(\mathrm{H}_{2} \mathrm{O}\right)_{y}(\mathrm{DMSO})_{z}\right]$. Moreover, its in-plane lattice thermal conductivity measures about $0.12 \mathrm{~W} \mathrm{mK}^{-1}$. This value is two orders of magnitude lower than that of single-layer and bulk $\mathrm{TiS}_{2}$. A high ZT value of 0.28 at $373 \mathrm{~K}$ was reported due to the high power factor and low thermal conductivity of the superlattice. This material exhibits an enormous potential for its application in wearable electronics devices. Tian et al. [184] fabricated a $\mathrm{TiS}_{2}$ /organic superlattice and tried to manufacture a $\mathrm{P}-\mathrm{N}$ prototype device. The devices with $5 \mathrm{P}-\mathrm{N}$ legs can generate an output voltage of $33 \mathrm{mV}$ with a maximum power density of $2.5 \mathrm{~W} \mathrm{~m}^{-2}$. The thermoelectric performance of $\mathrm{TiS}_{2}$ can also be enhanced via a chemical welding process. As shown in Fig. 8g, i, when the $\mathrm{TiS}_{2}$ nanosheets bridge with the multivalent cationic metal, $\mathrm{Al}^{3+}$, during the film deposition process, the Seebeck coefficient and the electrical conductivity can be improved simultaneously, thus leading to an enhanced power factor [183].

\section{2D Materials as Composite Filler}

Composite engineering has been considered the simplest and most efficient way to enhance the performance of thermoelectric materials since the formative crystallite boundaries scatter phonons effectively and decrease the thermal conductivity of the material. Moreover, the transport properties can be optimized if the filler is distributed in the matrix with a proper amount. In addition, it is possible to decouple the three parameters and to enhance the thermoelectric performance of the materials. 

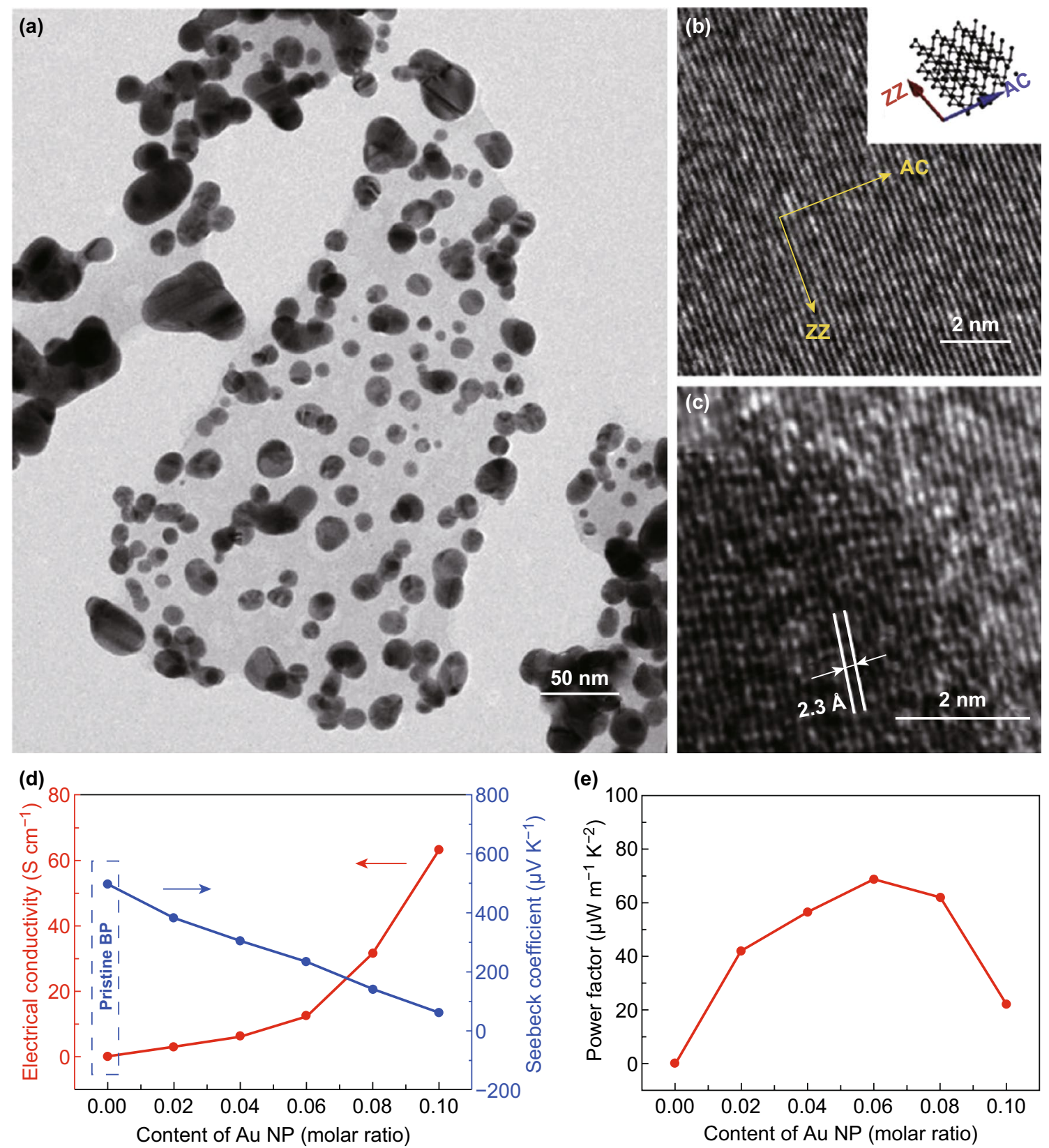

Fig. 6 a Low-magnitude TEM image of the black phosphorus nanosheet decorated with Au nanoparticles. b Lattice image of the exfoliated black phosphorus nanosheet (inset illustration showing the corresponding crystal structure). c High magnitude HRTEM image of the black phosphorus nanosheet decorated with Au nanoparticles. d In-plane Seebeck coefficient, electrical conductivity, and e their corresponding in-plane power factor of the black phosphorus decorated with Au nanoparticles. Reproduced with permission from Ref. [129]. Copyright 2018, Wiley$\mathrm{VCH}$ 


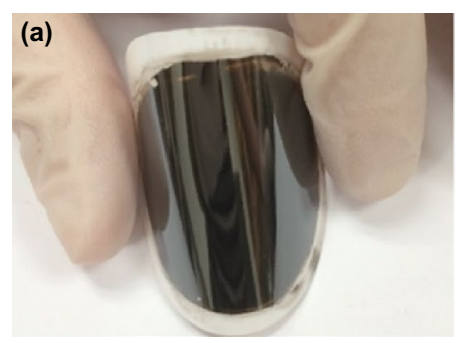

(e)

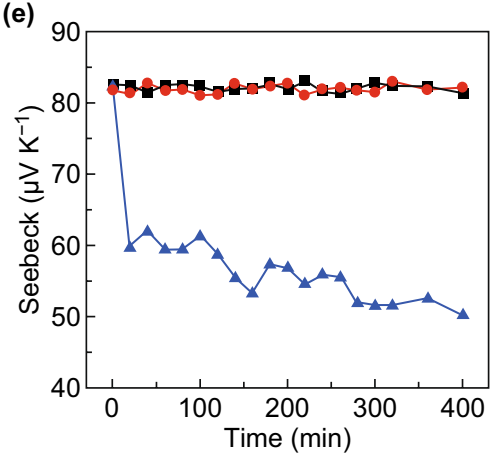

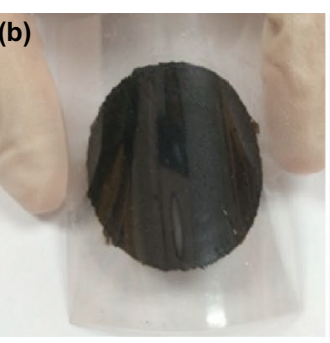

(f)

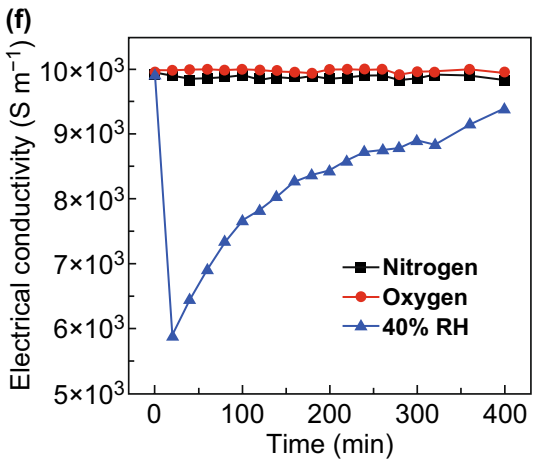

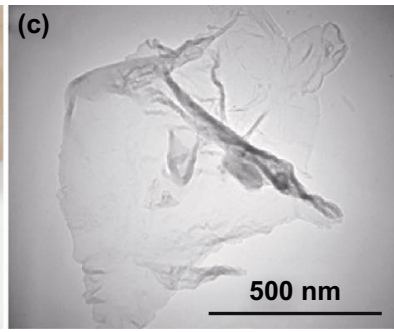

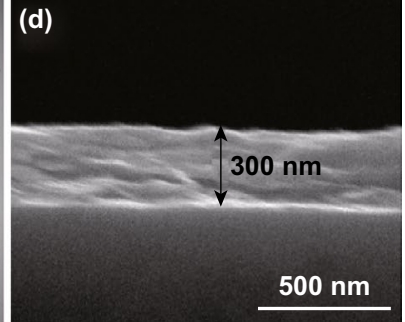

(g)

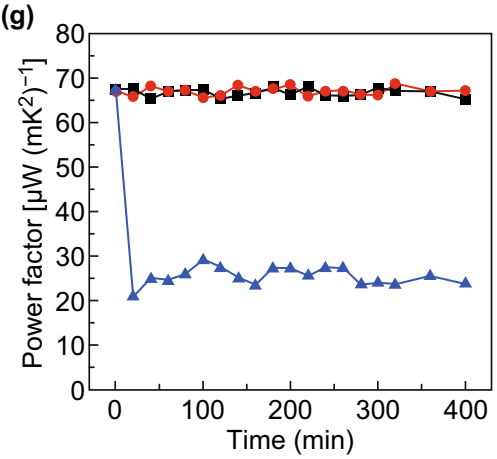

Fig. $7 \mathrm{MoS}_{2}$ nanosheets restacked film a on a cellulose ester membrane and $\mathbf{b}$ on a PET substrate. c TEM image of a single exfoliated MoS 2 nanosheet and $\mathbf{d}$ cross-sectional SEM image of the $\mathrm{MoS}_{2}$ nanosheets restacked film. e Dynamic Seebeck coefficient, $\mathbf{f}$ electrical conductivity, and g power factor of the $\mathrm{MoS}_{2}$ nanosheets restacked film in a nitrogen, oxygen and $40 \%$ relative humidity atmosphere. Reproduced with permission from Ref. [181]. Copyright 2016, Elsevier

\subsection{D Materials in Inorganic Bulk Thermoelectric Materials}

The fabrication of hybrid composites is one of the most important methods to improve the thermoelectric performance of bulk thermoelectric materials, in which the nanomaterials are either located at the grain boundary or homogeneously dispersed in the matrix. The matrix materials form a continuous conductive network to maintain the same electrical conductivity within the matrix. Moreover, the dispersed second phase present in the matrix can scatter the phonons. The addition of a 2D material introduces a heterojunction interface, which can scatter the carrier and decrease its mobility, thus leading to a further enhancement of the thermoelectric performance. By adding a nanofiller added into the matrix, the relation among the electrical conductivity, the Seebeck coefficient, and the thermal conductivity can be decoupled by modulating the electron and phonon-transport characteristics.

In order to further enhance the thermoelectric properties of conventional thermoelectric materials, several kinds of 2D materials have been added into the bulk to fabricate different composites. Upon the addition of 2D materials filler, the carrier and the phonon-transport characteristics can be modulated. Besides, the addition of a 2D material introduces a heterojunction interface, which can scatter the carrier and decrease its carrier mobility, thus leading to an enhancement in the thermoelectric performance of the compound.

Graphene has been widely investigated in many fields due to its excellent properties. Its high Seebeck coefficient (about $30 \mathrm{mV} \mathrm{K}^{-1}$ ), ultrahigh electrical conductivity $\left(>14 \mathrm{~S} \mathrm{~cm}^{-1}\right)$, large charge-carrier mobility $\left(>2 \times 10^{5} \mathrm{~cm}^{2}(\mathrm{Vs})^{-1}\right)$, and extraordinary electronic transport properties make it an ideal candidate for the fabrication of high-performance thermoelectric materials. In the past years, graphene was chosen as a composite filler to fabricate high-performance bulk thermoelectric composites. In 2015, Lin et al. fabricated a graphene/lanthanum strontium titanium oxide (LSTO) composite [187]. Upon the addition of a small amount of graphene, the thermal operation window of LSTO decreases to room temperature. As shown in Fig. 9, the highest $\mathrm{ZT}$ value is about 0.42 at room temperature and 0.36 at $750{ }^{\circ} \mathrm{C}$ was obtained when a quantity of $0.6 \%$ of graphene was added into the samples. Chen et al. successfully modulated the carrier concentrations, the electrical conductivity, and the thermal conductivity by adding reduced graphene oxide 

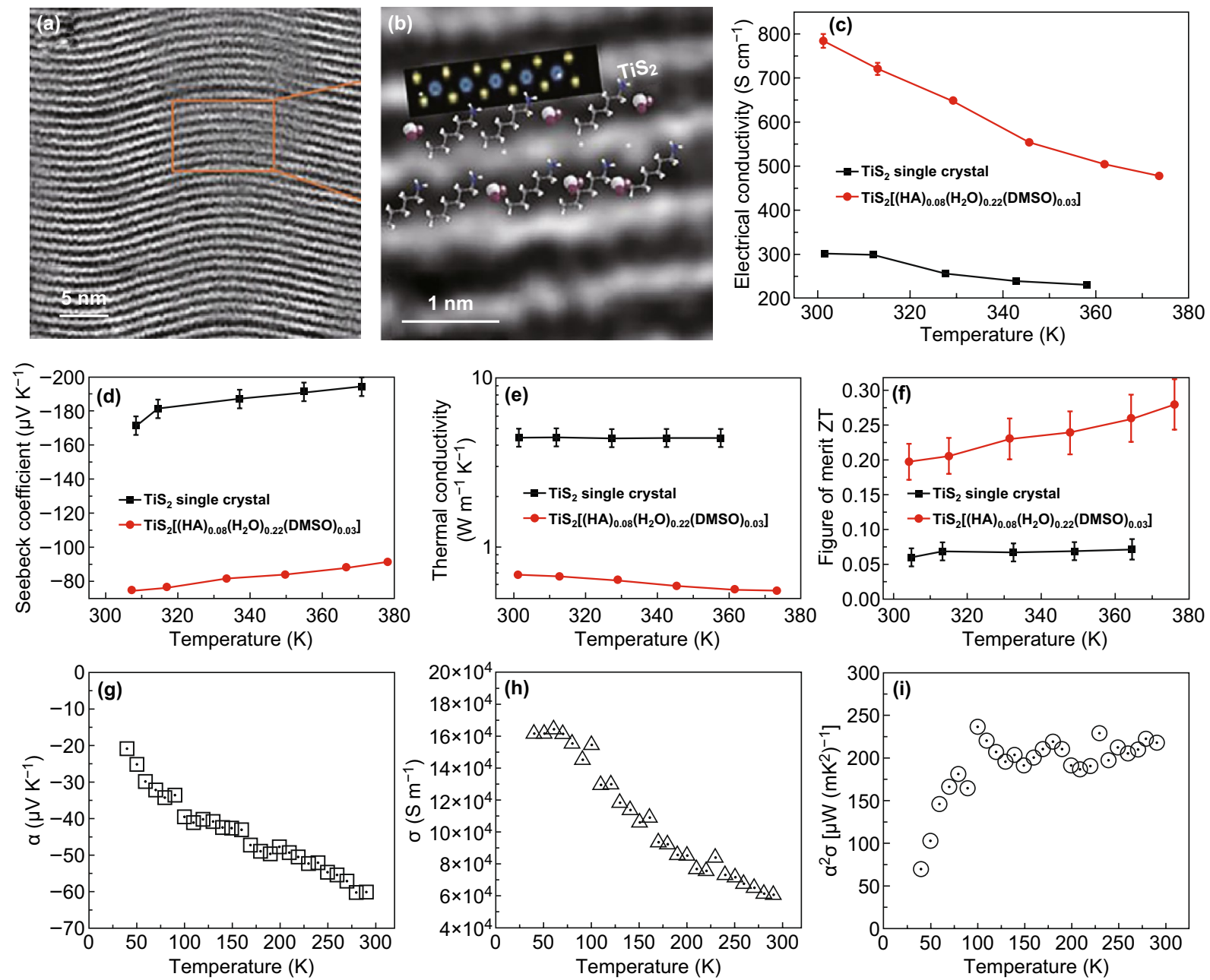

Fig. 8 a, b HAADF-STEM image of the $\operatorname{TiS}_{2}\left[(\mathrm{HA})_{x}\left(\mathrm{H}_{2} \mathrm{O}\right)_{y}(\mathrm{DMSO})_{z}\right]$ hybrid superlattice, c electrical conductivity, d Seebeck coefficient, e thermal conductivity, and $\mathbf{f} \mathrm{ZT}$ of the $\mathrm{TiS}_{2}\left[(\mathrm{HA})_{x}\left(\mathrm{H}_{2} \mathrm{O}\right)_{y}(\mathrm{DMSO})_{z}\right]$ hybrid superlattice. Reproduced with permission from Ref. [186]. Copyright 2015, Springer Nature. $\mathbf{g}$ Seebeck coefficient, h electrical conductivity, and i power factor of the $\mathrm{TiS}_{2}$ nanosheet assembled thin film. Reproduced with permission from Ref. [183]. Copyright 2017, American Chemical Society

into the $\mathrm{Al}$-doped $\mathrm{ZnO}$ [188]. Since the carrier concentration and the electrical conductivity of graphene oxide are much larger than those of $\mathrm{ZnO}$, the nanocomposite exhibits an enhanced electrical conductivity when compared to pristine $\mathrm{Al}$-doped $\mathrm{ZnO}$. More importantly, although the thermal conductivity of graphene oxide is much higher than that of $\mathrm{ZnO}$ and $\mathrm{Al}$-doped $\mathrm{ZnO}$, the total thermal conductivity and the lattice thermal conductivity show an obvious decrease, upon the addition of graphene. These results are shown in Fig. 9d. Li et al. [189] found that by adjusting the density and the dispersion manner of graphene in the bulk matrix, the thermoelectric performance of the composite could be further enhanced. Upon an increase in the graphene amount, the $\mathrm{Bi}_{2} \mathrm{Te}_{3}$ density at the interface increases. Moreover, the high density of the heterojunction interface may enhance carrier scattering and decrease its mobility, thus leading to a decrease in the electrical conductivity and in the thermal conductivity of the compound. In addition, the size of the dopant shows a pronounced effect on the carrier and phonon-transport characteristics. For example, as reported in Li's paper, the $\mathrm{Bi}_{2} \mathrm{Te}_{3}$ /graphene composite exhibits a highest $\mathrm{ZT}$ value $(\sim 0.55)$ at $425 \mathrm{~K}$ as the size of the graphene reaches $20 \mathrm{~nm}$, as shown in Fig. 9e [189]. The same result was also reported for other bulk thermoelectric material systems, such as $\mathrm{PbTe} /$ graphene composite [190], $\mathrm{Cu}_{2} \mathrm{SnSe}_{3} /$ graphene [191], $\mathrm{CuInTe}_{2}$ /graphene [192], $\mathrm{CoSb}_{3}$ /graphene 

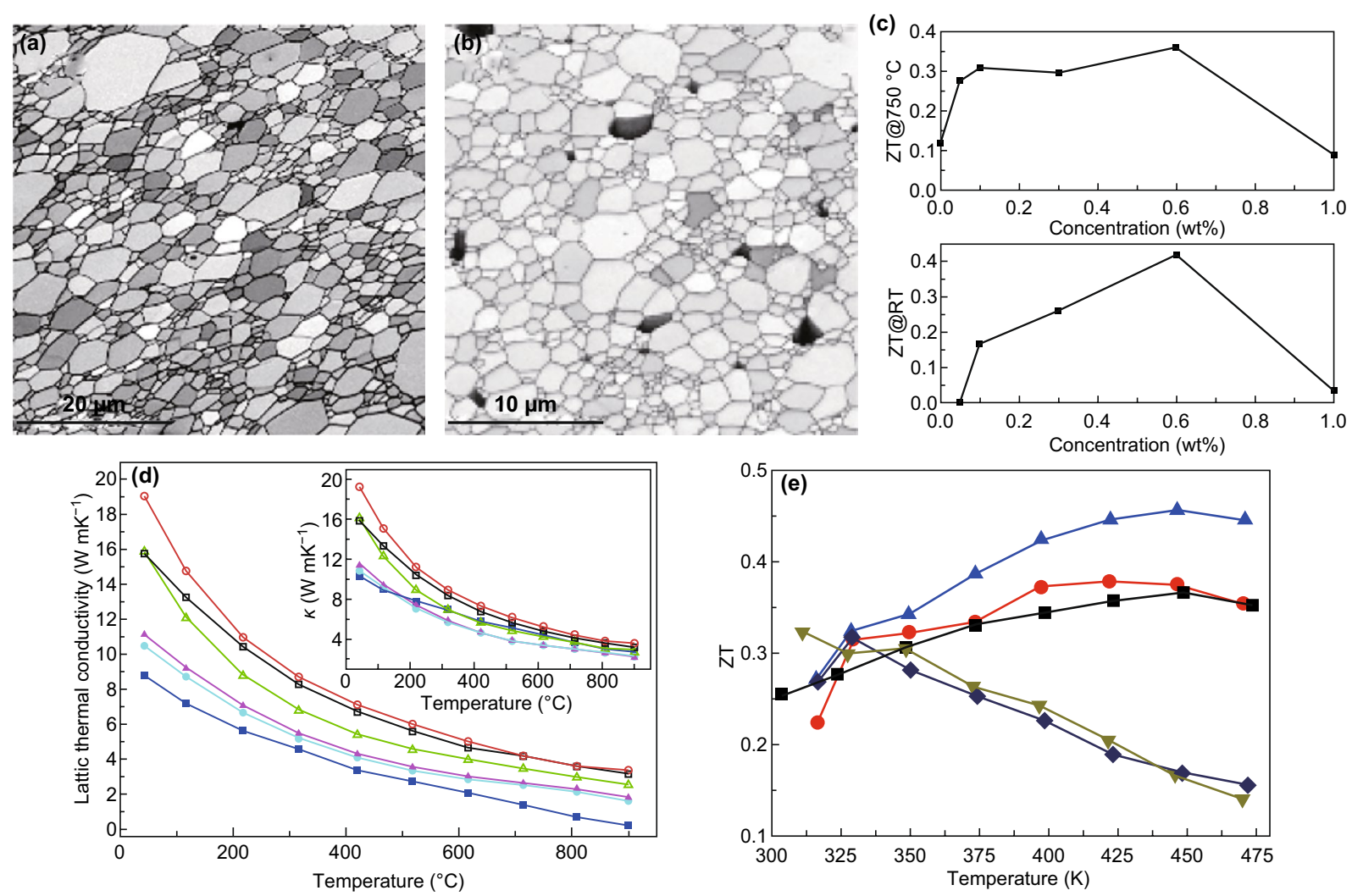

Fig. 9 EBSD analysis of lanthanum strontium titanium oxide sample, and graphene/lanthanum strontium titanium oxide. a Band contrast image of a pure lanthanum strontium titanium oxide sample; $\mathbf{b}$ band contrast image of $0.1 \mathrm{wt} \%$ graphene/lanthanum strontium titanium oxide. $\mathbf{c}$ ZT at room temperature and at $750{ }^{\circ} \mathrm{C}$ as a function of the graphene concentration. Reproduced with permission from Ref. [187]. Copyright 2015, American Chemical Society. d Lattice thermal conductivity and (inset) total thermal conductivity of the Al-doped $\mathrm{ZnO}$ and AZO/rGO samples. Reproduced with permission from Ref. [188]. Copyright 2015, American Chemical Society. e Thermoelectric properties of the $\mathrm{Bi}_{2} \mathrm{Te}_{3} / \mathrm{GQDS}$ samples with different GQD contents. Reproduced with permission from Ref. [189]. Copyright 2017, American Chemical Society

[193], $\mathrm{SnSe} / \mathrm{MoS}_{2} /$ graphene [194], and $\mathrm{Bi}_{0.5} \mathrm{Sb}_{1.5} \mathrm{Te}_{3}$ /graphene [195].

Besides graphene, TMDCs have also been widely used as efficient composite fillers. In 2017, Li et al. [196] partially decoupled the electrical conductivity, the Seebeck coefficient, and the thermal conductivity by adding $2 \mathrm{D} \mathrm{SnS}_{2}$ nanosheets into a $\mathrm{Bi}_{2} \mathrm{Te}_{2.7} \mathrm{Se}_{0.3}$ matrix. As shown in Fig. 10a, the $\mathrm{SnS}_{2}$ nanosheets homogenously assemble onto $\mathrm{Bi}_{2} \mathrm{Te}_{2.7} \mathrm{Se}_{0.3}$ grain boundaries and formed a nanoscale heterojunction interface. A ZT of 0.93 at $450 \mathrm{~K}$ was measured. Such high value was attributed to the optimized carrier and phonon-transport characteristics induced by the $\mathrm{SnS}_{2} / \mathrm{Bi}_{2} \mathrm{Te}_{2.7} \mathrm{Se}_{0.3}$ interface. Huang et al. [197] reported a high ZT value for a polycrystalline $\mathrm{SnSe}$ sample via the fabrication of a $\mathrm{MoSe}_{2} / \mathrm{SnSe}$ composite. Upon the addition of $2 \mathrm{D} \mathrm{MoSe}_{2}$ into the SnSe matrix, both the carrier concentration and the carrier mobility are significantly improved when compared to pure polycrystal SnSe, as shown in Fig. 10c, d. For the $\mathrm{MoSe}_{2} / \mathrm{SnSe}$ composite, the carrier concentration is about one order of magnitude higher than that of pure SnSe. Moreover, the carrier mobility of the composite shows an impressive enhancement. This phenomenon may be related to the carrier scattering, due to the energy barrier introduced at the heterojunction interface.

\subsection{D Materials in Polymer Thermoelectric Materials}

As a potential high-performance thermoelectric material, conductive polymers exhibit unique advantages such as a low thermal conductivity, flexibility, lightweight, and efficient solution processability [9, 198-202]. However, more work needs to be done to further improve their thermoelectric performance to use them in flexible and wearable thermoelectric devices. The power factor for typical 

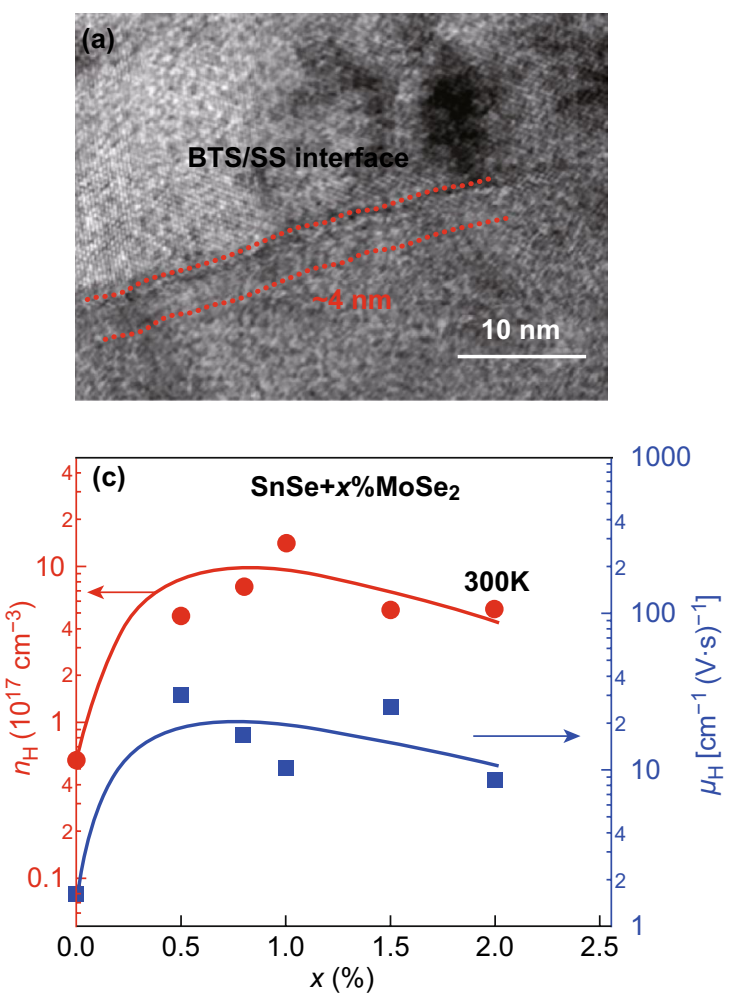
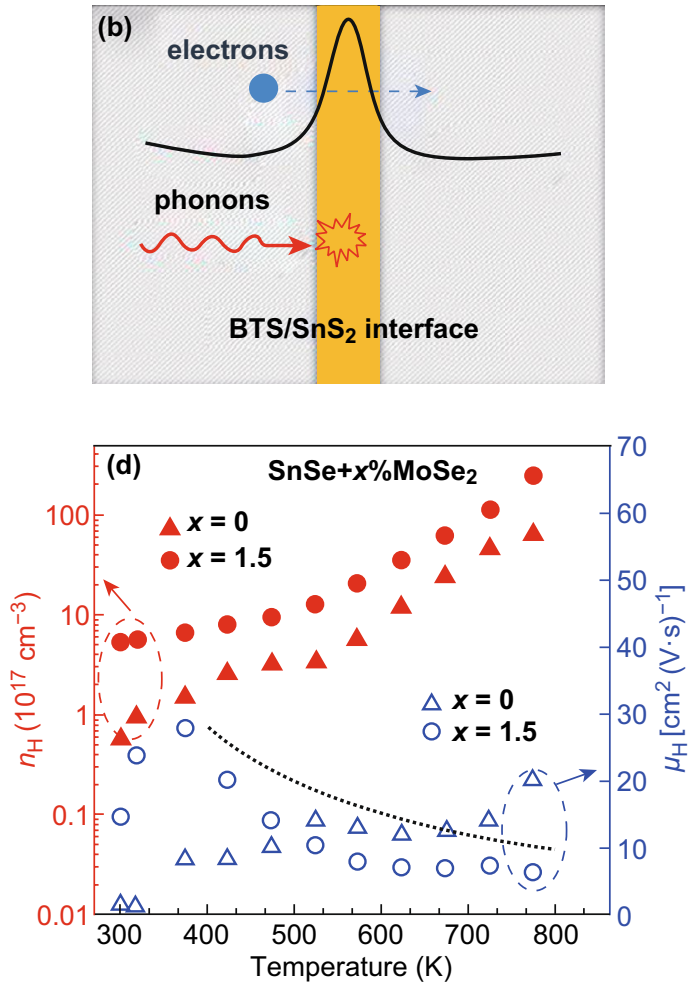

Fig. 10 a HRTEM image of the $\mathrm{Bi}_{2} \mathrm{Te}_{2.7} \mathrm{Se}_{0.3} / \mathrm{SnS}_{2}$ bulk; b mechanisms that contribute to the high $\mathrm{ZT}$ value of the $\mathrm{Bi}_{2} \mathrm{Te}_{2.7} \mathrm{Se}_{0.3} / \mathrm{SnS}_{2}$ nanocomposites. Reproduced with permission from Ref. [196]. Copyright 2017, Elsevier. c Carrier concentration and mobility of the SnSe/MoSe ${ }_{2}$ composites at $300 \mathrm{~K}$ as a function of the $\mathrm{MoSe}_{2}$ content. d Temperature dependence of carrier concentration and of the carrier mobility of SnSe/ $\mathrm{MoSe}_{2}$. Reproduced with permission from Ref. [197]. Copyright 2017, IOP Publishing

conductive polymer thermoelectric materials is in the $10^{-4}$ to $10^{2} \mu \mathrm{W} \mathrm{mK}^{-2}$ range at room temperature. This value is about 3 orders of magnitude lower than those of traditional inorganic thermoelectric materials [199, 203, 204]. According to the theoretical and experimental achievement, the fabrication of composite blends is an efficient way to improve the thermoelectric performance of conductive polymers.

In the last decade, a number of investigations have been reported to improve the thermoelectric performance of conductive polymers by adding a nanostructure filler. Polymer blends fabricated by employing highly electrical conductive constituents and high-Seebeck-coefficient constituents show an improvement in both their Seebeck coefficient and electrical conductivity. As previously discussed, 2D materials possess excellent electrical and mechanical properties, which are suitable to fabricate high-performance composite thermoelectric materials. In a simple 2D materials/polymer composite system, its thermoelectric performance of the composite can be evaluated based on either a serially or parallel connected model, if the interfaces are neglected [205,
206]. The simulation results indicate that the Seebeck coefficient and the electrical conductivity cannot exceed those of their individual components [207, 208]. However, recent advances in the theory have proven that the introduction of an energy filtering effect at the interface can overcome this limitation and can be experimentally proven [206, 207, 209].

2D materials with a high Seebeck coefficient have been widely employed as efficient fillers to enhance the thermoelectric performance of polymer blends. 2D materials were added into the polymer blends to increase their Seebeck coefficient. For instance, the group IV metal chalcogenides (e.g., SnSe, SnS) can be exfoliated into 2D materials. The crystal structures of SnSe along different directions at $300 \mathrm{~K}$ are shown in Fig. 11a. The strong Sn-Se bonds along the $b-c$ planes are connected with the weak $\mathrm{Sn}-\mathrm{Se}$ bonds along the $a$-axis of $\mathrm{SnSe}$, indicating that bulk SnSe can be easily exfoliated into 2D ultrathin materials along the a-direction. Ju et al. [205] prepared SnSe nanosheets via hydrothermal lithium-intercalation, which was then followed by an exfoliation process from 

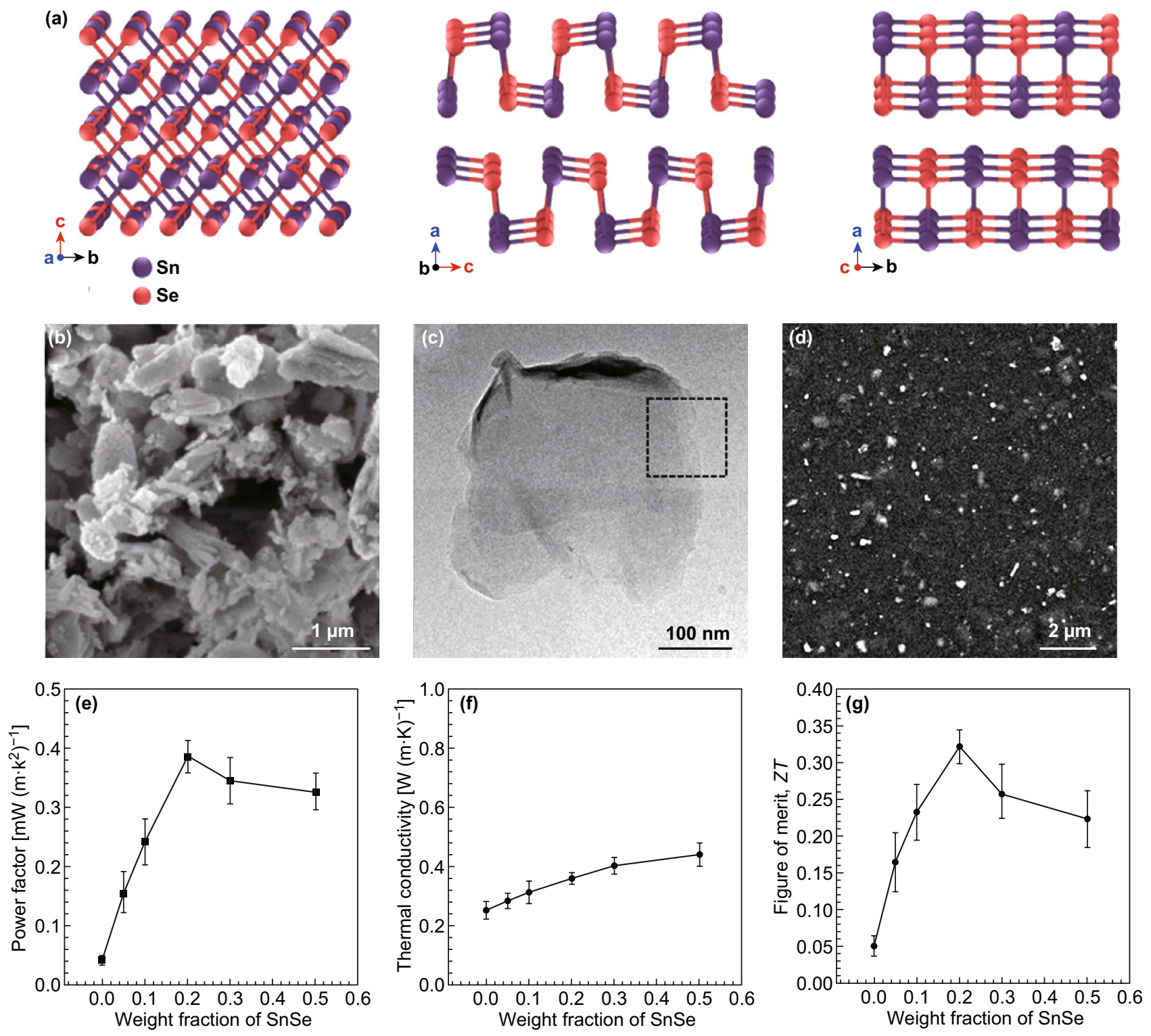

Fig. 11 a Crystal structures of SnSe along the $a$-, $b$-, and $c$ - axial directions. b SEM morphology and c TEM image of SnSe nanosheets. d SEM of the SnSe/PEDOT:PSS composites with SnSe a content of 50\%. e Power factor, $\mathbf{f}$ thermal conductivity, and $\mathbf{g}$ ZT value of the SnSe/ PEDOT:PSS composites. Reproduced with permission from Ref. [205]. Copyright 2016, American Chemical Society

the SnSe powders. During the intercalation process, ethylene glycol acts as both the solvent and the reducing agent. The morphology of the exfoliated SnSe nanosheets is shown in Fig. 11b-d. Their thickness is about $3.4 \mathrm{~nm}$. The Seebeck coefficient of pure $\mathrm{SnSe}$ at room temperature is higher than $520 \mu \mathrm{V} \mathrm{K} \mathrm{K}^{-1}$, whereas for PEDOT:PSS is about $30 \mu \mathrm{V} \mathrm{K} \mathrm{K}^{-1}$. As the SnSe nanosheets are dispersed into the PEDOT:PSS solution, the measured Seebeck coefficient increases with the increase in the weight fraction of
SnSe. The increase in the power factor to $386 \mu \mathrm{W} \mathrm{mK}^{-2}$ is induced by the substantial increases in the Seebeck coefficient when compared to the reduction in the electrical conductivity of the material. Other 2D group IV metal chalcogenides have also been fabricated and added as fillers to enhance the thermoelectric performance of the composites [210-213]. Ju et al. [212] coated the SnSeS nanosheet by polyaniline (PANI) and then used it as a filler to fabricate the composite. The PANI-coated SnSeS 


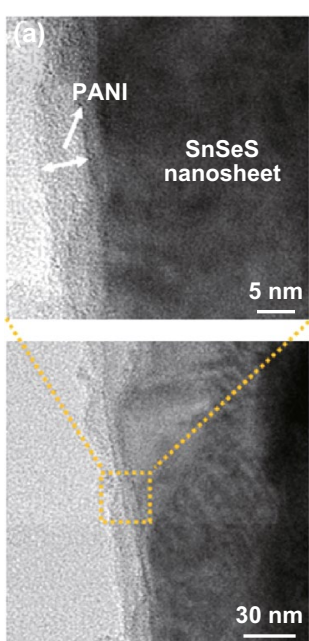

(b)

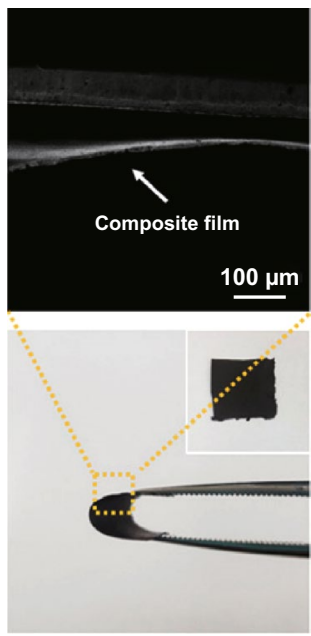

(d)
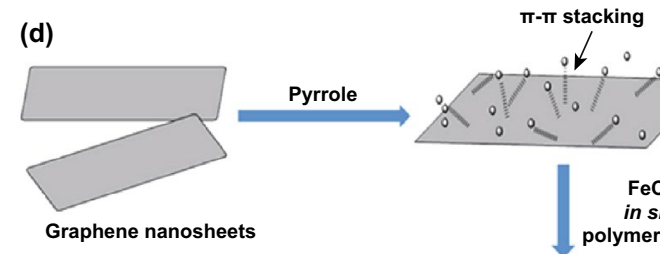

- is stacking

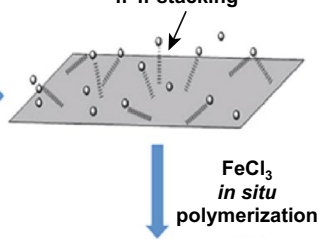

plymerization
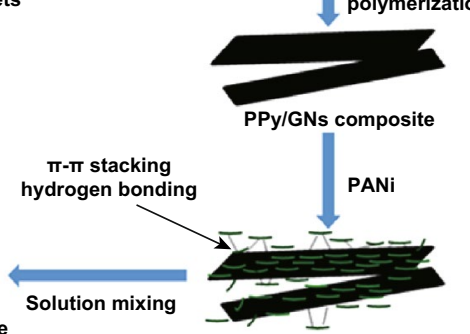

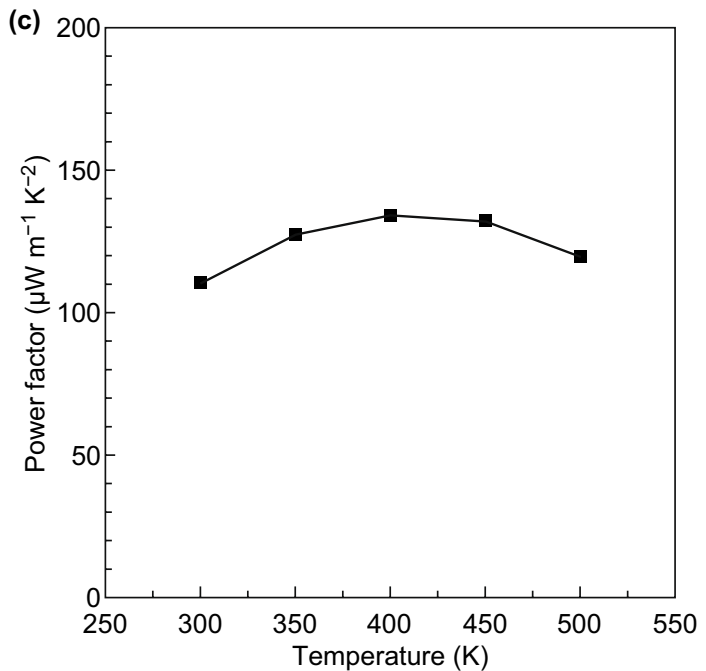

(e)

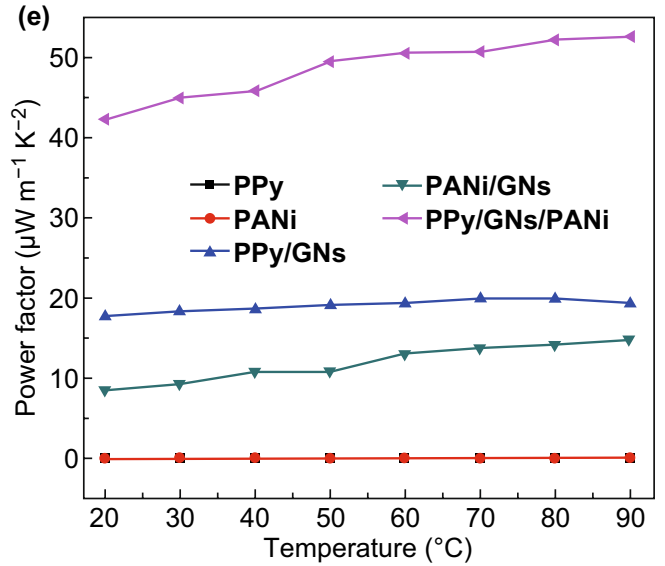

Fig. 12 a TEM image of the $\mathrm{SnSe}_{0.8} \mathrm{~S}_{0.2}$ nanosheets, $\mathbf{b} \mathrm{SEM}$ image of the PANI-coated $\mathrm{SnSe}_{0.8} \mathrm{~S}_{0.2}$ film and $\mathbf{c}$ power factor of the PANI-SnSeS nanosheet/PVDF composite film with a PANI-SnSeS nanosheet-to-PVDF ratio of 2:1 as a function of temperature. Reproduced with permission from Ref. [212]. Copyright 2018, American Chemical Society. d Schematic process of the polypyrrole/graphene/polyaniline ternary nanocomposite and e its power factor. Reproduced with permission from Ref. [220]. Copyright 2017, American Chemical Society

nanosheet added into polyvinylidene fluoride (PVDF) exhibits a maximum power factor of $134 \mu \mathrm{W} \mathrm{mK}^{-2}$ at $400 \mathrm{~K}$, as shown in Fig. 12a. SnS has also been chosen as a filler to improve the properties of polymer thermoelectric materials. Recently, Cheng et al. [214] have fabricated a SnS/PEDOT:PSS composite and showed that its Seebeck coefficient increases upon the increase in its SnS content. In 2019, Ju et al. fabricated PANI-coated porous SnS nanosheets and characterized its thermoelectric properties [214]. An outstanding ZT value of 0.078 at $450 \mathrm{~K}$ was obtained by adjusting the PANI coating layer.

Besides the group IV metal chalcogenides, TMDCs have also been widely studied as high-performance thermoelectric materials. TMDCs have gained considerable attention as a potential thermoelectric material in recent years due to their low thermal conductivity $\left(0.1-1 \mathrm{~W} \mathrm{mK}^{-1}\right)$ and their large in-plane mobility $\left(200-500 \mathrm{~cm}(\mathrm{Vs})^{-1}\right)$. Recently, several theoretical and experimental studies on the thermoelectric performance of TMDCs have been reported. Although the thermoelectric performance of the TMDCs-based bulk materials is limited due to their poor electrical conductivity, TMDCs with their large Seebeck coefficient are an ideal composite filler to fabricate high-performance polymer blends. In 2010, Zhang et al. [215] incorporated both a $n$-type and a $p$-type $\mathrm{Bi}_{2} \mathrm{Te}_{3}$ into a PEDOT:PSS solution and successfully fabricated both $p$-type and $n$-type polymer composite materials. However, by adjusting the morphology of the nanostructure, the thermoelectric performances 
of these composites were further enhanced. Du et al. [216] fabricated a $\mathrm{Bi}_{2} \mathrm{Te}_{3}$ nanosheet/PEDOT:PSS thin-film composite via a simple coating process. With the addition of $\mathrm{Bi}_{2} \mathrm{Te}_{3}$ nanosheets, the Seebeck coefficient and the electrical conductivity of the composite increase simultaneously. Generally, in this kind of composite materials, a 2D material filler is chosen to improve the Seebeck coefficient, thus leading to a high power factor. In 2016, Jiang et al. [217] fabricated a high-performance $\mathrm{MoS}_{2}$ /PEDOT:PSS thin film via a vacuum filtration process. By the addition of a small amount of liquid-phase exfoliated $\mathrm{MoS}_{2}$ nanosheets into a PEDOT:PSS solution, the thermoelectric properties of the PEDOT:PSS-based thin film were enhanced significantly. Several 2D nanostructures including nanosheet, nanoparticles, nanowire, and nanobarbell were reported to enhance

the thermoelectric performance of the films [205, 211, 212, 215-219].

Moreover, the addition of a high-Seebeck-coefficient component, high-electrical-conductivity component was also employed to enhance the electrical properties of the hybrid composite, leading to an enhanced power factor. However, 2D materials with a high electrical conductivity, such as graphene and reduced graphene oxide, have also been widely studied. Due to their relatively low electrical resistivity, this group of 2D materials is usually employed to enhance the electrical conductivity of the composite. As shown in Fig. 12c, Wang et al. [220] fabricated a polypyrrole/graphene/polyaniline ternary nanocomposite via a simple in situ polymerization process. Due to the ultrahigh electrical conductivity of graphene, this ternary composite

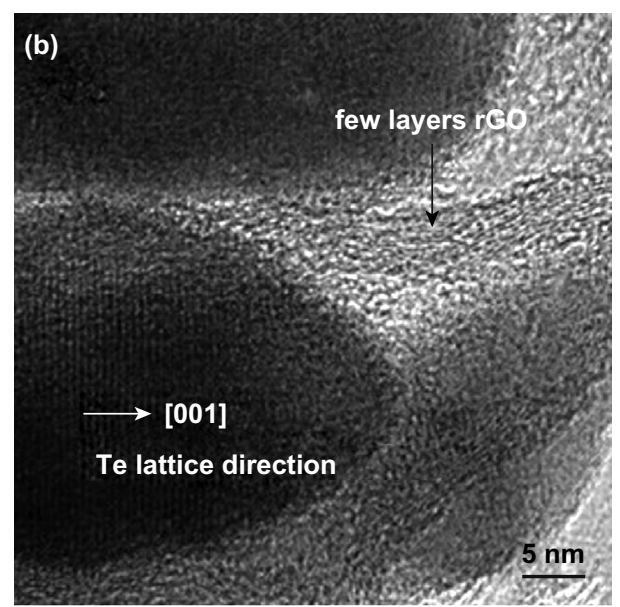

Ternary hybrid

(c)
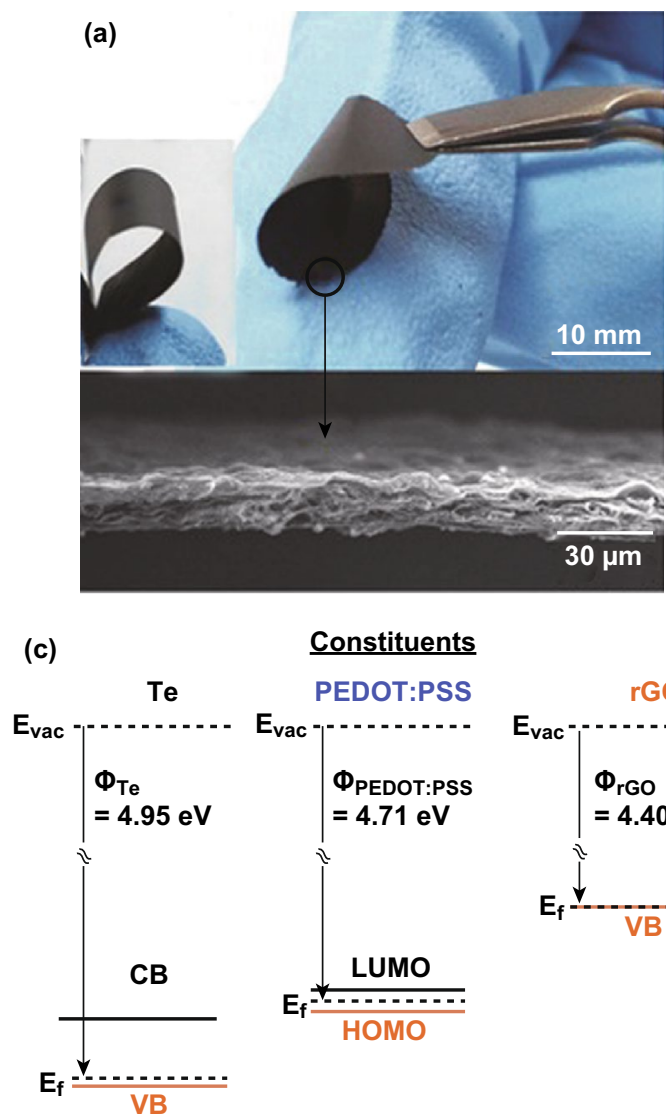

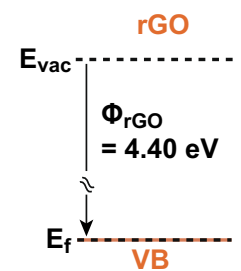

(d)

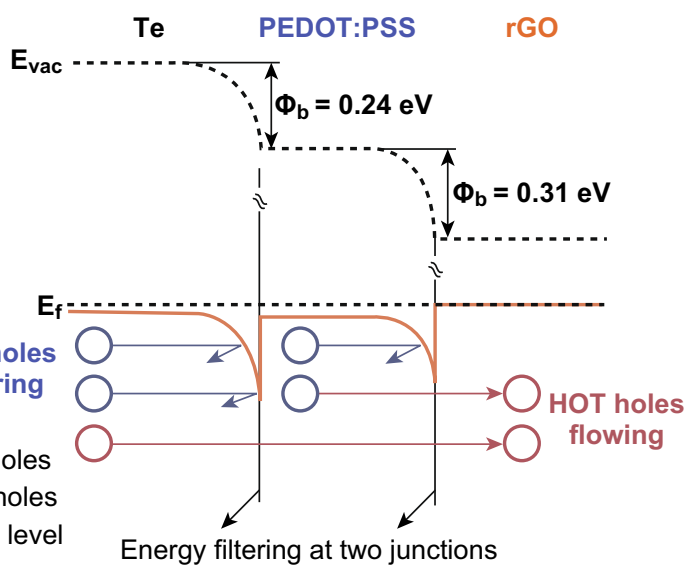

Fig. 13 a Photographs and cross-sectional SEM images of the flexible rGO/PEDOT:PSS/Te hybrid paper. b HRTEM images of the rGO/ PEDOT:PSS/Te hybrid composite. c Energy diagram of the rGO/PEDOT:PSS/Te heterojunctions. d Energy filtering effects at two junctions. Reproduced with permission from Ref. [208]. Copyright 2016, Wiley-VCH 
exhibits at least two magnitude higher Power factors than that of PANI and PPy. As shown in Fig. 13, Choi et al. [208] fabricated a novel Graphene/PEDOT:PSS/Te composite. The electrical conductivity of such composite is about 15 times higher than that of PEDOT:PSS/Te although the Seebeck coefficient is almost identical. The significant increase in the electrical conductivity of the ternary composite is ascribed to the carrier scattering at the double interface, as shown in Fig. 13c, d. This work shows that graphene may be an ideal composite filler to optimize the thermoelectric performance of polymers. Inspired by these pioneer works, several investigations, including graphene/ $\mathrm{P}_{3} \mathrm{HT}$, rGO/PEDOT:PSS, $\mathrm{PANI} /$ graphene and graphene/CNT, have reported the use of graphene or reduced graphene oxide into a conductive polymer [43, 59, 221-226].

Besides their use in the enhancement of either the Seebeck coefficient or the electrical conductivity, 2D materialbased composites can be employed as electrodes to fabricated high-performance flexible devices. Jiang et al. [227] prepared a graphene/polyethyleneglycol composite, which was used for heat collection and transport. The presence of highly conductive graphene in this composite introduces several conductive pathways for heat transfer and acts as a highly thermal conductive reservoir of phase-change materials for thermal energy collection, storage, and release. Moreover, this work opens a new door to fabricate novel thermoelectric materials and to realize high-performance flexible thermoelectric devices.

\section{Thermoelectric Properties of Single- or Few-layer 2D Materials}

In the past years, a significant effort has been made to improve the thermoelectric efficiency of the $2 \mathrm{D}$ materials. The most efficient ways to enhance their thermoelectric performance is developing novel materials and novel nanostructures. As previously mentioned, 2D layered materials have been widely investigated either by fabricating bulk/thin film or by manufacturing hybrid composites as fillers. Moreover, studying the thermoelectric properties of single- or fewlayer materials via the fabrication of micro-nanodevices is another important field in the thermoelectric research environment. The thermoelectric properties of the bulk and of individual single-layer materials may differ and this may provide a deeper understanding of the physical mechanism behind their thermoelectric effect. For example, Kumar et al. [149] simulated the thermoelectric properties of bulk and monolayer $\mathrm{MoSe}_{2}$ and $\mathrm{WSe}_{2}$ by using the first-principles calculations and the semiclassical Boltzmann transport theory. The calculation results proved that the electrical conductivity, the Seebeck coefficient, and the thermal conductivity of the monolayer materials are extremely different from the bulk ones. These results allow one to optimize the thermoelectric properties based on the unit materials.

Decoupling the Seebeck coefficient, the electrical conductivity, and the thermal conductivity is a challenging problem to solve. However, the different mean free paths of electrons and phonons provide the possibility to decouple the electrical properties and the thermal properties, which are governed by the Wiedemann-Franz law [228]. In detail, several kinds of low-dimensional nanostructure material systems may enable high-speed transmittance of electrons by impeding the propagation of acoustic phonons. As a result, these compounds exhibit a suppressed thermal conductivity, whereas their electrical conductivity remains almost identical [93, 228, 229]. When the size of the material is small enough to influence its band structure, the profile of the density of states (DOS) can evolve into sharp shapes at the band edges due to the quantum confinement effect of these nanomaterials [228]. This phenomenon may increase the Seebeck coefficient, since this is strongly related to the change rate of the DOS near the Fermi energy [93, 230].

The typical structure of the micro-nanodevices, which are used to measure the thermoelectric performance of a singleor multilayer 2D material, is shown in Fig. 14. Two main micro-devices are currently used: a typical micro-device for the Seebeck coefficient and the electrical conductivity measurements and a suspended micro-device to determine the ZT value. The thermoelectric properties of nanostructure materials with different morphologies, including nanowires, nanosheets, nanoplates, and single- or multilayer 2D materials, can be measured via these micro-devices.

In a typical platform, the micro-device is usually patterned onto a silicon substrate which is coated with an insulating $\mathrm{SiO}_{2}$ layer. As shown in Fig. 14, one or two heaters are employed to build a temperature gradient through the nanosample. The samples are usually placed onto the substrate via a simple drop-casting process from a solution, which contains a suspension. Then, the metal electrodes are deposited onto the sample via an electron beam lithography process. The $\mathrm{Au} / \mathrm{Cr}$ metal contact with the sample is fabricated and serves 

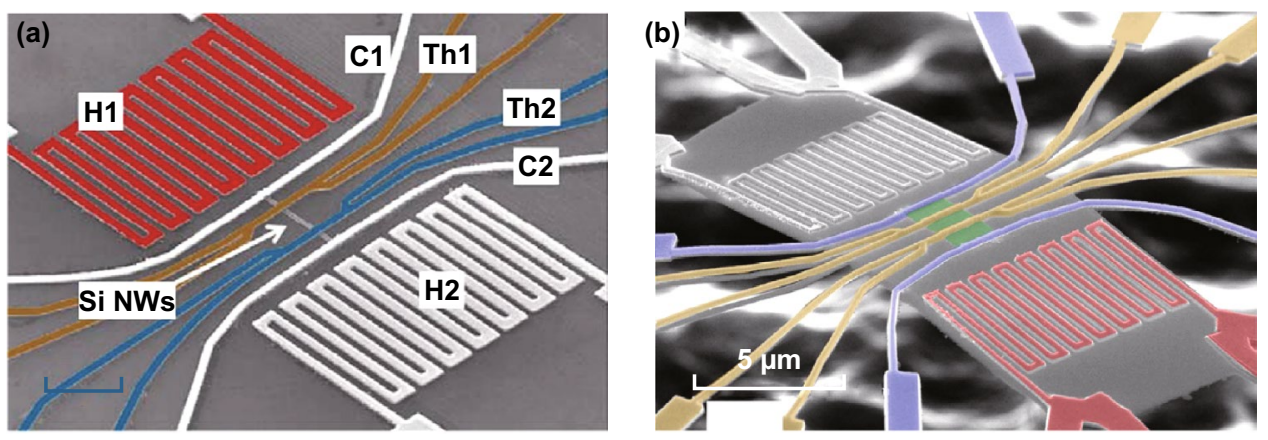

Fig. 14 SEM images of a non-suspended thermoelectric micro-device platform and $\mathbf{b}$ suspended thermoelectric micro-device platform. Reproduced with permission from Ref. [235]. Copyright 2008, Springer Nature

simultaneously as both an electrode and a thermometer [231]. However, for such micro-devices, since the electrodes and the sample are directly in contact with the substrate, only the Seebeck coefficient and the electrical conductivity can be measured. In order to evaluate the ZT value of the nanostructure 2D materials, a modification of this micro-device has been developed. As shown in Fig. 14b, the electrode and the micro-heaters are supported by five slender $\operatorname{SiN}_{x}$ beams which support the platform in vacuum onto the substrate. This micro-device platform allows one to measure the thermal conductivity of the nanomaterials and to calculate their ZT value [49, 232-235]. Under high vacuum, the heat loss of the sample via convection and radiation is negligible. Moreover, the monolithic silicon device minimizes the thermal contact resistances. When a micro-heater is applied to the system, the suspended micro-device in vacuum forces a heat flow across the samples. By carefully measuring the temperature at both sides of the sample and calculating the total amount of heat delivered to the micro-heater, the thermal conductance of the nanostructure 2D materials can be calculated. When combined with the dimensional data of the 2D materials, the thermal conductivity of each 2D material can be obtained based on such well-established thermometric technique [227, 231, 236].

A number of researchers have investigated the thermoelectric properties of individual 2D nanostructure materials for several years. In 2008, two remarkably high ZT values of 0.6 and 1.2 for two samples of highly doped silicon singlecrystalline nanowires at near room temperature have been reported independently by Heath and Yang's group [235, 237]. According to their studies, a suppression of the thermal conductivity was observed due to the rough surface and the reduction of diameter of the sample. By using these microstructure measurement platforms, the thermoelectric properties of many individual nanowires, nanosheets, quantum dot superlattices, and thin films were investigated [227, 231, 233, 234, 236, 238-245]. Recently, the thermoelectric properties of single-layer and multilayer 2D materials have been studied via these microstructure measurement platforms.

As previously mentioned, graphene and $\mathrm{BP}$ are considered potential high-performance thermoelectric materials and have been widely employed as composite fillers to enhance the thermoelectric properties of the composites. However, the thermoelectric properties of individual sample of graphene and BP have also been studied via these microstructure measurement platforms. Choi et al. [131] measured the thermoelectric performance at various temperatures and at gate electrical fields of BP and then studied its transport characteristics in samples with a thickness of 10-30 nm. The structure of this device is shown in Fig. 15a. The test results prove that the 2D Mott's variable rang hopping is a dominant mechanism in the thermal and electrical transport in thin BP samples. Saito et al. [134] investigated the gate-tuned thermoelectric performance of BP at low temperature $(210 \mathrm{~K})$. By using the electric-double-layer transistor configuration, the Seebeck coefficient of ion-gated BP reached $+510 \mu \mathrm{V} \mathrm{K}{ }^{-1}$ at $210 \mathrm{~K}$. This value is about 1.5 times higher than the value $\left(+340 \mu \mathrm{V} \mathrm{K}^{-1}\right.$ at $\left.300 \mathrm{~K}\right)$ of a bulk single crystal.

Several computational simulation results have shown that an ultrahigh thermoelectric performance can be achieved in various graphene nanostructures, including zigzag and armchair graphene nanoribbons, graphene nanomeshes, and graphene superlattices [48-50, 52-55, $64,246,247]$. These simulation results show that the ZT 

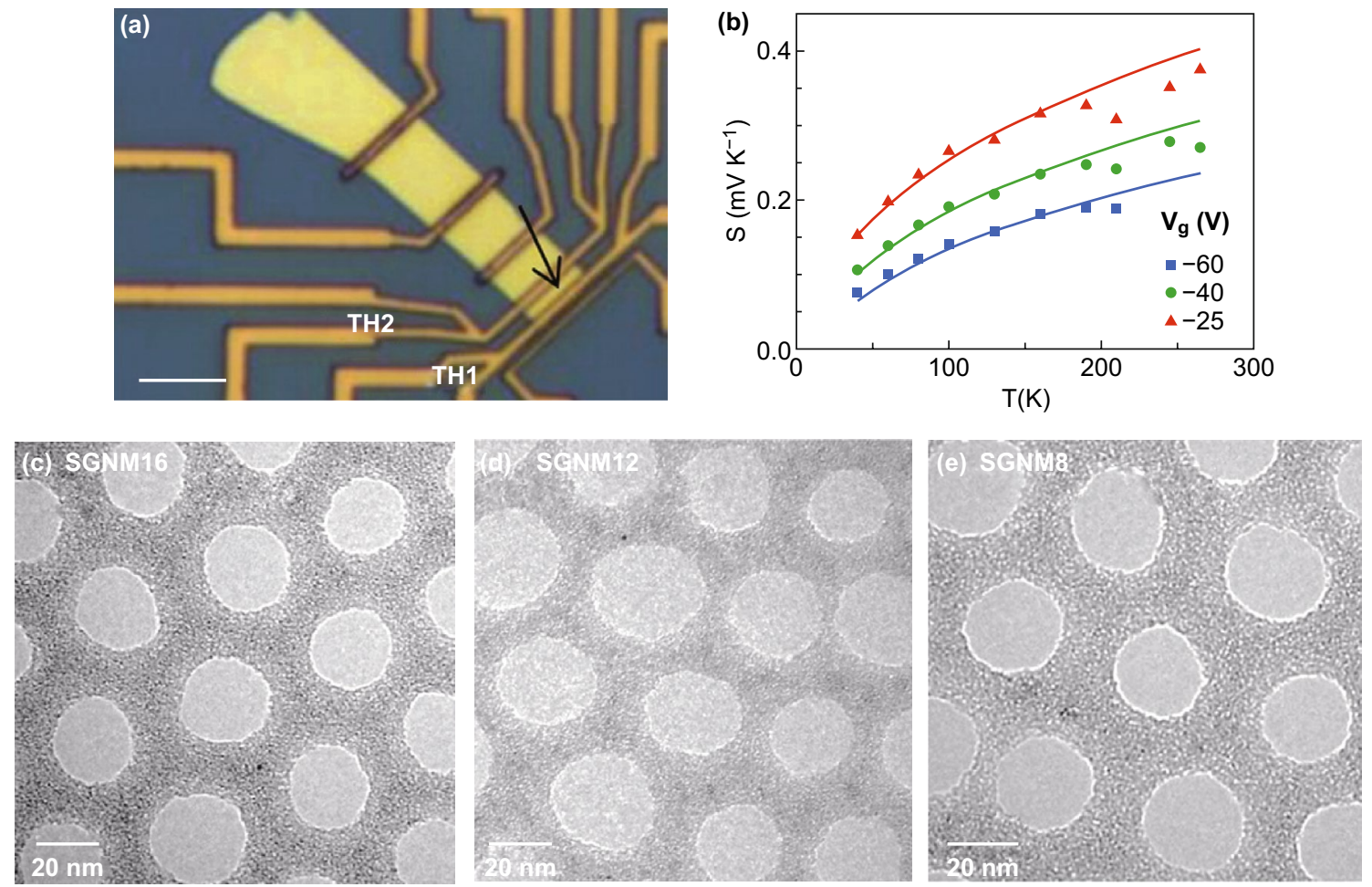

Fig. 15 a Optical microscope images of the measurement devices prepared onto a 300-nm-thick $\mathrm{SiO}_{2} / \mathrm{Si}$ substrates. b Seebeck coefficient of few-layer black phosphorus depending on temperature and gate voltage. Reproduced with permission from Ref. [131]. Copyright 2016, American Chemical Society. c TEM image of single-layer graphene nanomeshes with neck width of $16 \mathrm{~nm}$. TEM image of bilayer graphene nanomeshes with neck width of $\mathbf{d} 14 \mathrm{~nm}$ and $\mathbf{e} 8 \mathrm{~nm}$. Reproduced with permission from Ref. [249]. Copyright 2017, Elsevier

value can be enhanced by controlling the nanostructure or by employing a proper post-treatment. By controlling the microstructure and the layers of graphene, its thermoelectric efficiency can be greatly improved. Furthermore, by tuning the band structures, a high thermopower and a lower thermal conductivity can be achieved simultaneously due to the dominant effect of the phonon-edge scattering. In addition, these theoretic predictions have been proved via experimental measurements. Xiao et al. [248] found that the Seebeck coefficient of a few-layer graphene sample can be greatly enhanced $\left(>700 \mu \mathrm{V} \mathrm{K} \mathrm{K}^{-1}\right)$ after oxygen plasma treatment. Since a graphene monolayer is easy to damage during the plasma treatment process, the oxygen plasma treatment is not suitable. Oh et al. [249] fabricated singleand bilayer graphene nanomeshes with various neck widths via block copolymer self-assembly on graphene, as shown in Fig. 15c-e. Since the patterned graphene nanostructure can induce a dominant phonon-edge scattering and quantum confinements to control the electron and phonon-transport behavior, the bilayer graphene nanomeshes with $8 \mathrm{~nm}$ neck widths exhibit an ultralow thermal conductivity and an enhanced ZT value. Anno et al. achieved a ZT value of graphene, which is about 3 times higher than in the case of pristine graphene via defect engineering [66].

Besides graphene and BP, the thermoelectric properties of other 2D materials including 2D tellurium, TMDCs, and group IV-VI compounds were measured via these microstructure measurement platforms. Moreover, the nanopattern effect and the pattern geometry effect on the thermal and the thermoelectric phenomena of nanopatterned 2D materials were characterized [249]. For example, Qiu et al. [228] investigated the thermoelectric properties of 2D tellurium for the first time and reported a ZT value of 0.63 at room temperature. The Seebeck coefficient and the electrical conductivity of 2D Tellurium were measured by using the microstructure measurement platforms as shown in Fig. 14a. The thermal conductivity was measured via the micro-Raman method. Pettes et al. [234] investigated

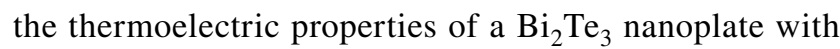
the thickness in the $9-25 \mathrm{~nm}$ range by using a suspended 
micro-device. When the thickness increases, the $\mathrm{Bi}_{2} \mathrm{Te}_{3}$ nanoplate exhibits a suppressed Seebeck coefficient, whereas both the electrical conductivity and the thermal conductivity are decreased. However, this result is mostly related to the surface band bending and the diffuse surface scattering of the electrons and the phonons in the nanoplates. The thermoelectric signature of 2D materials, such as the $\mathrm{WSe}_{2}$ single crystal, the single-layer $\mathrm{SnSe}$, the SnTe nanoplate, and the $\mathrm{Cd}_{3} \mathrm{As}_{2}$ superlattice, was also studied by using the microstructure measurement platform [243, 244, 250, 251].

As previously shown, the thermoelectric properties of individual 2D materials were investigated along the plane direction. As is well known, 2D materials usually exhibit a high anisotropy along the perpendicular direction and in-plane direction. However, it is difficult to measure the thermal conductivity, the Seebeck coefficient, and the electrical conductivity of single-layer and multilayer 2D materials along the perpendicular direction. Recently, several researchers have fabricated cross-plane thermoelectric devices to investigate the cross-plane thermoelectric properties of 2D materials. Figure 16a, $b$ shows an optical image of a completed device and the schematic diagram of its fabrication process. To manufacture such cross-plane device, a bottom/top metal 4-probe resistance temperature detector and a serpentine metal heater are patterned onto the silicon oxide/silicon substrate via electron beam lithography. This process is then followed by metal deposition of a metal electrode. Before the top resistance temperature detector is deposited, the sample should be capped with an insulating film of $\mathrm{Al}_{2} \mathrm{O}_{3}$. By using such microstructure measure platform, the Cronin's group studied the thermoelectric properties of $\mathrm{SnSe}_{2}$ and $\left(\mathrm{SnS}(\mathrm{e})_{n}\left(\mathrm{TiS}(\mathrm{e})_{n}\right.\right.$ $(n=1,3,4,5)$ thin-film layer materials along cross-plane direction $[252,253]$. The Seebeck coefficient and the thermal conductivity of the layered structure materials are strongly dependent on the number of layers. Moreover, several researchers found that the Seebeck coefficient, the electrical

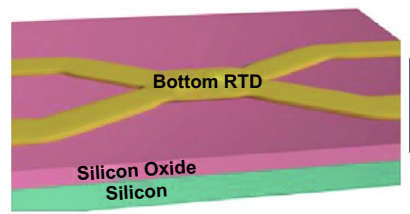

(a)
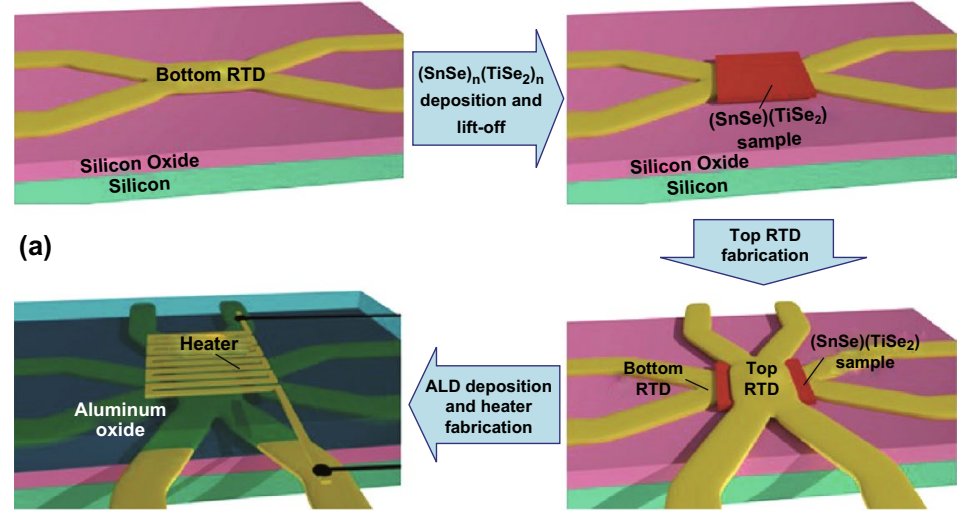
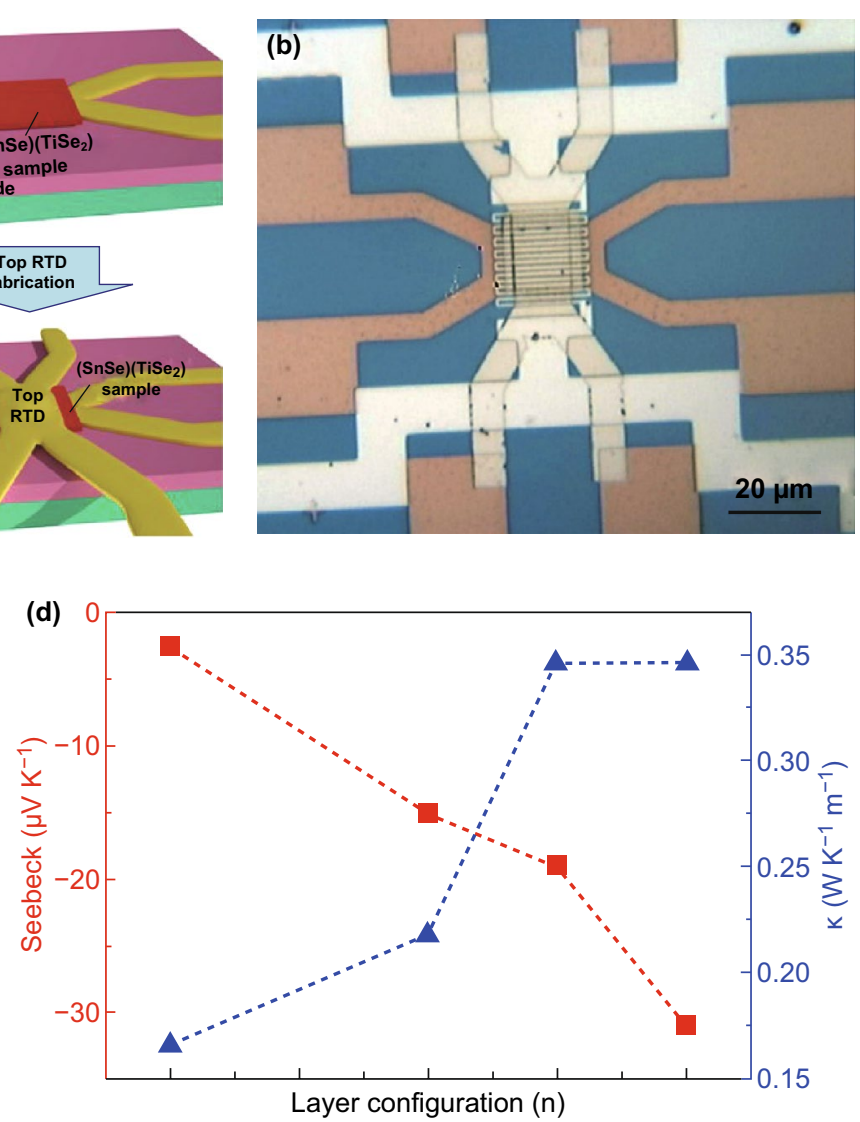

Fig. 16 a Schematic diagram of the device fabrication process, and $\mathbf{b}$ its optical microscope image. $\mathbf{c}$ STEM images of the $\left(\mathrm{SnSe}_{n}\left(\mathrm{TiSe}_{2}\right)_{n}\right.$ thin film $(n=1)$. d Cross-plane Seebeck coefficient and thermal conductivity of the $(\mathrm{SnSe})_{n}\left(\mathrm{TiSe}_{2}\right)_{n}$ thin film with different layer configurations. Reproduced with permission from Ref. [252]. Copyright 2017, American Chemical Society 
conductivity, and the thermal conductivity along cross-plane direction are very different from the properties along the inplane direction. This is in agreement with the properties of bulk thermoelectric materials based on 2D materials (such as $\mathrm{SnSe}, \mathrm{Bi}_{2} \mathrm{Te}_{3}$, and $\mathrm{Sb}_{2} \mathrm{Te}_{3}$ ). For example, $\mathrm{Li}$ et al. found that when the number of layers decreases from 5 to 1 , the cross-plane Seebeck coefficient decreases from -31 to $-2.5 \mu \mathrm{V} \mathrm{K}^{-1}$, whereas the cross-plane thermal conductivity decreases from 0.35 to $0.15 \mathrm{~W} \mathrm{mK}^{-1}$ (Fig. 16c, d), due to an increased interfacial phonon scattering [252]. Juang et al. [254] proposed a cross-plane micro-device onto a silicon substrate to measure the thermoelectric properties of the vertical graphene/gold nanoparticles heterostructure along the perpendicular direction. Since the Au nanoparticles can further inhibit the phonon transport and enhance the electrical conductivity along the perpendicular direction, a high ZT value larger than 1.0 (at room temperature) can be achieved for a single-layer graphene. Chen et al. [255] fabricated a graphene/hexagonal boron nitride/graphene heterostructure device and measured its thermoelectric transport properties. The top and bottom graphene surfaces in this device work as both an electrode and a micro-heater to induce a temperature gradient. The measured thermoelectric properties are useful to understand the thermoelectric component in the cross-plane behavior of emerging $2 \mathrm{D}$ heterostructure devices.

The development of micro-nanoprocessing techniques enables us to fabricate micro-devices to investigate the thermoelectric properties of individual 2D nanostructure materials. The study of the thermoelectric transport characteristics of individual 2D materials is pivotal to understand its physical mechanism. The massive proliferation of 2D materials, such as TMDCs, BP, MXenes, graphene, and Xene, offers new opportunities to engineer such compounds and to fabricate high-performance thermoelectric devices. The device structures reviewed in this section serve as a general approach to characterize the in-plane and cross-plane transport properties of 2D materials.

\section{Thermoelectric Materials Combined with Photodetection}

Thermoelectric materials have been widely used as power generators, cooling devices, and sensors. Recently, they have been found to work as power supplies and can be used to develop highly efficient and wearable self-powered electronics devices. As a group of developing materials, 2D materials have been widely employed as optoelectronic devices, including in the manufacturing of photodetectors and photovoltaic devices. When irradiated by light, these devices convert photons into electric current due to separation of the excited electron-hole pair via a built-in electric field. The development of low-cost and high-performance broadband photodetectors is the key to fulfill different application requirements. The photothermoelectric effect, which is based on the Seebeck effect, enables the device to generate a photocurrent due to the temperature gradient induced by the absorbed light on an electric voltage. Moreover, this temperature gradient would be generated across the materials due to light irradiation and the photocurrent is generated by the photothermoelectric effect. Self-power photodetectors are promising devices, which can be employed in a large variety of application including sensing, environmental monitoring, night vision, and astronomy.

2D materials, such as graphene and BP, have been widely used as photothermal agents due to their efficient photothermal conversion efficient [122, 256-259]. Gabor found that upon heating a junction consisting of a single graphene sheet by shining laser onto it, a thermoelectric voltage is generated across the junction [260]. However, this kind of photothermoelectric effect was found in other 2D materials system, as well [51, 71, 126, 261-264]. As predicted by Basko, this effect can be potentially exploited in novel optoelectronic devices [70].

In 2011, Kraemer et al. [265] reported a novel solar thermal flat panel, which converts thermal energy into electric power based on the Seebeck effect and on the high thermal concentration. The peak efficiency of such solar thermoelectric generator is close to that of a common solar cell (4.6\%). However, the total power conversion efficiency of this device is limited by the photothermal and thermoelectric efficiency [56, 265].

Besides their use as solar energy converters, 2D thermoelectric materials also can be employed in the fabrication of high-performance self-power photodetectors. When compared to other photodetection mechanisms, such as photoconduction, photovoltage, and bolometry, the photothermoelectric effect achieves a broadband detection without an external bias at room temperature. A photodetector based on the photothermoelectric effect mechanism exhibits a responsivity, which is related to the light-induced temperature 
gradient and to its Seebeck coefficient. The temperature gradient is determined by the absorption, heat capacity, and the photothermal conversion efficiency of the material.

Graphene and BP are the most widely studied 2D materials and have attracted a large attention. In 2010, $\mathrm{Xu}$ et al. [51] found that the photothermoelectric effect has a pronounced effect into the photocurrent generation process at the graphene interface in field-effect transistors. This phenomenon has several positive benefits in the design of high-performance optoelectronics devices based on graphene. As shown in Fig. 17a, Cai et al. [266] fabricated a simple thermoelectric terahertz photodetector based on graphene, which exhibits an excellent sensitivity exceeding $10 \mathrm{~V} \mathrm{~W}^{-1}\left(700 \mathrm{~V} \mathrm{~W}^{-1}\right)$ at room temperature and noise-equivalent power less than $1100 \mathrm{pW} \mathrm{Hz}^{-1 / 2}$
(20 $\mathrm{pW} \mathrm{Hz}^{-1 / 2}$ ), referenced to the incident (absorbed) power. The performance of such graphene-based thermoelectric terahertz photodetectors can compete with the best room-temperature terahertz detectors to develop optimally coupled devices. Echtermeyer et al. [61] investigated the influence of the wavelength on the performance of metalgraphene-metal photodetectors via polarization-dependent measurements. The device that used in this study is shown in Fig. 17b. As shown in Fig. 17c, Muench et al. report a compact, photothermoelectric-based, waveguide- integrated, plasmonic enhanced graphene photodetector for telecom wavelengths operating at zero dark current. Owing to the voltage generated by the photothermoelectric, the graphene photodetector exhibits an external responsivity $\sim 12.2 \mathrm{~V} \mathrm{~W}^{-1}$ and a $3 \mathrm{~dB}$ bandwidth $\sim 42 \mathrm{GHz}$ [267].
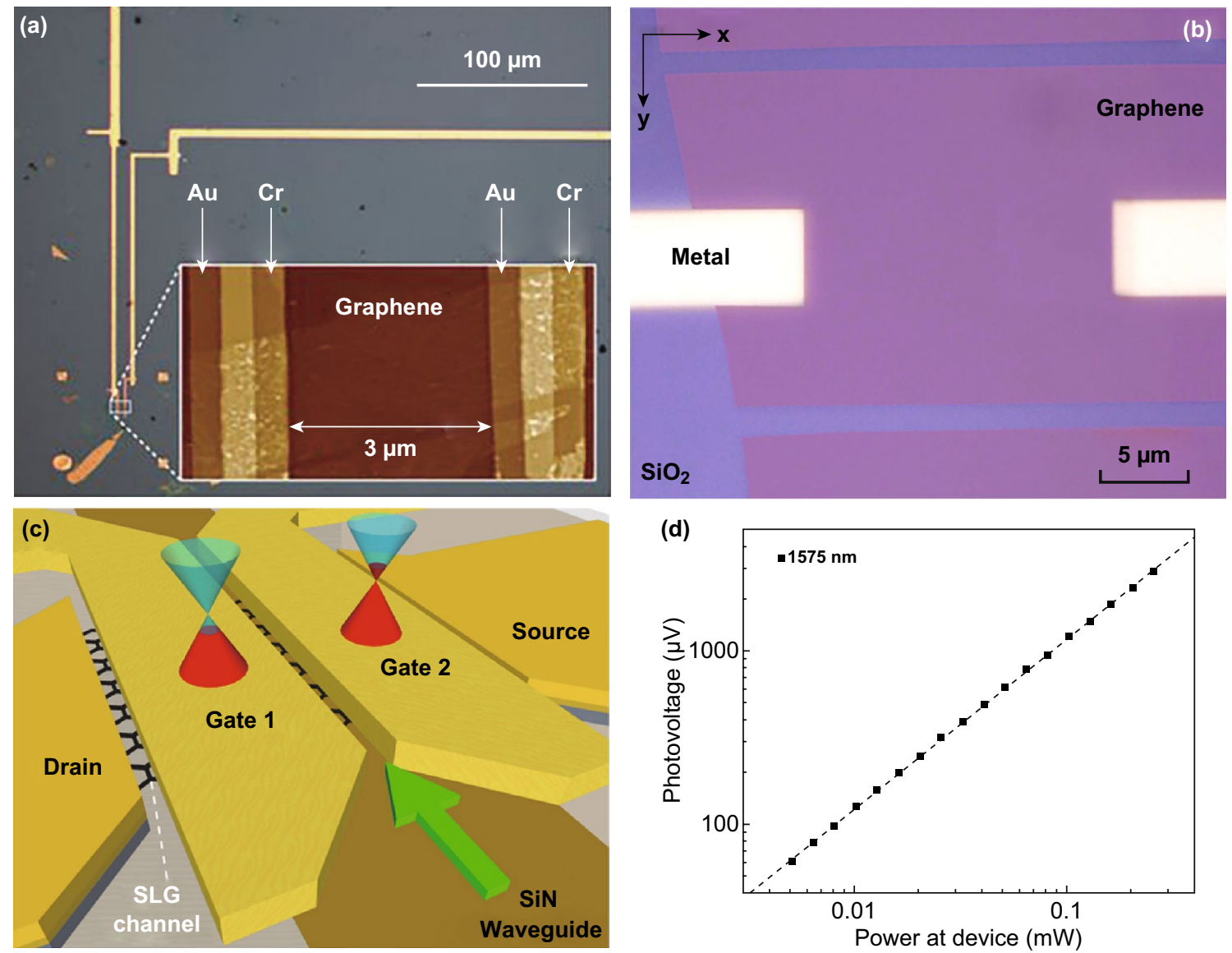

Fig. 17 a Optical micrograph and atomic force microscopy of graphene photodetector with asymmetric metal electrodes. Reproduced with permission from Ref. [266]. Copyright 2014, Springer Nature. b Optical micrograph of the graphene-based photodetector devices. Reproduced with permission from Ref. [61]. Copyright 2014, American Chemical Society. c Scheme of the single-layer graphene photodetector SiN waveguide. The green arrow indicates the light propagation direction. d The photovoltage $V_{\mathrm{ph}}$ dependence of optical power. Reproduced with permission from Ref. [267]. Copyright 2019, American Chemical Society 
The photovoltage $\left(V_{\mathrm{ph}}\right)$ generated by the thermoelectric current as for the Seebeck effect dependence on power is shown in Fig. 17d. The linear response indicates a powerindependent external voltage responsivities in the tested optical power range [267].

As graphene, BP has also been studied as a high-performance photothermoelectric photodetector. Hong et al. [126] investigated the electrical transport and optoelectronic properties of FETs manufactured from a few-layer BP sample. Their results reveal that the photocurrent signals at the BP-electrode junctions can be mainly attributed to the photovoltaic effect in its the off-state, whereas the photothermoelectric effect occurs during its on-state. Leong et al. [268] fabricated a device based on exfoliated BP nanoflakes with a responsivity of $1 \mathrm{mV} \mathrm{W}^{-1}$ for a $2.5-\mathrm{THz}$ beam with a diameter of $200 \mu \mathrm{m}$. In this case, the photothermoelectric effect was found to be the primary source of the $\mathrm{THz}$ photosignal.

The photothermoelectric effect of several 2D TMDC materials, such as $\mathrm{MoS}_{2}, \mathrm{WSe}_{2}$, and $\mathrm{Bi}_{2} \mathrm{Se}_{3}$, has also been widely investigated [71, 261, 262, 264]. Buscema et al. [71] studied the photoresponse of $\mathrm{MoS}_{2}$ monolayer FETs via scanning photocurrent microscopy. The device and results are shown in Fig. 18. To summarize, the photocurrent generation in a $\mathrm{MoS}_{2}$ monolayer is dominated by the photothermoelectric effect. The separation of the photoexcited electron-hole pairs across the Schottky barriers at the $\mathrm{MoS}_{2} /$ electrode interfaces only plays a marginal role. As shown in Fig. 18h, a large and controllable Seebeck coefficient in the $-1 \times 10^{5}$ to $-4 \times 10^{2} \mu \mathrm{V} \mathrm{K}^{-1}$ range was observed in the case of $\mathrm{MoS}_{2}$ monolayer. However, the mechanism behind the generation of the photocurrent is different when compared to other 2D TMDC materials. In order to deeply understand such physical mechanism, Groenendijk et al. [262] studied the photocurrent in a $\mathrm{WSe}_{2}$ sample by fabricating a doublegated $\mathrm{WSe}_{2}$ device and by applying varied gate voltages and illumination power. The results show that the photocurrent of the $\mathrm{WSe}_{2}$-based devices (for both the PN and NP configurations) is mainly generated by the photovoltaic effect. Moreover, a maximum responsivity of $0.7 \mathrm{~mA} \mathrm{~W}^{-1}$ at 532-nm illumination was obtained. In the PP configurations devices, the photocurrent mainly is generated by the photothermoelectric effect and the intensity is about 2 times larger than that generated by the photovoltaic effect. Due to the strong optical absorption caused by an asymmetry in flake thickness or by the optical absorption of the electrodes, a sizable temperature gradient can be generated across the
2D material-based devices. In addition, the photothermoelectric effect plays a significant role in such devices and can be used to develop high-performance self-power optoelectronic devices.

Photothermoelectric photodetectors have exhibited their advantages in developing low-cost, uncooled ultrafast and ultrabroadband photodetectors, and their performance has been significantly enhanced owing to the development of low-dimensional materials and the micro-nanofabrication technology. However, due to the limited effective active surface area, the saturation effect and relatively low practical Seebeck coefficient at room temperature limit the temperature gradient in the case of exfoliated or single-crystal 2D materials. For this reason, no high voltage can be generated. Although tremendous progress has been made in recent years, more work needs to be done to further optimize the performance of $2 \mathrm{D}$ material-based photothermoelectric photodetectors.

\section{Conclusions and Outlook}

During the last decades, a significant progress has been achieved in the development of thermoelectric materials. As new members of the thermoelectric material family, 2D materials have attracted a large attention and many milestone investigations have been reported. Due to their unique electronic, thermal, and mechanic properties, 2D thermoelectric materials are expected to be the next-generation high-performance thermoelectric materials.

In this review, the thermoelectric properties of various 2D materials, such as TMDCs, graphene, BP, group IVA-VA compounds, MXene, and their nanocomposites, were illustrated in detail. Moreover, the state-of-the-art of theoretical and experimental data were presented to elucidate the relation among various factors which determine the thermoelectric properties of these materials. Although a tremendous progress has been achieved in the past few years, these properties still need to be improved for their practical application as thermoelectric devices. Here, some major outlooks are presented to address this issue:

1. The thermoelectric performance of $2 \mathrm{D}$ materials is still much lower then for conventional bulk thermoelectric materials. A precise chemical doping during the fabrication and the functionalization of these composites must 
(a)

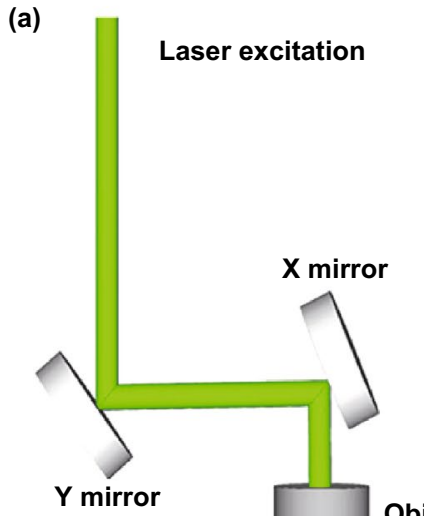

(b)

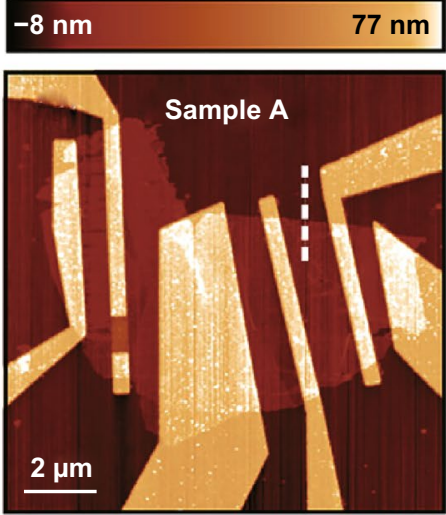

(c)

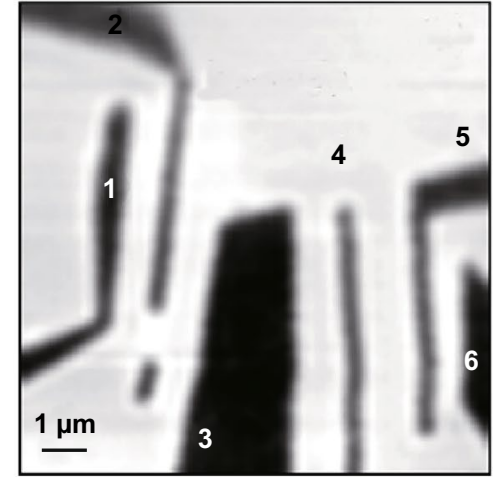

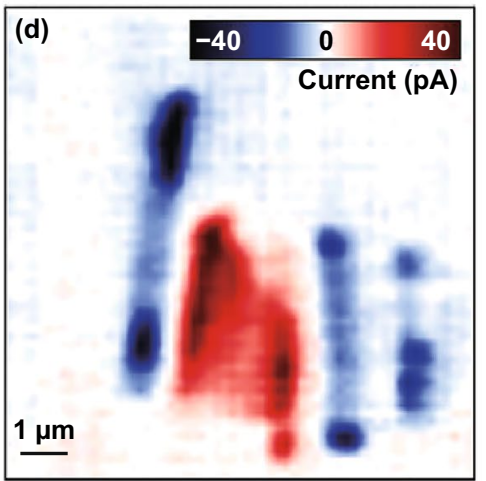

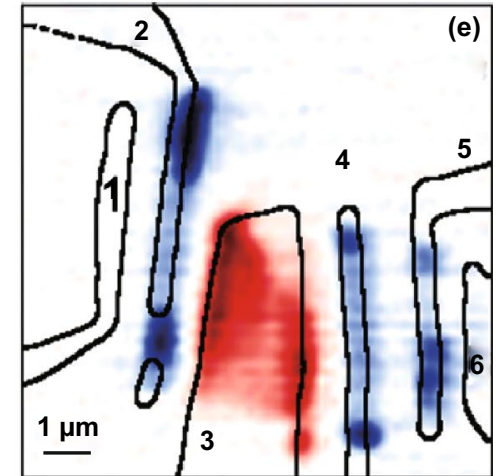

(h) 10
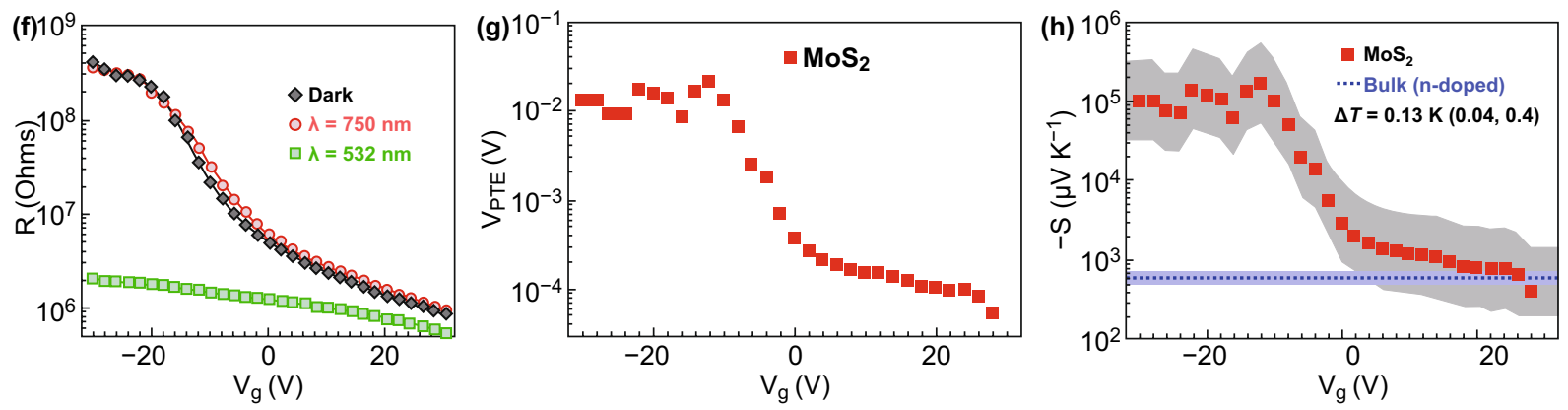

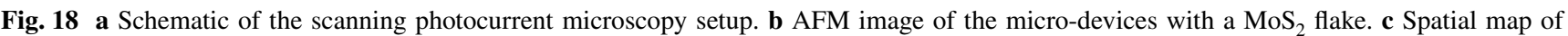
the intensity of the reflected light from the device. d Photocurrent image of the $\mathrm{MoS}_{2}$ FET. e Superposition of the photocurrent map and the contours of the electrodes, which were obtained from the light reflection map. $\mathbf{f}$ Resistance and $\mathbf{g}$ photothermoelectric voltage of the MoS $\mathrm{S}_{2}$ device as a function of the gate voltage in its dark state and with the laser spot placed on the $\mathrm{MoS}_{2} /$ electrode interface. Estimated Seebeck coefficient as a function of the gate voltage. Reproduced with permission from Ref. [71]. Copyright 2013, American Chemical Society

be performed to achieve a high value of ZT value. This remains a challenging issue.

2. The electronic and phonon-transport mechanisms of $2 \mathrm{D}$ materials are still obscure. Although the power factors of individual single- and few-layer 2D materials have been investigated and a series of possible mechanisms have been proposed, it is still impossible to decouple these factors. The current knowledge of the effects of the interfacial electronic and the phonon-transport mecha- nism on the thermoelectric performance is still limited. Their deeper understanding should guide the design of novel thermoelectric composite and further improve their thermoelectric properties.

3. The thermal conductivity of 2D materials is too high, and this limits their thermoelectric performance. The inherent thermal conductivity of the most common 2D materials, including graphene, TMDCs, and BP, is much higher than for the traditional semiconductor thermo- 
electric materials. Their structural modifications, as well as the introduction of structural defects and the control of their elemental components, are likely to have a positive effect on the thermal conductivity of these materials.

4. The fabrication realization and the characterization of 2D material-based thin films are still challenging. With the increase in the demand of wearable and portable flexible devices, thin-film thermoelectric materials have received a wide attention. Although they have been studied for very short time when compared to bulk thermoelectric materials, they are considered the next generation of thermoelectric materials for practical applications. Due to their excellent electronic and mechanical properties, several researchers have tried to fabricate thin-film thermoelectric materials by using 2D nanomaterials and their composites. However, the measurements of the thermal conductivity of thin films are still challenging.

5. It is of pivotal importance to achieve a facile production of high-quality and scalable 2D material. This is currently an active research topic. Until now, many methods have been employed to fabricate 2D materials, such as chemical vapor deposition, mechanical exfoliation, atomic layer deposition, molecular beam epitaxy, physical vapor deposition liquid exfoliation, and mechanical exfoliation. However, it is still challenging to manufacture such materials with a controlled structure via scaleup methods.

Acknowledgements This work was supported by National Science Foundation for Young Scientists of China (No. 61905161 and 51702219), the National Natural Science Foundation of China (No. 61975134, 61875138 and 61775147), the Science and Technology Innovation Commission of Shenzhen (No. JCYJ20180206121837007), and the Shenzhen Nanshan District Pilotage Team Program (LHTD20170006).

Open Access This article is licensed under a Creative Commons Attribution 4.0 International License, which permits use, sharing, adaptation, distribution and reproduction in any medium or format, as long as you give appropriate credit to the original author(s) and the source, provide a link to the Creative Commons licence, and indicate if changes were made. The images or other third party material in this article are included in the article's Creative Commons licence, unless indicated otherwise in a credit line to the material. If material is not included in the article's Creative Commons licence and your intended use is not permitted by statutory regulation or exceeds the permitted use, you will need to obtain permission directly from the copyright holder. To view a copy of this licence, visit http://creativecommons.org/ licenses/by/4.0/.

\section{References}

1. Z.G. Chen, G. Han, L. Yang, L. Cheng, J. Zou, Nanostructured thermoelectric materials: current research and future challenge. Prog. Nat. Sci. 22(6), 535-549 (2012). https://doi. org/10.1016/j.pnsc.2012.11.011

2. T. Zhu, Y. Liu, C. Fu, J.P. Heremans, J.G. Snyder, X. Zhao, Compromise and synergy in high-efficiency thermoelectric materials. Adv. Mater. 29(14), 1605884 (2017). https://doi. org/10.1002/adma.201605884

3. G.J. Snyder, E.S. Toberer, Complex thermoelectric materials. Nat. Mater. 7(2), 105-114 (2008). https://doi.org/10.1038/nmat2090

4. M. Rull-Bravo, A. Moure, J.F. Fernandez, M. MartinGonzalez, Skutterudites as thermoelectric materials: revisited. RSC Adv. 5(52), 41653-41667 (2015). https:// doi.org/10.1039/c5ra03942h

5. P. Sundarraj, D. Maity, S.S. Roy, R.A. Taylor, Recent advances in thermoelectric materials and solar thermoelectric generators - a critical review. RSC Adv. 4(87), 46860-46874 (2014). https://doi.org/10.1039/c4ra05322b

6. G. Tan, L.-D. Zhao, M.G. Kanatzidis, Rationally designing high-performance bulk thermoelectric materials. Chem. Rev. 116(19), 12123-12149 (2016). https://doi. org/10.1021/acs.chemrev.6b00255

7. C. Gayner, K.K. Kar, Recent advances in thermoelectric materials. Prog. Mater Sci. 83, 330-382 (2016). https://doi. org/10.1016/j.pmatsci.2016.07.002

8. G. Zhang, Y.-W. Zhang, Thermoelectric properties of twodimensional transition metal dichalcogenides. J. Mater. Chem. C 5(31), 7684-7698 (2017). https://doi.org/10.1039/ c7tc01088e

9. D. Li, C. Luo, Y. Chen, D. Feng, Y. Gong, C. Pan, J. He, High performance polymer thermoelectric composite achieved by carbon-coated carbon nanotubes network. ACS Appl. Energy Mater. 2(4), 2427-2434 (2019). https://doi. org/10.1021/acsaem.9b00334

10. Z.-H. Ge, L.-D. Zhao, D. Wu, X. Liu, B.-P. Zhang, J.-F. $\mathrm{Li}, \mathrm{J}$. He, Low-cost, abundant binary sulfides as promising thermoelectric materials. Mater. Today 19(4), 227-239 (2016). https://doi.org/10.1016/j.mattod.2015.10.004

11. S. Ortega, M. Ibanez, Y. Liu, Y. Zhang, M.V. Kovalenko, D. Cadavid, A. Cabot, Bottom-up engineering of thermoelectric nanomaterials and devices from solution-processed nanoparticle building blocks. Chem. Soc. Rev. 46(12), 3510-3528 (2017). https://doi.org/10.1039/c6cs00567e

12. R. Kroon, D.A. Mengistie, D. Kiefer, J. Hynynen, J.D. Ryan, L. Yu, C. Muller, Thermoelectric plastics: from design to synthesis, processing and structure-property relationships. Chem. Soc. Rev. 45(22), 6147-6164 (2016). https://doi.org/10.1039/c6cs00149a

13. L. Wang, Z. Zhang, L. Geng, T. Yuan, Y. Liu et al., Solution-printable fullerene/TiS $\mathrm{S}_{2}$ organic/inorganic hybrids for high-performance flexible $n$-type thermoelectrics. Energy Environ. Sci. 11(5), 1307-1317 (2018). https://doi. org/10.1039/c7ee03617e 
14. J. Yang, H.-L. Yip, A.K.Y. Jen, Rational design of advanced thermoelectric materials. Adv. Energy Mater. 3(5), 549565 (2013). https://doi.org/10.1002/aenm.201200514

15. J.R. Sootsman, D.Y. Chung, M.G. Kanatzidis, New and old concepts in thermoelectric materials. Angew. Chem. Int. Ed. 48(46), 8616-8639 (2009). https://doi.org/10.1002/ anie. 200900598

16. J.P. Heremans, V. Jovovic, E.S. Toberer, A. Saramat, K. Kurosaki, A. Charoenphakdee, S. Yamanaka, G.J. Snyder, Enhancement of thermoelectric efficiency in $\mathrm{PbTe}$ by distortion of the electronic density of states. Science 321(5888), 554-557 (2008). https://doi.org/10.1126/scien ce. 1159725

17. B. Poudel, Q. Hao, Y. Ma, Y. Lan, A. Minnich et al., Highthermoelectric performance of nanostructured bismuth antimony telluride bulk alloys. Science 320(5876), 634638 (2008). https://doi.org/10.1126/science.1156446

18. K. Biswas, J. He, I.D. Blum, C.-I. Wu, T.P. Hogan, D.N. Seidman, V.P. Dravid, M.G. Kanatzidis, High-performance bulk thermoelectrics with all-scale hierarchical architectures. Nature 489(7416), 414-418 (2012). https://doi. org/10.1038/nature11439

19. Y. Pei, X. Shi, A. LaLonde, H. Wang, L. Chen, G.J. Snyder, Convergence of electronic bands for high performance bulk thermoelectrics. Nature 473(7345), 66-69 (2011). https:// doi.org/10.1038/nature09996

20. J.P. Heremans, C.M. Thrush, D.T. Morelli, Thermopower enhancement in lead telluride nanostructures. Phys. Rev. B 70(11), 115334 (2004). https://doi.org/10.1103/PhysR evB.70.115334

21. F. Schedin, A.K. Geim, S.V. Morozov, E.W. Hill, P. Blake, M.I. Katsnelson, K.S. Novoselov, Detection of individual gas molecules adsorbed on graphene. Nat. Mater. 6, 652655 (2007). https://doi.org/10.1038/nmat1967

22. S. Grimme, J. Antony, S. Ehrlich, H. Krieg, A consistent and accurate $a b$ initio parametrization of density functional dispersion correction (DFT-D) for the 94 elements H-Pu. J. Chem. Phys. 132(15), 154104 (2010). https://doi. org/10.1063/1.3382344

23. C. Nie, L. Yu, X. Wei, J. Shen, W. Lu, W. Chen, S. Feng, H. Shi, Ultrafast growth of large-area monolayer $\mathrm{MoS}_{2}$ film via gold foil assistant CVD for a highly sensitive photodetector. Nanotechnology 28(27), 275203 (2017). https://doi. org/10.1088/1361-6528/aa7473

24. Y. Yu, G.-H. Nam, Q. He, X.-J. Wu, K. Zhang et al., High phase-purity $1 \mathrm{~T}^{\prime}-\mathrm{MoS}_{2}$ - and $1 \mathrm{~T}^{\prime}-\mathrm{MoSe}_{2}$ - layered crystals. Nat. Chem. 10(6), 638-643 (2018). https://doi.org/10.1038/ s41557-018-0035-6

25. J. Lai, X. Liu, J. Ma, Q. Wang, K. Zhang et al., Anisotropic broadband photoresponse of layered type-II weyl semimetal $\mathrm{MoTe}_{2}$. Adv. Mater. 30(22), e1707152 (2018). https://doi. org/10.1002/adma.201707152

26. J. Yang, T. Lu, Y.W. Myint, J. Pei, D. Macdonald, J.-C. Zheng, Y. Lu, Robust excitons and trions in monolayer $\mathrm{MoTe}_{2}$. ACS Nano 9(6), 6603-6609 (2015). https://doi. org/10.1021/acsnano.5b02665
27. C. Lee, X. Wei, J.W. Kysar, J. Hone, Measurement of the elastic properties and intrinsic strength of monolayer graphene. Science 321(5887), 385-388 (2008). https://doi. org/10.1126/science.1157996

28. L. Li, Y. Yu, G.J. Ye, Q. Ge, X. Ou et al., Black phosphorus field-effect transistors. Nat. Nanotechnol. 9(5), 372-377 (2014). https://doi.org/10.1038/nnano.2014.35

29. M. Naguib, O. Mashtalir, J. Carle, V. Presser, J. Lu, L. Hultman, Y. Gogotsi, M.W. Barsoum, Two-dimensional transition metal carbides. ACS Nano 6(2), 1322-1331 (2012)

30. W. Tao, N. Kong, X. Ji, Y. Zhang, A. Sharma et al., Emerging two-dimensional monoelemental materials (Xenes) for biomedical applications. Chem. Soc. Rev. 48(11), 28912912 (2019). https://doi.org/10.1039/c8cs00823j

31. A. Molle, J. Goldberger, M. Houssa, Y. Xu, S.C. Zhang, D. Akinwande, Buckled two-dimensional Xene sheets. Nat. Mater. 16(2), 163-169 (2017). https://doi.org/10.1038/nmat4 802

32. Y. Liu, S. Zhang, J. He, Z.M. Wang, Z. Liu, Recent progress in the fabrication, properties, and devices of heterostructures based on 2D materials. Nano-Micro Lett. 11, 13 (2019). https ://doi.org/10.1007/s40820-019-0245-5

33. L.-D. Zhao, S.-H. Lo, Y. Zhang, H. Sun, G. Tan, C. Uher, C. Wolverton, V.P. Dravid, M.G. Kanatzidis, Ultralow thermal conductivity and high thermoelectric figure of merit in SnSe crystals. Nature 508(7496), 373-377 (2014). https://doi. org/10.1038/nature13184

34. C. Chang, M. Wu, D. He, Y. Pei, C.-F. Wu et al., 3D charge and $2 \mathrm{D}$ phonon transports leading to high out-of-plane in n-type SnSe crystals. Science 360(6390), 778 (2018). https ://doi.org/10.1126/science.aaq1479

35. K. Nielsch, J. Bachmann, J. Kimling, H. Boettner, Thermoelectric nanostructures: from physical model systems towards nanograined composites. Adv. Energy Mater. 1(5), 713-731 (2011). https://doi.org/10.1002/aenm.201100207

36. H. Wang, C. Li, P. Fang, Z. Zhang, J.Z. Zhang, Synthesis, properties, and optoelectronic applications of two-dimensional $\mathrm{MoS}_{2}$ and $\mathrm{MoS}_{2}$-based heterostructures. Chem. Soc. Rev. 47(16), 6101-6127 (2018). https://doi.org/10.1039/ c8cs00314a

37. F. Wang, Z. Wang, L. Yin, R. Cheng, J. Wang et al., 2D library beyond graphene and transition metal dichalcogenides: a focus on photodetection. Chem. Soc. Rev. 47(16), 6296-6341 (2018). https://doi.org/10.1039/c8cs00255j

38. S. Wang, A. Robertson, J.H. Warner, Atomic structure of defects and dopants in 2D layered transition metal dichalcogenides. Chem. Soc. Rev. 47(17), 6764-6794 (2018). https ://doi.org/10.1039/c8cs00236c

39. Y. Zhang, Y. Zheng, K. Rui, H.H. Hng, K. Hippalgaonkar et al., 2D black phosphorus for energy storage and thermoelectric applications. Small 13(28), 1700661 (2017). https:// doi.org/10.1002/smll.201700661

40. H. Babaei, J.M. Khodadadi, S. Sinha, Large theoretical thermoelectric power factor of suspended single-layer $\mathrm{MoS}_{2}$. 
Appl. Phys. Lett. 105(19), 193901 (2014). https://doi. org/10.1063/1.4901342

41. K.S. Novoselov, A.K. Geim, S.V. Morozov, D. Jiang, Y. Zhang, S.V. Dubonos, I.V. Grigorieva, A.A. Firsov, Electric field effect in atomically thin carbon films. Science 306(5696), 666-669 (2004). https://doi.org/10.1126/scien ce. 1102896

42. A.H. Castro Neto, F. Guinea, N.M.R. Peres, K.S. Novoselov, A.K. Geim, The electronic properties of graphene. Rev. Mod. Phys. 81(1), 109-162 (2009). https://doi.org/10.1103/RevMo dPhys.81.109

43. A. Dey, O.P. Bajpai, A.K. Sikder, S. Chattopadhyay, M.A.S. Khan, Recent advances in CNT/graphene based thermoelectric polymer nanocomposite: a proficient move towards waste energy harvesting. Renew. Sust. Energy Rev. 53, 653-671 (2016). https://doi.org/10.1016/j.rser.2015.09.004

44. K.I. Bolotin, K.J. Sikes, Z. Jiang, M. Klima, G. Fudenberg, J. Hone, P. Kim, H.L. Stormer, Ultrahigh electron mobility in suspended graphene. Solid State Commun. 146(9-10), 351-355 (2008). https://doi.org/10.1016/j.ssc.2008.02.024

45. P. Wei, W. Bao, Y. Pu, C.N. Lau, J. Shi, Anomalous thermoelectric transport of dirac particles in graphene. Phys. Rev. Lett. 102(16), 166808 (2009). https://doi.org/10.1103/PhysR evLett.102.166808

46. A.A. Balandin, S. Ghosh, W. Bao, I. Calizo, D. Teweldebrhan, F. Miao, C.N. Lau, Superior thermal conductivity of single-layer graphene. Nano Lett. 8(3), 902-907 (2008). https ://doi.org/10.1021/n10731872

47. D. Dragoman, M. Dragoman, Giant thermoelectric effect in graphene. Appl. Phys. Lett. 91(20), 203116 (2007). https:// doi.org/10.1063/1.2814080

48. Y. Chen, T. Jayasekera, A. Calzolari, K.W. Kim, M.B. Nardelli, Thermoelectric properties of graphene nanoribbons, junctions and superlattices. J. Phys. Condens. Mat. 22(37), 372202 (2010). https://doi.org/10.1088/0953$8984 / 22 / 37 / 372202$

49. S.-G. Nam, D.-K. Ki, H.-J. Lee, Thermoelectric transport of massive Dirac fermions in bilayer graphene. Phys. Rev. B 82(24), 245416 (2010). https://doi.org/10.1103/PhysR evB.82.245416

50. H. Sevincli, G. Cuniberti, Enhanced thermoelectric figure of merit in edge-disordered zigzag graphene nanoribbons. Phys. Rev. B 81(11), 113401 (2010). https://doi.org/10.1103/PhysR evB.81.113401

51. X. Xu, N.M. Gabor, J.S. Alden, A.M. van der Zande, P.L. McEuen, Photo-thermoelectric effect at a graphene interface junction. Nano Lett. 10(2), 562-566 (2010). https://doi. org/10.1021/n1903451y

52. T. Gunst, T. Markussen, A.-P. Jauho, M. Brandbyge, Thermoelectric properties of finite graphene antidot lattices. Phys. Rev. B 84(15), 155449 (2011). https://doi.org/10.1103/PhysR evB.84.155449

53. H. Karamitaheri, M. Pourfath, M. Pazoki, R. Faez, H. Kosina, Graphene-based antidots for thermoelectric applications. J.
Electrochem. Soc. 158(12), K213-K216 (2011). https://doi. org/10.1149/2.025112jes

54. F. Mazzamuto, V.H. Nguyen, Y. Apertet, C. Caer, C. Chassat, J. Saint-Martin, P. Dollfus, Enhanced thermoelectric properties in graphene nanoribbons by resonant tunneling of electrons. Phys. Rev. B 83(23), 235426 (2011). https://doi. org/10.1103/PhysRevB.83.235426

55. C.-R. Wang, W.-S. Lu, L. Hao, W.-L. Lee, T.-K. Lee, F. Lin, I.C. Cheng, J.-Z. Chen, Enhanced thermoelectric power in dual-gated bilayer graphene. Phys. Rev. Lett. 107(18), 186602 (2011). https://doi.org/10.1103/PhysRevLett.107.186602

56. Y. Chen, K. Chen, H. Bai, L. Li, Electrochemically reduced graphene porous material as light absorber for light-driven thermoelectric generator. J. Mater. Chem. 22(34), 1780017804 (2012). https://doi.org/10.1039/c2jm33530a

57. L. Liang, E. Cruz-Silva, E.C. Girao, V. Meunier, Enhanced thermoelectric figure of merit in assembled graphene nanoribbons. Phys. Rev. B 86(11), 115438 (2012). https://doi. org/10.1103/PhysRevB.86.115438

58. S. Cho, S.D. Kang, W. Kim, E.-S. Lee, S.-J. Woo et al., Thermoelectric imaging of structural disorder in epitaxial graphene. Nat. Mater. 12(10), 913-918 (2013). https://doi. org/10.1038/nmat3708

59. Y. Lu, Y. Song, F. Wang, Thermoelectric properties of graphene nanosheets-modified polyaniline hybrid nanocomposites by an in situ chemical polymerization. Mater. Chem. Phys. 138(1), 238-244 (2013). https://doi.org/10.1016/j. matchemphys.2012.11.052

60. D. Wu, K. Yan, Y. Zhou, H. Wang, L. Lin, H. Peng, Z. Liu, Plasmon-enhanced photothermoelectric conversion in chemical vapor deposited graphene $p-n$ junctions. J. Am. Chem. Soc. 135(30), 10926-10929 (2013). https://doi.org/10.1021/ ja404890n

61. T.J. Echtermeyer, P.S. Nene, M. Trushin, R.V. Gorbachev, A.L. Eiden et al., Photothermoelectric and photoelectric contributions to light detection in metal-graphene-metal photodetectors. Nano Lett. 14(7), 3733-3742 (2014). https://doi. org/10.1021/n15004762

62. Y. Xu, Z. Li, W. Duan, Thermal and thermoelectric properties of graphene. Small 10(11), 2182-2199 (2014). https://doi. org/10.1002/smll.201303701

63. P. Dollfus, N. Viet Hung, J. Saint-Martin, Thermoelectric effects in graphene nanostructures. J. Phys. Condens. Mater. 27(13), 133204 (2015). https://doi.org/10.1088/0953$8984 / 27 / 13 / 133204$

64. J.Y. Kim, J.C. Grossman, High-efficiency thermoelectrics with functionalized graphene. Nano Lett. 15(5), 2830-2835 (2015). https://doi.org/10.1021/nl504257q

65. F. Ghahari, H.-Y. Xie, T. Taniguchi, K. Watanabe, M.S. Foster, P. Kim, Enhanced thermoelectric power in graphene: violation of the mott relation by inelastic scattering. Phys. Rev. Lett. 116(13), 136802 (2016). https://doi.org/10.1103/PhysR evLett.116.136802

66. Y. Anno, Y. Imakita, K. Takei, S. Akita, T. Arie, Enhancement of graphene thermoelectric performance through defect 
engineering. 2D Mater 4(2), 025019 (2017). https://doi. org/10.1088/2053-1583/aa57fc

67. M.B. Lundeberg, Y. Gao, A. Woessner, C. Tan, P. AlonsoGonzalez et al., Thermoelectric detection and imaging of propagating graphene plasmons. Nat. Mater. 16(2), 204-207 (2017). https://doi.org/10.1038/nmat4755

68. N. Poudel, S.-J. Liang, D. Choi, B. Hou, L. Shen, H. Shi, L.K. Ang, L. Shi, S. Cronin, Cross-plane thermoelectric and thermionic transport across AU/h-BN/graphene heterostructures. Sci. Rep. 7, 14148 (2017). https://doi.org/10.1038/ s41598-017-12704-w

69. N. Yang, S. Hu, D. Ma, T. Lu, B. Li, Nanoscale graphene disk: a natural functionally graded material-how is fourier's law violated along radius direction of 2D disk. Sci. Rep. 5, 14878 (2015). https://doi.org/10.1038/srep14878

70. K.F. Mak, C. Lee, J. Hone, J. Shan, T.F. Heinz, Atomically thin $\mathrm{MoS}_{2}$ : a new direct-gap semiconductor. Phy. Rev. Lett. 105(13), 136805 (2010). https://doi.org/10.1103/PhysR evLett.105.136805

71. M. Buscema, M. Barkelid, V. Zwiller, H.S.J. van der Zant, G.A. Steele, A. Castellanos-gomez, large and tunable photothermoelectric effect in single-layer $\mathrm{MoS}_{2}$. Nano Lett. 13(2), 358-363 (2013). https://doi.org/10.1021/nl303321g

72. K. Hippalgaonkar, Y. Wang, Y. Ye, D.Y. Qiu, H. Zhu, Y. Wang, J. Moore, S.G. Louie, X. Zhang, High thermoelectric power factor in two-dimensional crystals of $\mathrm{MoS}_{2}$. Phys. Rev. B 95(11), 115407 (2017). https://doi.org/10.1103/ PhysRevB.95.115407

73. J. Hong, C. Lee, J.S. Park, J.H. Shim, Control of valley degeneracy in $\mathrm{MoS}_{2}$ by layer thickness and electric field and its effect on thermoelectric properties. Phys. Rev. B 93(3), 035445 (2016). https://doi.org/10.1103/PhysR evB.93.035445

74. J. Wu, H. Schmidt, K.K. Amara, X. Xu, G. Eda, B. Oezyilmaz, Large thermoelectricity via variable range hopping in chemical vapor deposition grown single-layer $\mathrm{MoS}_{2}$. Nano Lett. 14(5), 2730-2734 (2014). https://doi. org/10.1021/nl500666m

75. A. Molina-Sanchez, L. Wirtz, Phonons in single-layer and few-layer $\mathrm{MoS}_{2}$ and $\mathrm{WS}_{2}$. Phys. Rev. B 84(15), 155413 (2011). https://doi.org/10.1103/PhysRevB.84.155413

76. Y. Cai, J. Lan, G. Zhang, Y.-W. Zhang, Lattice vibrational modes, phonon thermal conductivity of monolayer $\mathrm{MoS}_{2}$. Phys. Rev. B 89(3), 035438 (2014). https://doi.org/10.1103/ PhysRevB.89.035438

77. C. Lee, H. Yan, L.E. Brus, T.F. Heinz, J. Hone, S. Ryu, Anomalous lattice vibrations of singleand few-layer $\mathrm{MoS}_{2}$. ACS Nano 4(5), 2695-2700 (2010). https://doi. org/10.1021/nn1003937

78. A. Taube, J. Judek, A. Lapiriska, M. Zdrojek, Temperaturedependent thermal properties of supported $\mathrm{MoS}_{2}$ monolayers. ACS Appl. Mater. Interfaces. 7(9), 5061-5065 (2015). https://doi.org/10.1021/acsami.5b00690

79. R. Yan, J.R. Simpson, S. Bertolazzi, J. Brivio, M. Watson et al., Thermal conductivity of monolayer molybdenum disulfide obtained from temperature-dependent Raman spectroscopy. ACS Nano 8(1), 986-993 (2014). https:// doi.org/10.1021/nn405826k

80. S. Sahoo, A.P.S. Gaur, M. Ahmadi, M.J.F. Guinel, R.S. Katiyar, Temperature-dependent raman studies and thermal conductivity of few-layer $\mathrm{MoS}_{2}$. J. Phys. Chem. C 117(17), 9042-9047 (2013). https://doi.org/10.1021/jp402509w

81. I. Jo, M.T. Pettes, E. Ou, W. Wu, L. Shi, Basal-plane thermal conductivity of few-layer molybdenum disulfide. Appl. Phys. Lett. 104(20), 201902 (2014). https://doi. org/10.1063/1.4876965

82. X. Liu, G. Zhang, Q.-X. Pei, Y.-W. Zhang, Phonon thermal conductivity of monolayer $\mathrm{MoS}_{2}$ sheet and nanoribbons. Appl. Phys. Lett. 103(13), 133113 (2013). https:// doi.org/10.1063/1.4823509

83. Z. Ding, J.-W. Jiang, Q.-X. Pei, Y.-W. Zhang, In-plane and cross-plane thermal conductivities of molybdenum disulfide. Nanotechnology 26(6), 065703 (2015). https:// doi.org/10.1088/0957-4484/26/6/065703

84. G. Zhang, Y.-W. Zhang, Strain effects on thermoelectric properties of two-dimensional materials. Mech. Mate. 91, 382398 (2015). https://doi.org/10.1016/j.mechmat.2015.03.009

85. W.-X. Zhou, K.-Q. Chen, First-pprinciples determination of ultralow thermal conductivity of monolayer $\mathrm{WSe}_{2}$. Sci. Rep. 5, 15070 (2015). https://doi.org/10.1038/srep15070

86. J. Ma, W. Li, X. Luo, Ballistic thermal transport in monolayer transition-metal dichalcogenides: role of atomic mass. Appl. Phys. Lett. 108(8), 082102 (2016). https://doi. org/10.1063/1.4942451

87. X. Gu, R. Yang, Phonon transport in single-layer transition metal dichalcogenides: a first-principles study. Appl. Phys. Lett. 105(13), 131903 (2014). https://doi. org/10.1063/1.4896685

88. T.-H. Liu, Y.-C. Chen, C.-W. Pao, C.-C. Chang, Anisotropic thermal conductivity of $\mathrm{MoS}_{2}$ nanoribbons: chirality, edge effects. Appl. Phys. Lett. 104(20), 201909 (2014). https://doi. org/10.1063/1.4878395

89. W. Li, J. Carrete, N. Mingo, Thermal conductivity and phonon linewidths of monolayer $\mathrm{MoS}_{2}$ from first principles. Appl. Phys. Lett. 103(25), 253103 (2013). https://doi. org/10.1063/1.4850995

90. D. Wickramaratne, F. Zahid, R.K. Lake, Electronic and thermoelectric properties of few-layer transition metal dichalcogenides. J. Chem. Phys. 140(12), 124710 (2014). https://doi. org/10.1063/1.4869142

91. M. Achimovicova, N. Daneu, A. Recnik, J. Durisin, P. Balaz, M. Fabian, J. Kovac, A. Satka, Characterization of mechanochemically synthesized lead selenide. Chem. Pap. 63(5), 562-567 (2009). https://doi.org/10.2478/s1169 6-009-0050-6

92. C. Guillen, J. Montero, J. Herrero, Characteristics of SnSe and $\mathrm{SnSe}_{2}$ thin films grown onto polycrystalline $\mathrm{SnO}_{2}$-coated glass substrates. Phys. Status Solidi A 208(3), 679-683 (2011). https://doi.org/10.1002/pssa.201026584

93. M.S. Dresselhaus, G. Chen, M.Y. Tang, R. Yang, H. Lee et al., New directions for low-dimensional thermoelectric 
materials. Adv. Mater. 19(8), 1043-1053 (2007). https://doi. org/10.1002/adma.200600527

94. J.R. Szczech, J.M. Higgins, S. Jin, Enhancement of the thermoelectric properties in nanoscale and nanostructured materials. J. Mater. Chem. 21(12), 4037-4055 (2011). https://doi. org/10.1039/c0jm02755c

95. L.D. Hicks, M.S. Dresselhaus, Thermoelectric figure of merit of a one-dimensional conductor. Phys. Rev. B 47(24), 1663116634 (1993). https://doi.org/10.1103/PhysRevB.47.16631

96. G.D. Mahan, J.O. Sofo, The best thermoelectric. Proc. Natl. Acad. Sci. U.S.A. 93(15), 7436-7439 (1996). https://doi. org/10.1073/pnas.93.15.7436

97. A. Samad, M. Noor-A-Alam, Y.-H. Shin, First principles study of a $\mathrm{SnS}_{2}$ /graphene heterostructure: a promising anode material for rechargeable Na ion batteries. J. Mater. Chem. A 4(37), 14316-14323 (2016). https://doi.org/10.1039/c6ta0 $5739 \mathrm{j}$

98. R. Fei, A. Faghaninia, R. Soklaski, J.-A. Yan, C. Lo, L. Yang, Enhanced thermoelectric efficiency via orthogonal electrical and thermal conductances in phosphorene. Nano Lett. 14(11), 6393-6399 (2014). https://doi.org/10.1021/ $\mathrm{n} 1502865 \mathrm{~s}$

99. L. Cheng, H. Liu, X. Tan, J. Zhang, J. Wei, H. Lv, J. Shi, $\mathrm{X}$. Tang, Thermoelectric properties of a monolayer bismuth. J. Phys. Chem. C 118(2), 904-910 (2014). https://doi. org/10.1021/jp411383j

100. A. Agarwal, M.N. Vashi, D. Lakshminarayana, N.M. Batra, Electrical resistivity anisotropy in layered p-SnSe single crystals. J. Mater. Sci. Mater. El. 11(1), 67-71 (2000). https://doi. org/10.1023/A:1008960305097

101. K.-M. Chung, D. Wamwangi, M. Woda, M. Wuttig, W. Bensch, Investigation of $\mathrm{SnSe}, \mathrm{SnSe}_{2}$, and $\mathrm{Sn}_{2} \mathrm{Se}_{3}$ alloys for phase change memory applications. J. Appl. Phys. 103(8), 083523 (2008). https://doi.org/10.1063/1.2894903

102. S. Chen, K. Cai, W. Zhao, The effect of Te doping on the electronic structure and thermoelectric properties of SnSe. Phys. B 407(21), 4154-4159 (2012). https://doi.org/10.1016/j. physb.2012.06.041

103. Q. Zhang, H. Wang, W. Liu, H. Wang, B. Yu et al., Enhancement of thermoelectric figure-of-merit by resonant states of aluminium doping in lead selenide. Energ. Environ. Sci. 5(1), 5246-5251 (2012). https://doi.org/10.1039/c1ee02465e

104. I.U. Arachchige, M.G. Kanatzidis, Anomalous band gap evolution from band inversion in $\mathrm{Pb}_{1-\mathrm{x}} \mathrm{Sn}_{\mathrm{x}}$ Te nanocrystals. Nano Lett. 9(4), 1583-1587 (2009). https://doi.org/10.1021/n1803 7757

105. X. Shi, J.R. Salvador, J. Yang, H. Wang, Prospective thermoelectric materials: $\left(\mathrm{AgSbTe}_{2}\right) 100_{-x}(\mathrm{SnTe})_{x}$ quaternary system $(x=80,85,90$, and 95). Sci. Adv. Mater. 3(4), 667-671 (2011). https://doi.org/10.1166/sam.2011.1198

106. R.F. Brebrick, Deviations from Stoichiometry, electrical properties in SnTe. J. Phys. Chem. Solids 24(1), 27 (1963). https://doi.org/10.1016/0022-3697(63)90038-6

107. Y. Chen, M.D. Nielsen, Y.-B. Gao, T.-J. Zhu, X. Zhao, J.P. Heremans, SnTe-AgSbTe 2 thermoelectric alloys. Adv.
Energy Mater. 2(1), 58-62 (2012). https://doi.org/10.1002/ aenm.201100460

108. M.-K. Han, J. Androulakis, S.-J. Kim, M.G. Kanatzidis, Lead-free thermoelectrics: high figure of merit in $p$-type $\mathrm{AgSn}_{\mathrm{m}} \mathrm{SbTe}_{\mathrm{m}+2}$. Adv. Energy Mater. 2(1), 157-161 (2012). https://doi.org/10.1002/aenm.201100613

109. Z.G. Chen, X.L. Shi, L.D. Zhao, J. Zou, High-performance SnSe thermoelectric materials: progress and future challenge. Prog. Mater Sci. 97, 283-346 (2018). https://doi. org/10.1016/j.pmatsci.2018.04.005

110. L.-D. Zhao, C. Chang, G. Tan, M.G. Kanatzidis, SnSe: a remarkable new thermoelectric material. Energy Environ. Sci. 9(10), 3044-3060 (2016). https://doi.org/10.1039/c6ee0 $1755 \mathrm{j}$

111. J.A. Kafalas, R.F. Brebrick, A.J. Strauss, Evidence that snte is semiconductor energy band model thermoelectric preparation at high pressure. Appl. Phys. Lett. 4(5), 93 (1964). https://doi. org/10.1063/1.1753977

112. S.C. Dhanabalan, J.S. Ponraj, Z. Guo, S. Li, Q. Bao, H. Zhang, Emerging trends in phosphorene fabrication towards next generation devices. Adv. Sci. 4(6), 1600305 (2017). https://doi.org/10.1002/advs.201600305

113. H.O. Churchill, P. Jarillo-Herrero, Two-dimensional crystals: phosphorus joins the family. Nat. Nanotechnol. 9(5), 330-331 (2014). https://doi.org/10.1038/nnano.2014.85

114. M. Zhang, Q. Wu, F. Zhang, L. Chen, X. Jin, Y. Hu, Z. Zheng, H. Zhang, 2D black phosphorus saturable absorbers for ultrafast photonics. Adv. Opt. Mater. 7(1), 1800224 (2019). https ://doi.org/10.1002/adom.201800224

115. Z. Li, H. Xu, J. Shao, C. Jiang, F. Zhang, J. Lin, H. Zhang, J. Li, P. Huang, Polydopamine-functionalized black phosphorus quantum dots for cancer theranostics. Appl. Mater. Today 15, 297-304 (2019). https://doi.org/10.1016/j.apmt.2019.02.002

116. R. Cao, H.D. Wang, Z.N. Guo, D.K. Sang, L.Y. Zhang et al., Black phosphorous/indium selenide photoconductive detector for visible and near-infrared light with high sensitivity. Adv. Opt. Mater. 7(12), 1900020 (2019). https://doi.org/10.1002/ adom. 201900020

117. J. Qiao, X. Kong, Z.-X. Hu, F. Yang, W. Ji, High-mobility transport anisotropy and linear dichroism in few-layer black phosphorus. Nat. Commun. 5, 4475 (2014). https://doi. org/10.1038/ncomms5475

118. R. Fei, L. Yang, Strain-engineering the anisotropic electrical conductance of few-layer black phosphorus. Nano Lett. 14(5), 2884-2889 (2014). https://doi.org/10.1021/nl500935z

119. H. Wang, X. Wang, F. Xia, L. Wang, H. Jiang, Q. Xia, M.L. Chin, M. Dubey, S.-J. Han, Black phosphorus radiofrequency transistors. Nano Lett. 14(11), 6424-6429 (2014). https://doi.org/10.1021/n15029717

120. W. Huang, Z. Xie, T. Fan, J. Li, Y. Wang et al., Black-phosphorus-analogue tin monosulfide: an emerging optoelectronic two-dimensional material for high-performance photodetection with improved stability under ambient/harsh conditions. J. Mater. Chem. C 6(36), 9582-9593 (2018). https://doi. org/10.1039/c8tc03284j 
121. L. Viti, J. Hu, D. Coquillat, A. Politano, W. Knap, M.S. Vitiello, Efficient terahertz detection in black-phosphorus nano-transistors with selective and controllable plasma-wave, bolometric and thermoelectric response. Sci. Rep. 6, 20474 (2016). https://doi.org/10.1038/srep20474

122. M. Qiu, D. Wang, W. Liang, L. Liu, Y. Zhang et al., Novel concept of the smart NIR-light-controlled drug release of black phosphorus nanostructure for cancer therapy. Proc. Natl. Acad. Sci. U.S.A. 115(3), 501-506 (2018). https://doi. org/10.1073/pnas.1714421115

123. C. Xing, S. Chen, M. Qiu, X. Liang, Q. Liu et al., Conceptually novel black phosphorus/cellulose hydrogels as promising photothermal agents for effective cancer therapy. Adv. Healthc. Mater. 7(7), 1701510 (2018). https://doi. org/10.1002/adhm.201701510

124. S. Luo, J. Zhao, J. Zou, Z. He, C. Xu, F. Liu, Y. Huang, L. Dong, L. Wang, H. Zhang, Self-standing polypyrrole/black phosphorus laminated film: promising electrode for flexible supercapacitor with enhanced capacitance and cycling stability. ACS Appl. Mater. Interfaces. 10(4), 3538-3548 (2018). https://doi.org/10.1021/acsami.7b15458

125. H.-D. Wang, D.K. Sang, Z.-N. Guo, R. Cao, J.-L. Zhao et al., Black phosphorus-based field effect transistor devices for Ag ions detection. Chin. Phys. B 27(8), 087308 (2018). https://doi.org/10.1088/1674-1056/27/8/087308

126. T. Hong, B. Chamlagain, W. Lin, H.-J. Chuang, M. Pan, Z. Zhou, Y.-Q. Xu, Polarized photocurrent response in black phosphorus field-effect transistors. Nanoscale 6(15), 8978-8983 (2014). https://doi.org/10.1039/c4nr02164a

127. J. Sun, H.-W. Lee, M. Pasta, H. Yuan, G. Zheng, Y. Sun, Y. Li, Y. Cui, A phosphorene-graphene hybrid material as a high-capacity anode for sodium-ion batteries. Nat. Nanotechnol. 10(11), 980-985 (2015). https://doi.org/10.1038/ nnano.2015.194

128. J. Sun, G. Zheng, H.-W. Lee, N. Liu, H. Wang, H. Yao, W. Yang, Y. Cui, Formation of stable phosphorus-carbon bond for enhanced performance in black phosphorus nanoparticle-graphite composite battery anodes. Nano Lett. 14(8), 4573-4580 (2014). https://doi.org/10.1021/n1501617j

129. C.J. An, Y.H. Kang, C. Lee, S.Y. Cho, Preparation of highly stable black phosphorus by gold decoration for high-performance thermoelectric generators. Adv. Funct. Mater. 28(28), 1800532 (2018). https://doi.org/10.1002/ adfm.201800532

130. E. Flores, J.R. Ares, A. Castellanos-Gomez, M. Barawi, I.J. Ferrer, C. Sanchez, Thermoelectric power of bulk blackphosphorus. Appl. Phys. Lett. 106(2), 022102 (2015). https ://doi.org/10.1063/1.4905636

131. S.J. Choi, B.-K. Kim, T.-H. Lee, Y.H. Kim, Z. Li et al., Electrical and thermoelectric transport by variable range hopping in thin black phosphorus devices. Nano Lett. 16(7), 39693975 (2016). https://doi.org/10.1021/acs.nanolett.5b04957

132. J. Zhang, H.J. Liu, L. Cheng, J. Wei, J.H. Liang et al., High thermoelectric performance can be achieved in black phosphorus. J. Mater. Chem. C 4(5), 991-998 (2016). https://doi. org/10.1039/c5tc03238e
133. G. Qin, Q.-B. Yan, Z. Qin, S.-Y. Yue, H.-J. Cui, Q.-R. Zheng, $\mathrm{G}$. $\mathrm{Su}$, Hinge-like structure induced unusual properties of black phosphorus and new strategies to improve the thermoelectric performance. Sci. Rep. 4, 6946 (2014). https://doi. org/10.1038/srep06946

134. Y. Saito, T. Iizuka, T. Koretsune, R. Arita, S. Shimizu, Y. Iwasa, Gate-tuned thermoelectric power in black phosphorus. Nano Lett. 16(8), 4819-4824 (2016). https://doi.org/10.1021/ acs.nanolett.6b00999

135. B. Smith, B. Vermeersch, J. Carrete, E. Ou, J. Kim, N. Mingo, D. Akinwande, L. Shi, Temperature and thickness dependences of the anisotropic in-plane thermal conductivity of black phosphorus. Adv. Mater. 29(5), 1603756 (2017). https ://doi.org/10.1002/adma.201603756

136. B. Sun, X. Gu, Q. Zeng, X. Huang, Y. Yan, Z. Liu, R. Yang, Y.K. Koh, Temperature dependence of anisotropic thermalconductivity tensor of bulk black phosphorus. Adv. Mater. 29(3), 1603297 (2017). https://doi.org/10.1002/adma.20160 3297

137. S. Lee, F. Yang, J. Suh, S. Yang, Y. Lee et al., Anisotropic in-plane thermal conductivity of black phosphorus nanoribbons at temperatures higher than $100 \mathrm{~K}$. Nat. Commun. 6, 8573 (2015). https://doi.org/10.1038/ncomms9573

138. Z. Luo, J. Maassen, Y. Deng, Y. Du, R.P. Garrelts, M.S. Lundstrom, P.D. Ye, X. Xu, Anisotropic in-plane thermal conductivity observed in few-layer black phosphorus. Nat. Commun. 6, 8572 (2015). https://doi.org/10.1038/ncomm s9572

139. A. Jain, A.J.H. McGaughey, Strongly anisotropic in-plane thermal transport in single-layer black phosphorene. Sci. Rep. 5, 8501 (2015). https://doi.org/10.1038/srep08501

140. H. Jang, J.D. Wood, C.R. Ryder, M.C. Hersam, D.G. Cahill, Anisotropic thermal conductivity of exfoliated black phosphorus. Adv. Mater. 27(48), 8017-8022 (2015). https://doi. org/10.1002/adma.201503466

141. M. Khazaei, M. Arai, T. Sasaki, M. Estili, Y. Sakka, Twodimensional molybdenum carbides: potential thermoelectric materials of the MXene family. Phys. Chem. Chem. Phys. 16(17), 7841-7849 (2014). https://doi.org/10.1039/ c4cp00467a

142. M. Naguib, J. Halim, J. Lu, K.M. Cook, L. Hultman, Y. Gogotsi, M.W. Barsoum, New two-dimensional niobium and vanadium carbides as promising materials for Li-ion batteries. J. Am. Chem. Soc. 135(43), 15966-15969 (2013). https://doi.org/10.1021/ja405735d

143. M.R. Lukatskaya, O. Mashtalir, C.E. Ren, Y. Dall'Agnese, P. Rozier et al., Cation intercalation and high volumetric capacitance of two-dimensional titanium carbide. Science 341(6153), 1502-1505 (2013). https://doi.org/10.1126/ science. 1241488

144. M. Naguib, M. Kurtoglu, V. Presser, J. Lu, J. Niu et al., Two-dimensional nanocrystals produced by exfoliation of $\mathrm{Ti}_{3} \mathrm{AlC}_{2}$. Adv. Mater. 23(37), 4248-4253 (2011). https:// doi.org/10.1002/adma.201102306 
145. O. Mashtalir, M. Naguib, V.N. Mochalin, Y. Dall'Agnese, M. Heon, M.W. Barsoum, Y. Gogotsi, Intercalation and delamination of layered carbides and carbonitrides. Nat. Commun. 4, 1716 (2013). https://doi.org/10.1038/ncomm s2664

146. S. Kumar, U. Schwingenschlogl, Thermoelectric performance of functionalized $\mathrm{Sc}_{2} \mathrm{C}$ MXenes. Phys. Rev. B 94(3), 035405 (2016). https://doi.org/10.1103/PhysRevB.94.035405

147. H. Kim, B. Anasori, Y. Gogotsi, H.N. Alshareef, Thermoelectric properties of two-dimensional molybdenum-based MXenes. Chem. Mater. 29(15), 6472-6479 (2017). https:// doi.org/10.1021/acs.chemmater.7b02056

148. S. Sarikurt, D. Cakir, M. Keceli, C. Sevik, The influence of surface functionalization on thermal transport and thermoelectric properties of MXene monolayers. Nanoscale 10(18), 8859-8868 (2018). https://doi.org/10.1039/c7nr09144c

149. S. Kumar, U. Schwingenschlogl, Thermoelectric response of bulk and mono layer $\mathrm{MoSe}_{2}$ and $\mathrm{WSe}_{2}$. Chem. Mater. 27(4), 1278-1284 (2015). https://doi.org/10.1021/cm504244b

150. S. Chandra, A. Banik, K. Biswas, N-type ultrathin few-layer nanosheets of bi-doped SnSe: synthesis and thermoelectric properties. ACS Energy Lett. 3(5), 1153-1158 (2018). https ://doi.org/10.1021/acsenergylett.8b00399

151. G. Han, S.R. Popuri, H.F. Greer, J.-W.G. Bos, W. Zhou et al., Facile surfactant-free synthesis of p-type SnSe nanoplates with exceptional thermoelectric power factors. Angew. Chem. Int. Ed. 55(22), 6433-6437 (2016). https:// doi.org/10.1002/anie.201601420

152. N.A. Rongione, M. Li, H. Wu, H.D. Nguyen, J.S. Kang, B. Ouyang, H. Xia, Y. Hu, High-performance solutionprocessable flexible SnSe nanosheet films for lower grade waste heat recovery. Adv. Electron. Mater. 5(3), 1800774 (2019). https://doi.org/10.1002/aelm.201800774

153. H. Ju, M. Kim, D. Park, J. Kim, A strategy for low thermal conductivity and enhanced thermoelectric performance in SnSe: porous $\mathrm{SnSe}_{1-\mathrm{x}} \mathrm{S}_{\mathrm{x}}$ nanosheets. Chem. Mater. 29(7), 3228-3236 (2017). https://doi.org/10.1021/acs.chemm ater.7b00423

154. M. Hong, Z.-G. Chen, L. Yang, T.C. Chasapis, S.D. Kang et al., Enhancing the thermoelectric performance of $\mathrm{SnSe}_{1-\mathrm{x}} \mathrm{Te}_{\mathrm{x}}$ nanoplates through band engineering. J. Mater. Chem. A 5(21), 10713-10721 (2017). https://doi. org/10.1039/c7ta02677c

155. H. Ju, K. Kim, D. Park, J. Kim, Fabrication of porous SnSeS nanosheets with controlled porosity and their enhanced thermoelectric performance. Chem. Eng. J. 335, 560-566 (2018). https://doi.org/10.1016/j.cej.2017.11.003

156. P. Xu, T. Fu, J. Xin, Y. Liu, P. Ying, X. Zhao, H. Pan, T. Zhu, Anisotropic thermoelectric properties of layered compound $\mathrm{SnSe}_{2}$. Sci. Bull. 62(24), 1663-1668 (2017). https:// doi.org/10.1016/j.scib.2017.11.015

157. Y. Wu, W. Li, A. Faghaninia, Z. Chen, J. Li et al., Promising thermoelectric performance in van der Waals layered $\mathrm{SnSe}_{2}$. Mater. Today Phys. 3, 127-136 (2017). https://doi. org/10.1016/j.mtphys.2017.10.001
158. Y. Shu, X. Su, H. Xie, G. Zheng, W. Liu, Y. Yan, T. Luo, X. Yang, D. Yang, C. Uher, X. Tang, Modification of bulk heterojunction and $\mathrm{Cl}$ doping for high-performance thermoelectric $\mathrm{SnSe}_{2} / \mathrm{SnSe}$ nanocomposites. ACS Appl. Mater. Interfaces 10(18), 15793-15802 (2018). https://doi. org/10.1021/acsami.8b00524

159. S. Saha, A. Banik, K. Biswas, Few-layer nanosheets of n-type $\mathrm{SnSe}_{2}$. Chem. Eur. J. 22(44), 15634-15638 (2016). https://doi.org/10.1002/chem.201604161

160. X. Yan, X. Cui, B. Li, L.-S. Li, Large, solution-processable graphene quantum dots as light absorbers for photovoltaics. Nano Lett. 10(5), 1869-1873 (2010). https://doi. org/10.1021/nl101060h

161. J.S. Son, M.K. Choi, M.-K. Han, K. Park, J.-Y. Kim et al., $\mathrm{N}$-type nanostructured thermoelectric materials prepared from chemically synthesized ultrathin $\mathrm{Bi}_{2} \mathrm{Te}_{3}$ nanoplates. Nano Lett. 12(2), 640-647 (2012). https://doi.org/10.1021/ n1203389x

162. Y. Min, G. Park, B. Kim, A. Giri, J. Zeng, J.W. Roh, S.I. Kim, K.H. Lee, U. Jeong, Synthesis of multishell nanoplates by consecutive epitaxial growth of $\mathrm{Bi}_{2} \mathrm{Se}_{3}$ and $\mathrm{Bi}_{2} \mathrm{Te}_{3}$ nanoplates and enhanced thermoelectric properties. ACS Nano 9(7), 6843-6853 (2015). https://doi.org/10.1021/ $\mathrm{nn} 507250 \mathrm{r}$

163. M. Hong, T.C. Chasapis, Z.-G. Chen, L. Yang, M.G. Kanatzidis, G.J. Snyder, J. Zou, N-type $\mathrm{Bi}_{2} \mathrm{Te}_{3-\mathrm{x}} \mathrm{Se}_{\mathrm{x}}$ nanoplates with enhanced thermoelectric efficiency driven by wide-fequency phonon scatterings and synergistic carrier scatterings. ACS Nano 10(4), 4719-4727 (2016). https:// doi.org/10.1021/acsnano.6b01156

164. Z. Lu, M. Layani, X. Zhao, L.P. Tan, T. Sun, S. Fan, Q. Yan, S. Magdassi, H.H. Hng, Fabrication of flexible thermoelectric thin film devices by inkjet printing. Small 10(17), 3551-3554 (2014). https://doi.org/10.1002/smll.201303126

165. Z. Aabdin, N. Peranio, M. Winkler, D. Bessas, J. König, R.P. Hermann, H. Böttner, O. Eibl, $\mathrm{Sb}_{2} \mathrm{Te}_{3}$ and $\mathrm{Bi}_{2} \mathrm{Te}_{3}$ thin films grown by room-temperature MBE. J. Electron. Mater. 41(6), 1493-1497 (2011). https://doi.org/10.1007/s1166 4-011-1870-Z

166. H. You, S.-H. Bae, J. Kim, J.-S. Kim, C. Park, Deposition of nanocrystalline $\mathrm{Bi}_{2} \mathrm{Te}_{3}$ films using a modified MOCVD system. J. Electron. Mater. 40(5), 635-640 (2011). https:// doi.org/10.1007/s11664-010-1490-Z

167. X. Tang, H. Chen, J.S. Ponraj, S.C. Dhanabalan, Q. Xiao, D. Fan, H. Zhang, Fluorination-enhanced ambient stability and electronic tolerance of black phosphorus quantum dots. Adv. Sci. 5(9), 1800420 (2018). https://doi.org/10.1002/ advs. 201800420

168. X. Zhongjian, W. Dou, F. Taojian, X. Chenyang, L. Zhongjun et al., Black phosphorus analogue tin sulfide nanosheets: synthesis and application as near-infrared photothermal agents and drug delivery platforms for cancer therapy. J. Mater. Chem. B 6(29), 4747-4755 (2018). https ://doi.org/10.1039/c8tb00729b

169. J. Zhou, Z. Li, M. Ying, M. Liu, X. Wang, X. Wang, L. Cao, H. Zhang, G. Xu, Black phosphorus nanosheets for 
rapid microRNA detection. Nanoscale 10(11), 5060-5064 (2018). https://doi.org/10.1039/c7nr08900g

170. L. Wu, Z. Xie, L. Lu, J. Zhao, Y. Wang et al., Few-layer tin sulfide: a promising black-phosphorus-analogue $2 \mathrm{D}$ material with exceptionally large nonlinear optical response, hgh stability, and applications in all-optical switching and wavelength conversion. Adv. Opt. Mater. 6(2), 1700985 (2018). https://doi.org/10.1002/adom.201700985

171. Y. Zhou, M. Zhang, Z. Guo, L. Miao, S.-T. Han, Z. Wang, X. Zhang, H. Zhang, Z. Peng, Recent advances in black phosphorus-based photonics, electronics, sensors and energy devices. Mater. Horizons 4(6), 997-1019 (2017). https://doi.org/10.1039/c7mh00543a

172. Y. Xu, J. Yuan, K. Zhang, Y. Hou, Q. Sun et al., Fieldinduced n-doping of black phosphorus for CMOS compatible 2D logic electronics with high electron mobility. Adv. Funct. Mater. 27(38), 1702211 (2017). https://doi. org/10.1002/adfm.201702211

173. X. Ren, J. Zhou, X. Qi, Y. Liu, Z. Huang et al., Few-layer black phosphorus nanosheets as electrocatalysts for highly efficient oxygen evolution reaction. Adv. Energy Mater. 7(19), 1700396 (2017). https://doi.org/10.1002/aenm.20170 0396

174. M. Qiu, Z.T. Sun, D.K. Sang, X.G. Han, H. Zhang, C.M. Niu, Current progress in black phosphorus materials and their applications in electrochemical energy storage. Nanoscale 9(36), 13384-13403 (2017). https://doi.org/10.1039/c7nr0 $3318 \mathrm{~d}$

175. G. Eda, H. Yamaguchi, D. Voiry, T. Fujita, M. Chen, M. Chhowalla, Photoluminescence from chemically exfoliated $\mathrm{MoS}_{2}$. Nano Lett. 11(12), 5111-5116 (2011). https://doi. org/10.1021/nl201874w

176. J. Pu, Y. Yomogida, K.-K. Liu, L.-J. Li, Y. Iwasa, T. Takenobu, Highly flexible $\mathrm{MoS}_{2}$ thin-film transistors with ion gel dielectrics. Nano Lett. 12(8), 4013-4017 (2012). https:// doi.org/10.1021/nl301335q

177. B. Radisavljevic, A. Radenovic, J. Brivio, V. Giacometti, A. Kis, Single-layer $\mathrm{MoS}_{2}$ transistors. Nat. Nanotechnol. 6(3), 147-150 (2011). https://doi.org/10.1038/nnano.2010.279

178. J. Feng, W. Zhu, Y. Deng, Q. Song, Q. Zhang, Enhanced antioxidation and thermoelectric properties of the flexible screen-printed $\mathrm{Bi}_{2} \mathrm{Te}_{3}$ films through interface modification. ACS Appl. Energy Mater. 2(4), 2828-2836 (2019). https:// doi.org/10.1021/acsaem.9b00178

179. M.R. Scimeca, F. Yang, E. Zaia, N. Chen, P. Zhao et al., Rapid stoichiometry control in $\mathrm{Cu}_{2} \mathrm{Se}$ thin films for roomtemperature power factor improvement. ACS Appl. Energy Mater. 2(2), 1517-1525 (2019). https://doi.org/10.1021/acsae m. 8 b02118

180. A. Bano, N.K. Gaur, Interfacial coupling effect on electron transport in $\mathrm{MoS}_{2} / \mathrm{SrTiO}_{3}$ heterostructure: an ab initio study. Sci. Rep. 8, 714 (2018). https://doi.org/10.1038/s41598-01718984-6

181. H. Huang, Y. Cui, Q. Li, C. Dun, W. Zhou, W. Huang, L. Chen, C.A. Hewitt, D.L. Carroll, Metallic 1T phase $\mathrm{MoS}_{2}$ nanosheets for high-performance thermoelectric energy harvesting. Nano Energy 26, 172-179 (2016). https://doi. org/10.1016/j.nanoen.2016.05.022

182. Y. Guo, C. Dun, J. Xu, P. Li, W. Huang et al., Wearable thermoelectric devices based on Au-decorated two-dimensional $\mathrm{MoS}_{2}$. ACS Appl. Mater. Interfaces 10(39), 33316-33321 (2018). https://doi.org/10.1021/acsami.8b10720

183. Y. Zhou, J. Wan, Q. Li, L. Chen, J. Zhou et al., Chemical welding on semimetallic $\mathrm{TiS}_{2}$ nanosheets for high-performance flexible n-type thermoelectric films. ACS Appl. Mater. Interfaces 9(49), 42430-42437 (2017). https://doi. org/10.1021/acsami.7b15026

184. R. Tian, C. Wan, Y. Wang, Q. Wei, T. Ishida et al., A solutionprocessed $\mathrm{TiS}_{2}$ /organic hybrid superlattice film towards flexible thermoelectric devices. J. Mater. Chem. A 5(2), 564-570 (2017). https://doi.org/10.1039/c6ta08838d

185. C. Wan, Y. Kodama, M. Kondo, R. Sasai, X. Qian et al., Dielectric mismatch mediates carrier mobility in organicintercalated layered $\mathrm{TiS}_{2}$. Nano Lett. 15(10), 6302-6308 (2015). https://doi.org/10.1021/acs.nanolett.5b01013

186. C. Wan, X. Gu, F. Dang, T. Itoh, Y. Wang et al., Flexible n-type thermoelectric materials by organic intercalation of layered transition metal dichalcogenide $\mathrm{TiS}_{2}$. Nat. Mater. 14(6), 622-627 (2015). https://doi.org/10.1038/nmat4251

187. Y. Lin, C. Norman, D. Srivastava, F. Azough, L. Wang et al., Thermoelectric power generation from lanthanum strontium titanium oxide at room temperature through the addition of graphene. ACS Appl. Mater. Interfaces 7(29), 15898-15908 (2015). https://doi.org/10.1021/acsami.5b035 22

188. D. Chen, Y. Zhao, Y. Chen, B. Wang, H. Chen, J. Zhou, Z. Liang, One-step chemical synthesis of $\mathrm{ZnO} /$ Graphene Oxide molecular hybrids for high-temperature thermoelectric applications. ACS Appl. Mater. Interfaces 7(5), 3224-3230 (2015). https://doi.org/10.1021/am507882f

189. S. Li, T. Fan, X. Liu, F. Liu, H. Meng, Y. Liu, F. Pan, Graphene quantum dots embedded in $\mathrm{Bi}_{2} \mathrm{Te}_{3}$ nanosheets to enhance thermoelectric performance. ACS Appl. Mater. Interfaces 9(4), 3677-3685 (2017). https://doi.org/10.1021/ acsami.6b14274

190. J. Dong, W. Liu, H. Li, X. Su, X. Tang, C. Uher, In situ synthesis and thermoelectric properties of $\mathrm{PbTe}$-graphene nanocomposites by utilizing a facile and novel wet chemical method. J. Mater. Chem. A 1(40), 12503-12511 (2013). https ://doi.org/10.1039/c3ta12494k

191. D. Zhao, X. Wang, D. Wu, Enhanced thermoelectric properties of graphene/ $\mathrm{Cu}_{2} \mathrm{SnSe}_{3}$ composites. Crystals 7(3), 71 (2017). https://doi.org/10.3390/cryst7030071

192. H. Chen, C. Yang, H. Liu, G. Zhang, D. Wan, F. Huang, Thermoelectric properties of $\mathrm{CuInTe}_{2} /$ graphene composites. CrystEngComm 15(34), 6648-6651 (2013). https://doi. org/10.1039/c3ce40334c

193. B. Feng, J. Xie, G. Cao, T. Zhu, X. Zhao, Enhanced thermoelectric properties of p-type $\mathrm{CoSb}_{3}$ /graphene nanocomposite. J. Mater. Chem. A 1(42), 13111-13119 (2013). https://doi. org/10.1039/c3ta13202a 
194. S. Yang, J. Si, Q. Su, H. Wu, Enhanced thermoelectric performance of $\mathrm{SnSe}$ doped with layered $\mathrm{MoS}_{2} /$ graphene. Mater. Lett. 193, 146-149 (2017). https://doi.org/10.1016/j.matle t.2017.01.079

195. D. Suh, S. Lee, H. Mun, S.-H. Park, K.H. Lee, S.W. Kim, J.-Y. Choi, S. Baik, Enhanced thermoelectric performance of $\mathrm{Bi}_{0.5} \mathrm{Sb}_{1.5} \mathrm{Te}_{3}$-expanded graphene composites by simultaneous modulation of electronic and thermal carrier transport. Nano Energy 13, 67-76 (2015). https://doi.org/10.1016/j.nanoe n.2015.02.001

196. S. Li, X. Liu, Y. Liu, F. Liu, J. Luo, F. Pan, Optimized heterointerfaces by tuning $2 \mathrm{D} \mathrm{SnS}$ thickness in $\mathrm{Bi}_{2} \mathrm{Te}_{2.7} \mathrm{Se}_{0.3} / \mathrm{SnS}_{2}$ nanocomposites to enhance thermoelectric performance. Nano Energy 39, 297-305 (2017). https://doi.org/10.1016/j. nanoen.2017.07.011

197. X.-Q. Huang, Y.-X. Chen, M. Yin, D. Feng, J. He, Origin of the enhancement in transport properties on polycrystalline SnSe with compositing two-dimensional material $\mathrm{MoSe}_{2}$. Nanotechnology 28(10), 105708 (2017). https://doi. org/10.1088/1361-6528/aa55e3

198. Z.U. Khan, J. Edberg, M.M. Hamedi, R. Gabrielsson, H. Granberg et al., Thermoelectric polymers and their elastic aerogels. Adv. Mater. 28(22), 4556-4562 (2016). https://doi. org/10.1002/adma.201505364

199. M. He, F. Qiu, Z. Lin, Towards high-performance polymerbased thermoelectric materials. Energy Environ. Sci. 6(5), 1352-1361 (2013). https://doi.org/10.1039/c3ee24193a

200. X. Hu, K. Zhang, J. Zhang, S. Wang, Y. Qiu, Thermoelectric properties of conducting polymer nanowire-tellurium nanowire composites. ACS Appl. Energy Mater. 1(9), 4883-4890 (2018). https://doi.org/10.1021/acsaem.8b00909

201. A.S. Kshirsagar, C. Hiragond, A. Dey, P.V. More, P.K. Khanna, Band engineered I/III/V-VI binary metal selenide/ MWCNT/PANI nanocomposites for potential room temperature thermoelectric applications. ACS Appl. Energy Mater. 2(4), 2680-2691 (2019). https://doi.org/10.1021/acsae m.9b00013

202. X. Wang, F. Meng, Q. Jiang, W. Zhou, F. Jiang et al., Simple layer-by-layer assembly method for simultaneously enhanced electrical conductivity and thermopower of PEDOT:PSS/Ce$\mathrm{MoS}_{2}$ heterostructure films. ACS Appl. Energy Mater. 1(7), 3123-3133 (2018). https://doi.org/10.1021/acsaem.8b00315

203. Y. Chen, Y. Zhao, Z. Liang, Solution processed organic thermoelectrics: towards flexible thermoelectric modules. Energy Environ. Sci. 8(2), 401-422 (2015). https://doi.org/10.1039/ c4ee3297g

204. B. Russ, A. Glaudell, J.J. Urban, M.L. Chabinyc, R.A. Segalman, Organic thermoelectric materials for energy harvesting, temperature control. Nat. Rev. Mater. 1(10), 16050 (2016). https://doi.org/10.1038/natrevmats.2016.50

205. H. Ju, J. Kim, Chemically exfoliated SnSe nanosheets and their $\mathrm{SnSe} / \mathrm{Poly}(3,4$-ethylenedioxythiophene):poly(styrenes ulfonate) composite films for polymer based thermoelectric applications. ACS Nano 10(6), 5730-5739 (2016). https:// doi.org/10.1021/acsnano.5b07355
206. N.E. Coates, S.K. Yee, B. McCulloch, K.C. See, A. Majumdar, R.A. Segalman, J.J. Urban, Effect of interfacial properties on polymer-nanocrystal thermoelectric transport. Adv. Mater. 25(11), 1629-1633 (2013). https://doi.org/10.1002/ adma.201203915

207. L. Wang, Q. Yao, J. Xiao, K. Zeng, S. Qu, W. Shi, Q. Wang, L. Chen, Engineered molecular chain ordering in singlewalled carbon nanotubes/polyaniline composite films for high-performance organic thermoelectric materials. Chem. Asian J. 11(12), 1804-1810 (2016). https://doi.org/10.1002/ asia.201600212

208. J. Choi, J.Y. Lee, S.S. Lee, C.R. Park, H.J.A.E.M. Kim, High-performance thermoelectric paper based on double carrier-filtering processes at nanowire heterojunctions. Adv. Energy Mater. 6(9), 1502181 (2016). https://doi.org/10.1002/ aenm.201502181

209. E.W. Zaia, A. Sahu, P. Zhou, M.P. Gordon, J.D. Forster et al., Carrier scattering at alloy nanointerfaces enhances power factor in PEDOT:PSS hybrid thermoelectrics. Nano Lett. 16(5), 3352-3359 (2016). https://doi.org/10.1021/acs.nanol ett.6b01009

210. H. Ju, D. Park, J. Kim, Fabrication of porous SnS nanosheets and their combination with conductive polymer for hybrid thermoelectric application. Chem. Eng. J. 356, 950-954 (2019). https://doi.org/10.1016/j.cej.2018.09.106

211. H. Ju, J. Kim, Fabrication of conductive polymer/inorganic nanoparticles composite films: PEDOT:PSS with exfoliated tin selenide nanosheets for polymer-based thermoelectric devices. Chem. Eng. J. 297, 66-73 (2016). https://doi. org/10.1016/j.cej.2016.03.137

212. H. Ju, D. Park, J. Kim, Fabrication of polyaniline-coated SnSeS Nanosheet/Polyvinylidene difluoride composites by a solution-based process and optimization for flexible thermoelectrics. ACS Appl. Mater. Interfaces. 10(14), 1192011925 (2018). https://doi.org/10.1021/acsami.7b19667

213. H. Ju, D. Park, J. Kim, Solution-processable flexible thermoelectric composite films based on conductive polymer/ $\mathrm{SnSe}_{0.8} \mathrm{~S}_{0.2}$ nanosheets/carbon nanotubes for wearable electronic applications. J. Mater. Chem. 6(14), 5627-5634 (2018). https://doi.org/10.1039/c7ta11285h

214. X. Cheng, L. Wang, X. Wang, G. Chen, Flexible films of poly(3,4-ethylenedioxythiophene):poly(styrenesulfonate)/ SnS nanobelt thermoelectric composites. Compos. Sci. Technol. 155, 247-251 (2018). https://doi.org/10.1016/j. compscitech.2017.12.028

215. B. Zhang, J. Sun, H.E. Katz, F. Fang, R.L. Opila, Promising thermoelectric properties of commercial PEDOT:PSS materials and their $\mathrm{Bi}_{2} \mathrm{Te}_{3}$ powder composites. ACS Appl. Mater. Interfaces 2(11), 3170-3178 (2010). https://doi. org/10.1021/am100654p

216. Y. Du, K.F. Cai, S. Chen, P. Cizek, T. Lin, Facile preparation and thermoelectric Properties of $\mathrm{Bi}_{2} \mathrm{Te}_{3}$ based alloy nanosheet/PEDOT:PSS composite films. ACS Appl. Mater. Interfaces 6(8), 5735-5743 (2014). https://doi.org/10.1021/ am5002772 
217. F. Jiang, J. Xiong, W. Zhou, C. Liu, L. Wang, F. Zhao, H. Liu, J. Xu, Use of organic solvent-assisted exfoliated $\mathrm{MoS}_{2}$ for optimizing the thermoelectric performance of flexible PEDOT:PSS thin films. J. Mater. Chem. A 4(14), 5265-5273 (2016). https://doi.org/10.1039/c6ta00305b

218. E.J. Bae, Y.H. Kang, K.-S. Jang, C. Lee, S.Y. Cho, Solution synthesis of telluride-based nano-barbell structures coated with PEDOT:PSS for spray-printed thermoelectric generators. Nanoscale 8(21), 10885-10890 (2016). https:// doi.org/10.1039/c5nr07032e

219. X. Li, C. Liu, T. Wang, W. Wang, X. Wang, Q. Jiang, F. Jiang, J. Xu, Preparation of 2D MoSe ${ }_{2} /$ PEDOT:PSS composite and its thermoelectric properties. Mater. Res. Express 4(11), 116410 (2017). https://doi.org/10.1088/2053-1591/ aa99f9

220. Y. Wang, J. Yang, L. Wang, K. Du, Q. Yin, Q. Yin, Polypyrrole/graphene/polyaniline ternary nanocomposite with high thermoelectric power factor. ACS Appl. Mater. Interfaces 9(23), 20124-20131 (2017). https://doi.org/10.1021/acsam i. $7 \mathrm{~b} 05357$

221. L. Wang, Q. Yao, H. Bi, F. Huang, Q. Wang, L. Chen, PANI/ graphene nanocomposite films with high thermoelectric properties by enhanced molecular ordering. J. Mater. Chem. A 3(13), 7086-7092 (2015). https://doi.org/10.1039/c4ta06422d

222. Z. Zhang, G. Chen, H. Wang, W. Zhai, Enhanced thermoelectric property by the construction of a nanocomposite 3D interconnected architecture consisting of graphene nanolayers sandwiched by polypyrrole nanowires. J. Mater. Chem. C 3(8), 1649-1654 (2015). https://doi.org/10.1039/c4tc0 $2471 \mathrm{k}$

223. K. Xu, G. Chen, D. Qiu, Convenient construction of poly(3,4ethylenedioxythiophene)-graphene pie-like structure with enhanced thermoelectric performance. J. Mater. Chem. A 1(40), 12395-12399 (2013). https://doi.org/10.1039/c3ta1 $2691 \mathrm{a}$

224. L. Wang, Q. Yao, H. Bi, F. Huang, Q. Wang, L. Chen, Large thermoelectric power factor in polyaniline/graphene nanocomposite films prepared by solution-assistant dispersing method. J. Mater. Chem. A 2(29), 11107-11113 (2014). https ://doi.org/10.1039/c4ta01541j

225. Y. Du, K.F. Cai, S.Z. Shen, P.S. Casey, Preparation and characterization of graphene nanosheets/poly(3-hexylthiophene) thermoelectric composite materials. Synth. Met. 162(23), 2102-2106 (2012). https://doi.org/10.1016/j.synth met.2012.09.011

226. F. Li, K. Cai, S. Shen, S. Chen, Preparation and thermoelectric properties of reduced graphene oxide/PEDOT:PSS composite films. Synth. Met. 197, 58-61 (2014). https://doi. org/10.1016/j.synthmet.2014.08.014

227. Y. Jiang, Z. Wang, M. Shang, Z. Zhang, S. Zhang, Heat collection and supply of interconnected netlike graphene/ polyethyleneglycol composites for thermoelectric devices. Nanoscale 7(25), 10950-10953 (2015). https://doi. org/10.1039/c5nr02051d

228. G. Qiu, S. Huang, M. Segovia, P.K. Venuthurumilli, Y. Wang, W. Wu, X. Xu, P.D. Ye, Thermoelectric performance of 2D tellurium with accumulation contacts. Nano Lett. 19(3), 1955-1962 (2019). https://doi.org/10.1021/acs.nanol ett.8b05144

229. J.P. Heremans, M.S. Dresselhaus, L.E. Bell, D.T. Morelli, When thermoelectrics reached the nanoscale. Nat. Nanotechnol. 8(7), 471-473 (2013). https://doi.org/10.1038/nnano .2013 .129

230. J. Zeng, X. He, S.-J. Liang, E. Liu, Y. Sun et al., Experimental identification of critical condition for drastically enhancing thermoelectric power factor of two-dimensional layered materials. Nano Lett. 18(12), 7538-7545 (2018). https://doi. org/10.1021/acs.nanolett.8b03026

231. J.P. Small, K.M. Perez, P. Kim, Modulation of thermoelectric power of individual carbon nanotubes. Phys. Rev. Lett. 91(25), 256801 (2003). https://doi.org/10.1103/PhysRevLet t.91.256801

232. J. Lee, W. Lee, G. Wehmeyer, S. Dhuey, D.L. Olynick et al., Investigation of phonon coherence and backscattering using silicon nanomeshes. Nat. Commun. 8, 14054 (2017). https:// doi.org/10.1038/ncomms 14054

233. J.-K. Yu, S. Mitrovic, D. Tham, J. Varghese, J.R. Heath, Reduction of thermal conductivity in phononic nanomesh structures. Nat. Nanotechnol. 5(10), 718-721 (2010). https ://doi.org/10.1038/nnano.2010.149

234. M.T. Pettes, J. Maassen, I. Jo, M.S. Lundstrom, L. Shi, Effects of surface band bending and scattering on thermoelectric transport in suspended bismuth telluride nanoplates. Nano Lett. 13(11), 5316-5322 (2013). https://doi. org/10.1021/nl402828s

235. A.I. Boukai, Y. Bunimovich, J. Tahir-Kheli, J.-K. Yu, W.A. Goddard III, J.R. Heath, Silicon nanowires as efficient thermoelectric materials. Nature 451(7175), 168-171 (2008). https://doi.org/10.1038/nature06458

236. K. Hippalgaonkar, B. Huang, R. Chen, K. Sawyer, P. Ercius, A. Majumdar, Fabrication of microdevices with integrated nanowires for investigating low-dimensional phonon transport. Nano Lett. 10(11), 4341-4348 (2010). https://doi.org/10.1021/n1101671r

237. A.I. Hochbaum, R. Chen, R.D. Delgado, W. Liang, E.C. Garnett, M. Najarian, A. Majumdar, P. Yang, Enhanced thermoelectric performance of rough silicon nanowires. Nature 451(7175), 163-167 (2008). https://doi.org/10.1038/ nature 06381

238. J. Lim, H.-T. Wang, J. Tang, S.C. Andrews, H. So, J. Lee, D.H. Lee, T.P. Russell, P. Yang, Simultaneous thermoelectric property measurement and incoherent phonon transport in holey silicon. ACS Nano 10(1), 124-132 (2016). https:// doi.org/10.1021/acsnano.5b05385

239. J. Lee, J. Lim, P. Yang, Ballistic phonon transport in holey silicon. Nano Lett. 15(5), 3273-3279 (2015). https://doi. org/10.1021/acs.nanolett.5b00495

240. J. Tang, H.-T. Wang, D.H. Lee, M. Fardy, Z. Huo, T.P. Russell, P. Yang, Holey silicon as an efficient thermoelectric material. Nano Lett. 10(10), 4279-4283 (2010). https://doi. org/10.1021/nl102931z 
241. G. Zeng, J.M.O. Zide, W. Kim, J.E. Bowers, A.C. Gossard, Cross-plane seebeck coefficient of ErAs:InGaAs/InGaAlAs superlattices. J. Appl. Phys. 101(3), 035502 (2007). https:// doi.org/10.1063/1.2433751

242. B. Yang, W.L. Liu, J.L. Liu, K.L. Wang, G. Chen, Measurements of anisotropic thermoelectric properties in superlattices. Appl. Phys. Lett. 81(19), 3588-3590 (2002). https:// doi.org/10.1063/1.1515876

243. Z. Jia, C. Li, X. Li, J. Shi, Z. Liao, D. Yu, X. Wu, Thermoelectric signature of the chiral anomaly in $\mathrm{Cd}_{3} \mathrm{As}_{2}$. Nat. Commun. 7, 13013 (2016). https://doi.org/10.1038/ncomm s13013

244. J. Shen, Y. Jung, A.S. Disa, F.J. Walker, C.H. Ahn, J.J. Cha, Synthesis of SnTe nanoplates with 100 and 111 surfaces. Nano Lett. 14(7), 4183-4188 (2014). https://doi. org/10.1021/nl501953s

245. Y.M. Zuev, J.S. Lee, C. Galloy, H. Park, P. Kim, Diameter ependence of the transport properties of antimony telluride nanowires. Nano Lett. 10(8), 3037-3040 (2010). https:// doi.org/10.1021/nl101505q

246. H. Karamitaheri, M. Pourfath, R. Faez, H. Kosina, Geometrical effects on the thermoelectric properties of ballistic graphene antidot lattices. J. Appl. Phys. 110(5), 054506 (2011). https://doi.org/10.1063/1.3629990

247. J.Y. Kim, J.-H. Lee, J.C. Grossman, Thermal transport in functionalized graphene. ACS Nano 6(10), 9050-9057 (2012). https://doi.org/10.1021/nn3031595

248. N. Xiao, X. Dong, L. Song, D. Liu, Y. Tay et al., Enhanced thermopower of graphene films with oxygen plasma treatment. ACS Nano 5(4), 2749-2755 (2011). https://doi. org/10.1021/nn2001849

249. J. Oh, H. Yoo, J. Choi, J.Y. Kim, D.S. Lee et al., Significantly reduced thermal conductivity and enhanced thermoelectric properties of single- and bi-layer graphene nanomeshes with sub-10 nm neck-width. Nano Energy 35, 26-35 (2017). https ://doi.org/10.1016/j.nanoen.2017.03.019

250. T. Pei, L. Bao, R. Ma, S. Song, B. Ge et al., Epitaxy of ultrathin $\mathrm{SnSe}$ single crystals on polydimethylsiloxane: inplane electrical anisotropy and gate-tunable thermopower. Adv. Electron. Mater. 2(11), 1600292 (2016). https://doi. org/10.1002/aelm.201600292

251. M. Yoshida, T. Iizuka, Y. Saito, M. Onga, R. Suzuki, Y. Zhang, Y. Iwasa, S. Shimizu, Gate-optimized thermoelectric power factor in ultrathin $\mathrm{WSe}_{2}$ single crystals. Nano Lett. 16(3), 2061-2065 (2016). https://doi.org/10.1021/acs.nanol ett.6b00075

252. Z. Li, S.R. Bauers, N. Poudel, D. Hamann, X. Wang et al., Cross-plane seebeck coefficient measurement of misfit layered compounds $(\mathrm{SnSe})(\mathrm{n})\left(\mathrm{TiSe}_{2}\right)(\mathrm{n}),(\mathrm{n}=1,3,4,5)$. Nano Lett. 17(3), 1978-1986 (2017). https://doi.org/10.1021/acs. nanolett.6b05402

253. J. Chen, D.M. Hamann, D. Choi, N. Poudel, L. Shen, L. Shi, D.C. Johnson, S. Cronin, Enhanced cross-plane thermoelectric transport of rotationally disordered $\mathrm{SnSe}_{2}$ via Se-vapor annealing. Nano Lett. 18(11), 6876-6881 (2018). https://doi. org/10.1021/acs.nanolett.8b02744
254. Z.-Y. Juang, C.-C. Tseng, Y. Shi, W.-P. Hsieh, S. Ryuzaki et al., Graphene-Au nanoparticle based vertical heterostructures: a novel route towards high-ZT Thermoelectric devices. Nano Energy 38, 385-391 (2017). https://doi.org/10.1016/j. nanoen.2017.06.004

255. C.-C. Chen, Z. Li, L. Shi, S.B. Cronin, Thermoelectric transport across graphene/hexagonal boron nitride/graphene heterostructures. Nano Res. 8(2), 666-672 (2014). https://doi. org/10.1007/s12274-014-0550-8

256. J.T. Robinson, S.M. Tabakman, Y. Liang, H. Wang, H.S. Casalongue, V. Daniel, H. Dai, Ultrasmall reduced graphene oxide with high near-infrared absorbance for photothermal therapy. J. Am. Chem. Soc. 133(17), 6825-6831 (2011). https ://doi.org/10.1021/ja2010175

257. J. Shao, H. Xie, H. Huang, Z. Li, Z. Sun et al., Biodegradable black phosphorus-based nanospheres for in vivo photothermal cancer therapy. Nat. Commun. 7, 12967 (2016). https:// doi.org/10.1038/ncomms12967

258. K. Yang, S. Zhang, G. Zhang, X. Sun, S.T. Lee, Z. Liu, Graphene in mice: ultrahigh in vivo tumor uptake and efficient photothermal therapy. Nano Lett. 10(9), 3318-3323 (2010). https://doi.org/10.1021/nl100996u

259. Z. Sun, H. Xie, S. Tang, X.-F. Yu, Z. Guo et al., Ultrasmall black phosphorus quantum dots: synthesis and use as photothermal agents. Angew. Chem. Int. Ed. 54(39), 11526-11530 (2015). https://doi.org/10.1002/anie.201506154

260. N.M. Gabor, J.C.W. Song, Q. Ma, N.L. Nair, T. Taychatanapat et al., Hot carrier-assisted intrinsic photoresponse in graphene. Science 334(6056), 648-652 (2011). https://doi. org/10.1126/science. 1211384

261. Y. Yan, Z.-M. Liao, X. Ke, G. Van Tendeloo, Q. Wang et al., Topological surface state enhanced photothermoelectric effect in $\mathrm{Bi}_{2} \mathrm{Se}_{3}$ nanoribbons. Nano Lett. 14(8), 4389-4394 (2014). https://doi.org/10.1021/nl501276e

262. D.J. Groenendijk, M. Buscema, G.A. Steele, S.M. de Vasconcellos, R. Bratschitsch, H.S.J. van der Zant, A. Castellanos-gomez, Photovoltaic and photothermoelectric effect in a double-gated $\mathrm{WSe}_{2}$ device. Nano Lett. 14(10), 5846-5852 (2014). https://doi.org/10.1021/nl502741k

263. B.C. St-Antoine, D. Menard, R. Martel, Position sensitive photothermoelectric effect in suspended single-walled carbon nanotube films. Nano Lett. 9(10), 3503-3508 (2009). https:// doi.org/10.1021/n1901696j

264. Y. Zhang, H. Li, L. Wang, H. Wang, X. Xie, S.-L. Zhang, R. Liu, Z.-J. Qiu, Photothermoelectric and photovoltaic effects both present in $\mathrm{MoS}_{2}$. Sci. Rep. 5, 7938 (2015). https://doi. org/10.1038/srep07938

265. D. Kraemer, B. Poudel, H.-P. Feng, J.C. Caylor, B. Yu et al., High-performance flat-panel solar thermoelectric generators with high thermal concentration. Nat. Mater. 10(7), 532-538 (2011). https://doi.org/10.1038/nmat3013

266. X. Cai, A.B. Sushkov, R.J. Suess, M.M. Jadidi, G.S. Jenkins et al., Sensitive room-temperature terahertz detection via the photothermoelectric effect in graphene. Nat. Nanotechnol. 9(10), 814-819 (2014). https://doi.org/10.1038/nnano .2014 .182 
267. J.E. Muench, A. Ruocco, M.A. Giambra, V. Miseikis, D. Zhang et al., Waveguide-integrated, plasmonic enhanced graphene photodetectors. Nano Lett. 19(11), 7632-7644 (2019). https://doi.org/10.1021/acs.nanolett.9b02238
268. E. Leong, R.J. Suess, A.B. Sushkov, H.D. Drew, T.E. Murphy, M. Mittendorff, Terahertz photoresponse of black phosphorus. Opt. Express 25(11), 12666-12674 (2017). https://doi. org/10.1364/OE.25.012666 